\title{
HYPERBOLIC AND PARABOLIC UNIMODULAR RANDOM MAPS
}

\author{
OMER ANGEL TOM HUTCHCROFT ASAF NACHMIAS GOURAB RAY
}

\begin{abstract}
We show that for infinite planar unimodular random rooted maps, many global geometric and probabilistic properties are equivalent, and are determined by a natural, local notion of average curvature. This dichotomy includes properties relating to amenability, conformal geometry, random walks, uniform and minimal spanning forests, and Bernoulli bond percolation. We also prove that every simply connected unimodular random rooted map is sofic, that is, a Benjamini-Schramm limit of finite maps.
\end{abstract}

\section{Introduction}

In the classical theory of Riemann surfaces, the Uniformization Theorem states that every simply connected, non-compact Riemann surface is conformally equivalent to either the plane or the disc, which are inequivalent to each other by Liouville's theorem. The dichotomy provided by this theorem manifests itself in several different ways, relating to analytic, geometric and probabilistic properties of surfaces. In particular, if $S$ is a simply connected, non-compact Riemann surface, then either

$S$ is parabolic: it is conformally equivalent to the plane, admits a compatible Riemannian metric of constant curvature 0 , does not admit non-constant bounded harmonic functions, and is recurrent for Brownian motion,

or else

$S$ is hyperbolic: it is conformally equivalent to the disc, admits a compatible Riemannian metric of constant curvature -1 , admits non-constant bounded harmonic functions, and is transient for Brownian motion.

In the 1990's, a discrete counterpart to this dichotomy began to develop in the setting of bounded degree planar graphs $[8,44,43,17,18]$. A major milestone in this theory was the seminal work of He and Schramm [44, 43], who studied circle packings of infinite triangulations of the plane. They proved that every infinite triangulation of the plane can be circle packed in either the unit disc or in the plane, but not both. A triangulation is called CP hyperbolic or CP parabolic accordingly. He and Schramm also connected the circle packing type to isoperimetric and probabilistic properties of the triangulation, showing in particular that, in the bounded degree case, CP parabolicity is equivalent to the recurrence of simple random walk. Later, Benjamini and Schramm $[17,18]$ provided an analytic aspect to this dichotomy, showing that every bounded degree, infinite planar graph admits non-constant bounded harmonic functions if and only if it is transient for simple random walk, and that in this case the graph also admits non-constant bounded harmonic functions of finite Dirichlet energy. Most of this theory fails without the assumption of bounded degrees, as one can easily construct pathological counter-examples to the theorems above.

The goal of this paper is to develop a similar theory for unimodular random rooted maps, without the assumption of bounded degree. In our earlier work [4], we studied circle packings

Date: 2018-03-22. 
of, and random walks on, random plane triangulations of unbounded degree. In this paper, we study many further properties of unimodular random planar maps, which we do not assume to be triangulations. Our results also have consequences for unimodular random maps that are not planar, which we develop in Section 7. Our main result may be stated informally as follows; see Theorem 1 for a complete and precise statement.

Theorem (The Dichotomy Theorem). Every infinite, planar, unimodular random rooted planar map is either hyperbolic or parabolic. The map is hyperbolic if and only if its average curvature is negative and is parabolic if and only if its average curvature is zero. The type of a unimodular random rooted map determines many of its properties.

The many properties we show to be determined by the type of the map are far-reaching, relating to aspects of the map including amenability, random walks, harmonic functions, spanning forests, Bernoulli bond percolation, and the conformal type of associated Riemann surfaces. The seeds of such a dichotomy were already apparent in [4], in which we proved that a unimodular random rooted plane triangulation is CP parabolic almost surely if and only if the expected degree of the root is six (which is equivalent to the average curvature being zero), if and only if the triangulation is invariantly amenable - a notion of amenability due to Aldous and Lyons [2] that is particularly suitable to unimodular random rooted graphs. A notable property that is not a part of Theorem 1 is recurrence of the random walk: while every hyperbolic unimodular random rooted map is transient, not every parabolic unimodular random rooted map is recurrent. (Theorem 1 can be combined with the work of Gurel-Gurevich and the third author [38] to deduce that a parabolic unimodular random planar map is recurrent under the additional assumption that the degree of the root has an exponential tail.)

A map is a proper (i.e., locally finite) embedding of a graph into an oriented surface viewed up to orientation preserving homeomorphisms of the surface. (Other definitions extend to non-orientable maps, but we shall not be concerned with those here.) A rooted map is a map together with a distinguished root vertex. The map is called planar if the surface is homeomorphic to an open subset of the sphere, and is simply connected if the surface is homeomorphic to the sphere or the plane. (In particular, every simply connected map is planar.) A random rooted map is said to be unimodular if it satisfies the mass-transport principle, which can be interpreted as meaning that 'every vertex of the map is equally likely to be the root'. See Section 2 for precise definitions of each of these terms.

The curvature of a map is a local geometric property, closely related to the Gaussian curvature of manifolds that may be constructed from the map; see Section 4 for a precise definition. For one natural manifold constructed from the map by gluing together regular polygons, the curvature at each vertex $v$ is

$$
\kappa(v)=2 \pi-\sum_{f \perp v} \frac{\operatorname{deg}(f)-2}{\operatorname{deg}(f)} \pi,
$$

where the sum is taken over faces of the map incident to $v$, and a face is counted with multiplicity if more than one of the corners of the face are located at $v$. We can define the average curvature of $(M, \rho)$ to be the expectation $\mathbb{E}[\kappa(\rho)]$. Theorem 4.10 states that the average curvature is a canonical quantity associated to the random map, in the sense that any unimodular way of associating a manifold to the map will result in the same average curvature. Observe that the average curvature of a unimodular random triangulation is equal 
to $(6-\mathbb{E}[\operatorname{deg}(\rho)]) \pi / 3$, so that a unimodular triangulation has expected degree greater than six if and only if it has negative average curvature. This relates the dichotomy described in [4] to that of Theorem 1.

Classical examples of unimodular random maps are provided by Voronoi diagrams of stationary point processes [16] and (slightly modified) Galton-Watson trees [2, Example 1.1], as well as lattices in the Euclidean and hyperbolic planes, and arbitrary local limits of finite maps. Many local modifications of maps, such as taking Bernoulli percolation or uniform or minimal spanning trees, preserve unimodularity, giving rise to many additional examples. Unimodular random maps, most notably the uniform infinite planar triangulation (UIPT) [7] and quadrangulation (UIPQ) [55, 29], have also been studied in the context of 2-dimensional quantum gravity; see the survey [35] and references therein. More recently, hyperbolic variants of the UIPT have been constructed [5, 28]. Many of these examples do not have uniformly bounded degrees, so that the deterministic theory is not applicable to them.

\subsection{The Dichotomy Theorem}

Since many of the notions tied together in the following theorem are well known, we first state the theorem, and defer definitions to individual sections dealing with each of the properties.

Theorem 1 (The Dichotomy Theorem). Let $(M, \rho)$ be an infinite, ergodic, unimodular random rooted planar map and suppose that $\mathbb{E}[\operatorname{deg}(\rho)]<\infty$. Then the average curvature of $(M, \rho)$ is non-positive and the following are equivalent:

(1) $(M, \rho)$ has average curvature zero.

(2) $(M, \rho)$ is invariantly amenable.

(3) Every bounded degree subgraph of $M$ is amenable almost surely.

(4) Every subtree of $M$ is amenable almost surely.

(5) Every bounded degree subgraph of $M$ is recurrent almost surely.

(6) Every subtree of $M$ is recurrent almost surely.

(7) $(M, \rho)$ is a Benjamini-Schramm limit of finite planar maps.

(8) $(M, \rho)$ is a Benjamini-Schramm limit of a sequence $\left\langle M_{n}\right\rangle_{n \geq 0}$ of finite maps such that

$$
\frac{\operatorname{genus}\left(M_{n}\right)}{\#\left\{\text { vertices of } M_{n}\right\}} \underset{n \rightarrow \infty}{\longrightarrow} 0 \text {. }
$$

(9) The Riemann surface associated to $M$ is conformally equivalent to either the plane $\mathbb{C}$ or the cylinder $\mathbb{C} / \mathbb{Z}$ almost surely.

(10) $M$ does not admit any non-constant bounded harmonic functions almost surely.

(11) $M$ does not admit any non-constant harmonic functions of finite Dirichlet energy almost surely.

(12) The laws of the free and wired uniform spanning forests of $M$ coincide almost surely.

(13) The wired uniform spanning forest of $M$ is connected almost surely.

(14) Two independent random walks on $M$ intersect infinitely often almost surely.

(15) The laws of the free and wired minimal spanning forests of $M$ coincide almost surely.

(16) Bernoulli(p) bond percolation on $M$ has at most one infinite connected component for every $p \in[0,1]$ almost surely (in particular, $p_{c}=p_{u}$ ).

(17) $M$ is vertex extremal length parabolic almost surely. 


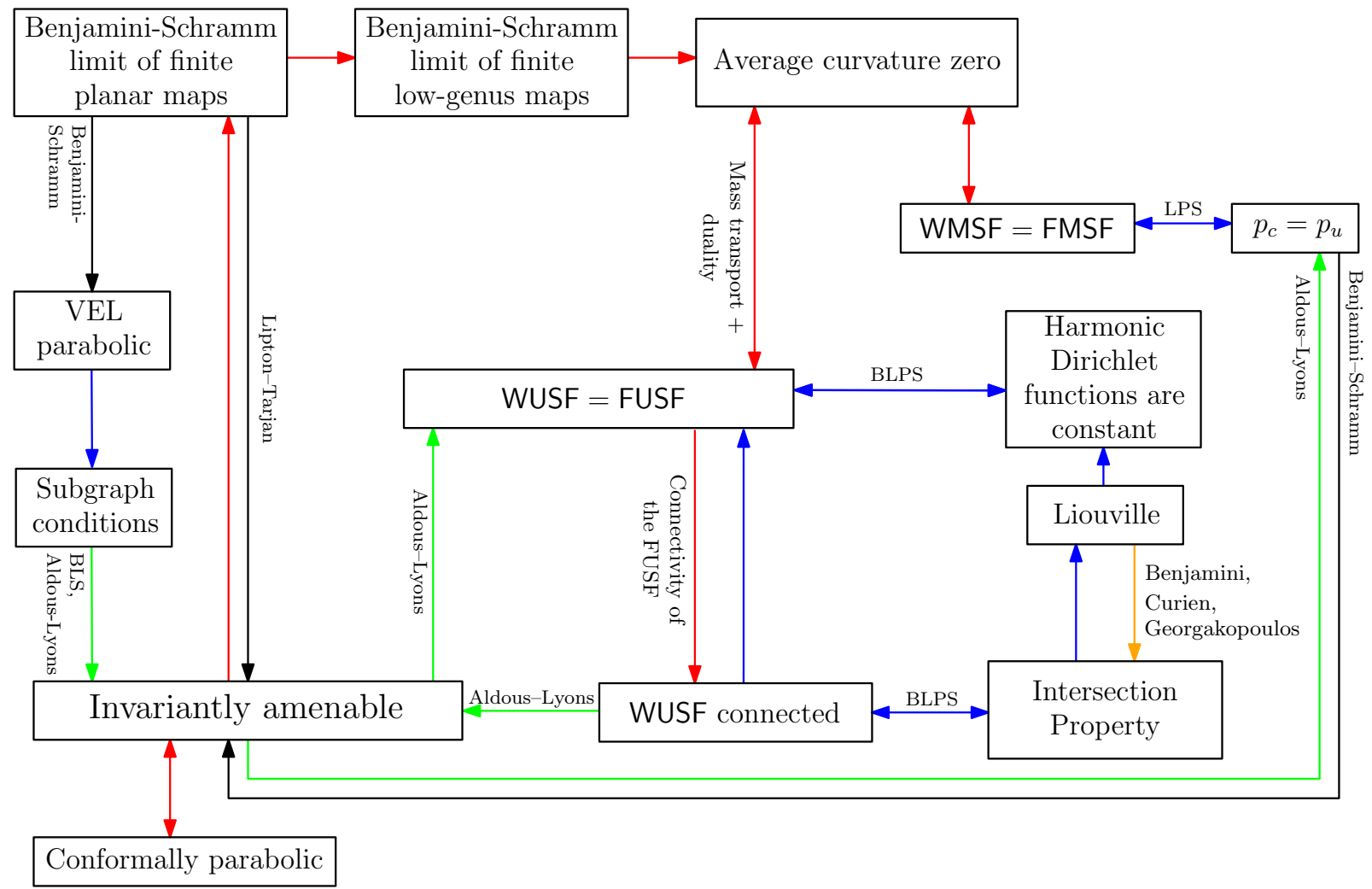

Figure 1. The logical structure for the proof of Theorem 1 in the simply connected case. Implications new to this paper are in red. Blue implications hold for arbitrary graphs; the orange implication holds for arbitrary planar graphs, and green implications hold for unimodular random rooted graphs even without planarity. A few implications between items that are known but not used in the proof are omitted.

In light of this theorem, we call a unimodular random rooted map $(M, \rho)$ with $\mathbb{E}[\operatorname{deg}(\rho)]<$ $\infty$ parabolic if its average curvature is zero (and, in the planar case, clauses (1)-(17) all hold), and hyperbolic if its average curvature is negative (and, in the planar case, the clauses all fail).

As one might guess from the structure of Theorem 1, the proof consists of many separate arguments for the different implications. Some of the implications are already present in the literature, and part of this paper is spent surveying the earlier works that form the individual implications between the long list of equivalent items in Theorem 1. For the sake of completeness we also include proofs of several standard technical results from the ergodic theory literature using probabilisitic terminology.

Some of the implications in Theorem 1 hold in any graph. For example, (10) implies (11) for any graph, and (14) and (13) are always equivalent (see [13]). Other implications hold for any planar graph. For example (10) is equivalent to (14), see [10]. We do not provide a comprehensive list of the assumptions needed for each implication, but some of this information is encoded in Figure 1. 


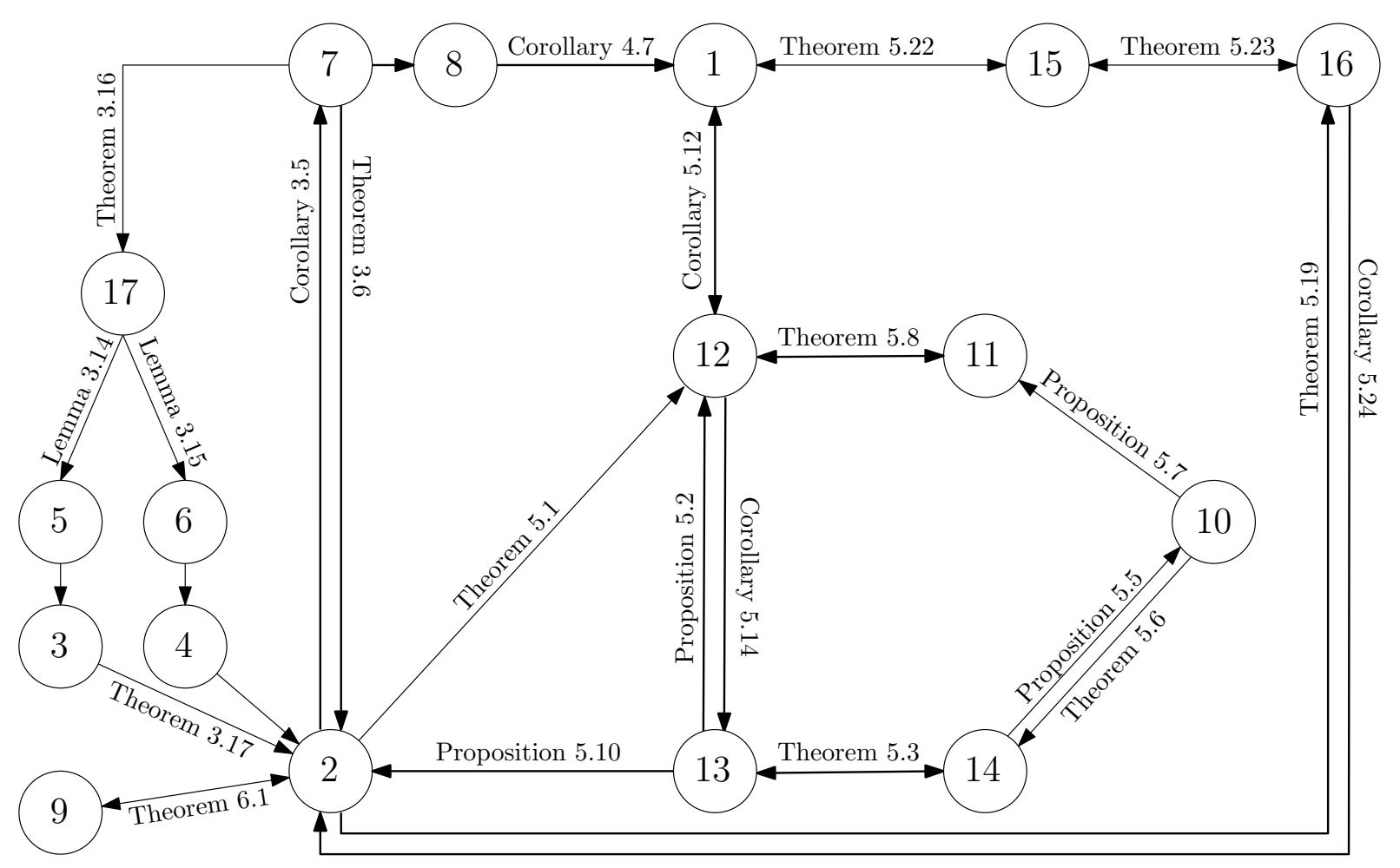

Figure 2. The numbers of the theorems, propositions, lemmas and corrolaries forming the individual implications used to prove Theorem 1 in the simply connected case. Unlabelled implications are trivial.

Most of the paper is dedicated to proving the theorem under the additional assumption that $M$ is simply connected; the multiply-connected case is easier and is handled separately in Section 7. The logical structure of the proof in the simply connected case is summarized in Figures 1 and 2. Figure 1 also shows which implications were already known and which are proved in the present paper.

Let us note that there are several natural ways of turning a planar map into a triangulation, which allow some of the implications between items in Theorem 1 to be reduced to the case of triangulations, in which some of the implications were already treated in [4]. However, many of the properties listed in Theorem 1 are not invariant to taking subgraphs, and most of the properties were not treated in [4]. Thus, we cannot rely on the methods of our previous paper [4].

\subsection{Unimodular planar maps are sofic}

Let $\left\langle G_{n}\right\rangle_{n \geq 0}$ be a sequence of (possibly random) finite graphs. We say that a random rooted graph $(G, \rho)$ is the Benjamini-Schramm limit of the sequence $\left\langle G_{n}\right\rangle_{n \geq 0}$ if the random rooted graphs $\left(G_{n}, \rho_{n}\right)$ converge in distribution to $(G, \rho)$ with respect to the local topology on rooted graphs (see Section 2.2), where $\rho_{n}$ is a uniform vertex of $G_{n}$. Benjamini-Schramm limits of finite maps, marked graphs and marked maps are defined similarly, except that the local topology takes into account the additional structure. When $G_{n}$ is a uniformly chosen map of size $n$, this construction gives rise to the aforementioned UIPT and UIPQ. 
It is easy to see that every (possibly random) finite graph with conditionally uniform root is unimodular. Moreover, unimodularity is preserved under distributional limits in the local topology. It follows that every Benjamini-Schramm limit of finite random graphs is unimodular. A random rooted graph that can be obtained in this way is called sofic. It is a major open problem to determine whether the converse holds, that is, whether every unimodular random rooted graph is sofic [2, Section 10]. The next theorem answers this question positively for simply connected unimodular random maps.

Theorem 2. Every simply connected unimodular random rooted map is sofic.

The proof of Theorem 2 relies on the corresponding result for trees, which is due to Bowen [23], Elek [32], and Benjamini, Lyons and Schramm [15]. As was observed by Elek and Lippner [33], it follows that treeable unimodular graphs (that is, unimodular graphs that exhibit an invariant spanning tree) are sofic, and so the key new step is proving the connectivity of the free uniform spanning forest.

Note that the finite maps converging to a given infinite unimodular random rooted map $(M, \rho)$ need not be planar. Indeed, Theorem 1 characterises the Benjamini-Schramm limits of finite planar maps exactly as the parabolic unimodular random rooted maps. Moreover, if $\left\langle M_{n}\right\rangle_{n \geq 0}$ is a sequence of finite maps converging to an infinite hyperbolic unimodular random rooted map, then the approximating maps $M_{n}$ must have genus comparable to their number of vertices as $n$ tends to infinity.

\section{Unimodular Maps}

\subsection{Maps}

We provide here a brief background to the concept of maps, and refer the reader to [56, Chapter 1.3] for a comprehensive treatment. Let $G=(V, E)$ be a connected graph, which may contain self-loops and multiple edges. An embedding of a graph in a surface $S$ is a drawing of the graph in the surface with non-crossing edges. Given an embedding, the connected components of the complement of the image of $G$ are called faces. An embedding is said to be proper if the following conditions hold:

(1) it is locally finite (every compact set in $S$ intersects finitely many edges),

(2) every face is homeomorphic to an open disc, and

(3) for every face $f$, if we consider the oriented edges of $G$ that have their right hand side incident to $f$, and consider the permutation that maps each such oriented edge to the oriented edge following it in the clockwise order around the face, then this permutation has a single orbit.

For example, the complete graph on three vertices can be properly embedded in the sphere but not in the plane. If $S$ is simply connected or compact, then any embedding that satisfies (1) and (2) must also satisfy (3). For this reason, the condition (3) is not included in many references that deal primarily with finite maps. An example of an embedding that satisfies (1) and (2) but not (3) is given by drawing $\mathbb{Z}$ along a straight line in an infinite cylinder.

We define a (locally finite) map $M$ to be a connected, locally finite graph $G$ together with an equivalence class of proper embeddings of $G$ into oriented surfaces, where two embeddings are equivalent if there is an orientation preserving homeomorphism between the two surfaces that sends one embedding to the other. If $M$ is a map with underlying graph $G$, we refer to 
any proper embedding of $G$ that falls into the equivalence class of embeddings corresponding to $M$ as an embedding of $M$. A map is said to be planar if it is embedded into a surface homeomorphic to an open subset of the sphere, and is said to be simply connected if it is embedded into a simply connected surface (which is necessarily homeomorphic to either the sphere or the plane).

Let $M$ be a map with underlying graph $G$ and let $z$ be a proper embedding of $M$ into a surface $S$. If every face of $M$ has finite degree, the dual map of $M$, denoted $M^{\dagger}$ is defined as follows. The underlying graph of $M^{\dagger}$, denoted $G^{\dagger}$, has the faces of $M$ as vertices, and has an edge drawn between two faces of $M$ for each edge in $M$ that is incident to both of the faces. We define an embedding $z^{\dagger}$ of $G^{\dagger}$ into $S$ by placing each vertex of $G^{\dagger}$ in the interior of the corresponding face of $M$ and each edge of $G^{\dagger}$ so that it crosses the corresponding edge of $G$ but no others. We define $M^{\dagger}$ to be the map with underlying graph $G$ represented by the pair $\left(S, z^{\dagger}\right)$ : Although the embedding $z^{\dagger}$ is not uniquely defined, every choice of $z^{\dagger}$ defines the same map. The construction gives a canonical bijection between edges of $G$ and edges of $G^{\dagger}$. We write $e^{\dagger}$ for the edge of $G^{\dagger}$ corresponding to $e$. If $e$ is an oriented edge, we let $e^{\dagger}$ be oriented so that it crosses $e$ from right to left as viewed from the orientation of $e$.

Despite their topological definitions, maps and their duals can in fact be defined entirely combinatorially. Given any graph, we consider each edge as two oriented edges in opposite directions. We write $E^{\rightarrow}=E^{\rightarrow}(G)$ for the set of oriented edges of a graph. For each directed edge $e$, we have a head $e^{+}$and tail $e^{-}$, and write $-e$ for the reversal of $e$. Given a map $M$ and a vertex $v$ of $M$, let $\sigma_{v}=\sigma_{v}(M)$ be the cyclic permutation of the set $\left\{e \in E^{\rightarrow}: e^{-}=v\right\}$ of oriented edges emanating from $v$ corresponding to counter-clockwise rotation in $S$. This procedure defines a bijection between maps and graphs labelled by cyclic permutations.

Theorem 2.1 ([56]). Given a connected, locally finite graph $G$ and a collection of cyclic permutations $\sigma_{v}$ of the sets $\left\{e \in E^{\rightarrow}: e^{-}=v\right\}$, there exists a unique map $M$ with underlying graph $G$ such that $\sigma(M)=\sigma$.

In light of Theorem 2.1, we formally identify a map $M$ with the pair $(G, \sigma)$. Given such a combinatorial specification of a map $M$ as a pair $(G, \sigma)$, we may form an embedding of the map into a surface $S(M)$ by gluing topological discs according to the combinatorics of the map (see Figure 3).

Note that we can write $\sigma(e)$ for $\sigma_{v}(e)$, since necessarily $v=e^{-}$. Thus $\sigma$ is a permutation on the set of directed edges of $M$. Formally, a corner in the map at a vertex $v$ is an ordered pair of directed edges $(e, \sigma(e))$, with $e^{-}=v$. Of course, a corner is determined by the directed edge $e$. Just as vertices of $M$ are orbits of $\sigma$, the faces of a map $M=(G, \sigma)$ can be defined abstractly as orbits of the permutation $\sigma^{\dagger}: E^{\rightarrow} \rightarrow E^{\rightarrow}$ defined by $\sigma^{\dagger}(e)=\sigma^{-1}(-e)$ for each $e \in E^{\rightarrow}$. The dual $e^{\dagger}$ of a directed edge $e$ is defined to have the orbit of $e$ as its tail and the orbit of $-e$ as its head, so that we again have a bijection between directed edges of $M$ and their duals. Using this bijection, we can consider $\sigma^{\dagger}$ to acts on dual edges, and the dual map $M^{\dagger}$ is then constructed abstractly as $M^{\dagger}=\left(G^{\dagger}, \sigma^{\dagger}\right)$. We define maps with infinite degree vertices, and duals of maps with infinite degree faces, directly through this abstract formalism.

If $e$ is an oriented edge in a map, we write $e^{\ell}$ for the face of $M$ to the left of $e$ and $e^{r}$ for the face of $M$ to the right of $e$, so that $e^{\ell}=\left(e^{\dagger}\right)^{-}$and $e^{r}=\left(e^{\dagger}\right)^{+}$. Given a map $M$, we write $f \perp v$ if the face $f$ is incident to the vertex $v$, that is, if there exists an oriented edge $e$ of $M$ such that $e^{-}=v$ and $e^{\ell}=f$. When writing a sum of the form $\sum_{f \perp v}$, we use the convention that a face $f$ is counted with multiplicity according to the number of oriented edges $e$ of $M$ 

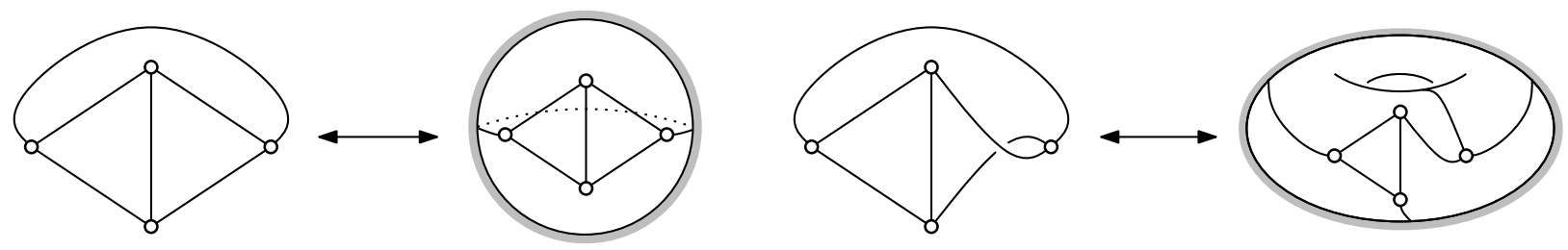

Figure 3. Different maps with the same underlying graph. The two maps both have $K_{4}$ as their underlying graph, but the left is a sphere map while the right is a torus map. Both maps are represented both abstractly as a graph together with a cyclic permutation of the edges emanating from each vertex (left) and as a graph embedded in a surface (right).

such that $e^{-}=v$ and $e^{\ell}=f$. Similarly, when writing a sum of the form $\sum_{u \sim v}$, we count each vertex $u$ with multiplicity according to the number of oriented edges $e$ of $M$ such that $e^{-}=v$ and $e^{+}=u$. The degree of a face is defined to be the number of oriented edges with $e^{r}=f$, i.e., the degree of the face in the dual.

\subsection{Unimodularity and the mass transport principle}

As noted, a rooted graph $(G, \rho)$ is a connected, locally finite (multi)graph $G=(V, E)$ together with a distinguished vertex $\rho$, called the root. A graph isomorphism $\phi: G \rightarrow G^{\prime}$ is an isomorphism of rooted graphs is a graph isomorphism that maps the root to the root.

The local topology (see [20]) is the topology on the set $\mathcal{G}$. of isomorphism classes of rooted graphs induced by the metric

$$
d_{\mathrm{loc}}\left((G, \rho),\left(G^{\prime}, \rho^{\prime}\right)\right)=e^{-R},
$$

where

$$
R=R\left((G, \rho),\left(G^{\prime}, \rho^{\prime}\right)\right)=\sup \left\{R \geq 0: B_{R}(G, \rho) \cong B_{R}\left(G^{\prime}, \rho^{\prime}\right)\right\},
$$

i.e., the maximal radius such that the balls $B_{R}(G, \rho)$ and $B_{R}\left(G^{\prime}, \rho^{\prime}\right)$ are isomorphic as rooted graphs.

A random rooted graph is a random variable taking values in the space $\mathcal{G} \bullet$ endowed with the local topology. Similarly, a doubly-rooted graph is a graph together with an ordered pair of distinguished (not necessarily distinct) vertices. Denote the space of isomorphism classes of doubly-rooted graphs equipped with this topology by $\mathcal{G}$...

The spaces of rooted and doubly-rooted maps are defined similarly and are denoted $\mathcal{M}$. and $\mathcal{M}_{\text {.. }}$ respectively. For this, an isomorphism $\phi$ of rooted maps is preserves the roots and the map structure, so that $\phi \circ \sigma=\sigma^{\prime} \circ \phi$ for vertices inside the balls.

A further generalisation of these spaces will also be useful. A marked graph (referred to by Aldous and Lyons [2] as a network) is defined to be a locally finite, connected graph together with a function $m: E \cup V \rightarrow \mathbb{X}$ assigning each vertex and edge of $G$ a mark in some Polish space $\mathbb{X}$, referred to as the mark space. The local topology on the set of isomorphism classes of rooted graphs with marks in $\mathbb{X}$ is the topology induced by the metric

$$
d_{\mathrm{loc}}\left((G, \rho, m),\left(G^{\prime}, \rho^{\prime}, m^{\prime}\right)\right)=e^{-R}
$$

where $R\left((G, \rho, m),\left(G^{\prime}, \rho^{\prime}, m^{\prime}\right)\right)$ is the largest $R$ such that there exists an isomorphism of rooted graphs $\phi:\left(B_{G}(\rho, b), m, \rho\right) \rightarrow\left(B_{G^{\prime}}\left(\rho^{\prime}, b\right), \rho^{\prime}\right)$ such that $d_{\mathbb{X}}\left(m^{\prime}(\phi(x)), m(x)\right) \leq 1 / R$ for every vertex or edge $x$ of $B_{G}(\rho, n)$, and $d_{\mathbb{X}}$ is a metric compatible with the topology of $\mathbb{X}$. 
The space of isomorphism classes of rooted marked graphs with marks in $\mathbb{X}$ is denoted $\mathcal{G}_{\bullet}^{\mathbb{X}}$. The space of rooted marked maps is defined similarly.

It is possible to consider rooted maps and rooted marked maps as rooted marked graphs by encoding the permutations $\sigma_{v}$ in the marks - in particular, this means that any statement that holds for all unimodular random rooted marked graphs also holds for all unimodular random rooted marked maps. See [2, Example 9.6].

A mass transport is a Borel function $f: \mathcal{G}_{\bullet \bullet} \rightarrow[0, \infty]$. A random rooted graph $(G, \rho)$ is said to be unimodular if it satisfies the Mass Transport Principle: for every mass transport $f$,

$$
\mathbb{E}\left[\sum_{v \in V} f(G, \rho, v)\right]=\mathbb{E}\left[\sum_{u \in V} f(G, u, \rho)\right] .
$$

That is,

\section{Expected mass out equals expected mass in.}

Unimodularity of random rooted maps, marked graphs and marked maps are defined similarly. A probability measure $\mathbb{P}$ on $\mathcal{G}_{\bullet}$ is said to be unimodular if a random rooted map with law $\mathbb{P}$ is unimodular. Unimodular probability measures on rooted maps, marked graphs and marked maps are defined similarly.

It will also be convenient to work with signed mass transports. If a measurable $f: \mathcal{G}_{\bullet \bullet} \rightarrow \mathbb{R}$ satsifies

$$
\mathbb{E}\left[\sum_{v \in V}|f(G, \rho, v)|\right]<\infty,
$$

then the conclusion of the mass transport principle holds for $f$. This follows easily from the usual mass transport principle for non-negative $f$.

The mass transport principle was first introduced by Häggström [40] to study dependent percolation on Cayley graphs. The formulation of the mass transport principle presented here was suggested by Benjamini and Schramm [20] and developed systematically by Aldous and Lyons [2]. The unimodular probability measures on $\mathcal{G}$ • form a weakly closed, convex subset of the space of probability measures on $\mathcal{G}_{\bullet}$, so that weak limits of unimodular random graphs are unimodular. In particular, a weak limit of finite graphs with uniformly chosen roots is unimodular: such a limit of finite graphs is referred to as a Benjamini-Schramm limit. It is a major open problem to determine whether all unimodular random rooted graphs arise as Benjamini-Schramm limits of finite graphs [2, §10].

We will make frequent use of the fact that unimodularity is stable under most reasonable ways of modifying a graph locally without changing its vertex set. The following lemma is a formalization of this fact.

Lemma 2.2. Let $\mathbb{X}_{1}$ and $\mathbb{X}_{2}$ be polish spaces. Let $(G, \rho, m)$ be a unimodular random rooted $\mathbb{X}_{1}$-marked graph, and suppose that $\left(G^{\prime}, \rho, m^{\prime}\right)$ is a random rooted marked graph with the same vertex set as $G$, such that, for every pair of vertices $u, v$ in $G$, the conditional distribution of $\left(G^{\prime}, u, v, m^{\prime}\right)$ given $(G, \rho, m)$ coincides a.s. with some measurable function of the isomorphism class of $(G, u, v, m)$. Then $\left(G^{\prime}, \rho, m^{\prime}\right)$ is unimodular.

Proof. Let $f: \mathcal{G}_{\bullet_{\bullet}}^{\mathbb{X}_{2}} \rightarrow[0, \infty]$ be a mass transport. For each pair of vertices $u, v$ in $G$, the conditional expectation

$$
F(G, u, v, m)=\mathbb{E}\left[f\left(G^{\prime}, u, v, m^{\prime}\right) \mid(G, \rho, m)\right]
$$


coincides a.s. with a measurable function of $(G, u, v, m)$, and so is itself a mass transport. Since $(G, \rho, m)$ is unimodular we deduce that

$$
\begin{aligned}
\mathbb{E} \sum_{v} f\left(G^{\prime}, \rho, v, m^{\prime}\right)=\mathbb{E} \sum_{v} F(G, \rho, v, & \\
& =\mathbb{E} \sum_{v} F(G, v, \rho, m)=\mathbb{E} \sum_{u} f\left(G^{\prime}, u, \rho, m^{\prime}\right),
\end{aligned}
$$

verifying that $\left(G^{\prime}, \rho, m^{\prime}\right)$ satisfies the mass transport principle.

Later in the paper, we will often claim that various random rooted graphs obtained from unimodular random rooted graphs in various ways are unimodular. These claims can always be justified either as a direct consequence of Lemma 2.2, or otherwise by applying the mass transport principle to the conditional expectation as in eq. (2.1).

\subsection{Reversibility}

Recall that the simple random walk on a locally finite graph $G=(V, E)$ with no isolated vertices is the Markov process $\left\langle X_{n}\right\rangle_{n \geq 0}$ on the state space $V$ with transition probabilities $p(u, v)$ defined to be the fraction of edges emanating from $u$ that end in $v$. A probability measure $\mathbb{P}$ on $\mathcal{G}_{\bullet}$ is said to be stationary if, when $(G, \rho)$ is a random rooted graph with law $\mathbb{P}$ and $\left\langle X_{n}\right\rangle_{n \geq 0}$ is a simple random walk on $G$ started at the root, $(G, \rho)$ and $\left(G, X_{n}\right)$ have the same distribution for all $n$ (in particular we require that every vertex of $(G, \rho)$ has at least one edge a.s., since otherwise the random walk is not defined).

The measure $\mathbb{P}$ is said to be reversible if furthermore

$$
\left(G, \rho, X_{n}\right) \stackrel{d}{=}\left(G, X_{n}, \rho\right)
$$

for all $n$. A random rooted graph is said to be reversible if its law is reversible. Reversible random rooted maps, marked graphs and marked maps are defined similarly. Every reversible random rooted graph is clearly stationary, but the converse need not hold in general [12, Examples 3.1 and 3.2].

Let $\mathcal{G}_{\leftrightarrow}$ be the space of isomorphism classes of connected, locally finite graphs equipped with a distinguished bi-infinite path, which we endow with a natural variant of the local topology. If $(G, \rho)$ is a reversible random rooted graph and $\left\langle X_{n}\right\rangle_{n \geq 0}$ and $\left\langle X_{-n}\right\rangle_{n \geq 0}$ are independent simple random walks started from $\rho$, then the sequence

$$
\left\langle\left(G,\left\langle X_{n+k}\right\rangle_{n \in \mathbb{Z}}\right)\right\rangle_{k \in \mathbb{Z}}
$$

of $\mathcal{G}_{\leftrightarrow}$-valued random variables is stationary.

The following correspondence between unimodular and reversible random rooted graphs is implicit in [2] and was proven explcitly in [9]. Similar correspondences also hold between unimodular and reversible random rooted maps, marked graphs and marked maps.

Proposition $2.3([2,9])$. Let $\mathbb{P}$ be a unimodular probability measure on $\mathcal{G} \bullet$ with $\mathbb{E}[\operatorname{deg}(\rho)]<\infty$ and let $\mathbb{P}_{\text {rev }}$ denote the $\operatorname{deg}(\rho)$-biasing of $\mathbb{P}$, defined by

$$
\mathbb{P}_{\mathrm{rev}}((G, \rho) \in \mathscr{A}):=\frac{\mathbb{E}[\operatorname{deg}(\rho) \mathbb{1}((G, \rho) \in \mathscr{A})]}{\mathbb{E}[\operatorname{deg}(\rho)]}
$$


for every Borel set $\mathscr{A} \subseteq \mathcal{G}_{\bullet}$. Then $\mathbb{P}_{\text {rev }}$ is reversible. Conversely, if $\mathbb{P}$ is a reversible probability measure on $\mathcal{G}_{\bullet}$ with $\mathbb{P}(\operatorname{deg}(\rho)>0)=1$, then biasing $\mathbb{P}$ by $\operatorname{deg}(\rho)^{-1}$ yields a unimodular probability measure on $\mathcal{G}_{\bullet}$.

\subsection{Ergodicity}

A probability measure $\mathbb{P}$ on $\mathcal{G} \bullet$ is said to be ergodic if $\mathbb{P}(\mathscr{A}) \in\{0,1\}$ for every event $\mathscr{A} \subseteq \mathcal{G}$. that is invariant to changing the root in the sense that

$$
(G, \rho) \in \mathscr{A} \Longleftrightarrow(G, v) \in \mathscr{A} \text { for all } v \in V \text {. }
$$

A random rooted graph is said to be ergodic if its law is ergodic. Aldous and Lyons [2, §4] proved that the ergodic unimodular probability measures on $\mathcal{G}$ • are exactly the extreme point of the weakly closed, convex set of unimodular probability measures on. It follows by Choquet theory that every unimodular random rooted graph is a mixture of ergodic unimodular random rooted graphs, meaning that the graph may be sampled by first sampling a random ergodic unimodular probability measure on $\mathcal{G}$. from some distribution, and then sampling from this randomly chosen measure. In particular, to prove almost sure statements about unimodular random rooted graphs, it suffices to consider the ergodic case. Analogous statements hold for unimodular random rooted maps, marked graphs, and marked maps.

\subsection{Duality}

Let $\mathbb{P}$ be a unimodular probability measure on $\mathcal{M}$. such that $M^{\dagger}$ is locally finite $\mathbb{P}$-a.s., and let $(M, \rho)$ be a random rooted map with law $\mathbb{P}_{\text {rev }}$. Conditional on $(M, \rho)$, let $\eta$ be an oriented edge of $M$ sampled uniformly at random from the set $E_{\rho}^{\rightarrow}$, and let $\rho^{\dagger}=\eta^{r}$. We define $\mathbb{P}_{\text {rev }}^{\dagger}$ to the law of the random rooted map $\left(M^{\dagger}, \rho^{\dagger}\right)$.

Proposition 2.4 (Aldous-Lyons [2, Example 9.6]). Let $\mathbb{P}$ be a unimodular probability measure on $\mathcal{M}$. such that $M^{\dagger}$ is locally finite $\mathbb{P}$-a.s. and $\mathbb{P}[\operatorname{deg}(\rho)]<\infty$. Then $\mathbb{P}_{\text {rev }}^{\dagger}$ is a reversible probability measure on $\mathcal{M}$.

Intuitively, under the measure $\mathbb{P}_{\text {rev }}$, the edge $\eta$ is 'uniformly distributed' among the edges of $M$. Since the map sending each edge to its dual is a bijection, it should follow that $\eta^{\dagger}$ is uniformly distributed among the edges of $M^{\dagger}$, making the measure $\mathbb{P}_{\text {rev }}^{\dagger}$ reversible also.

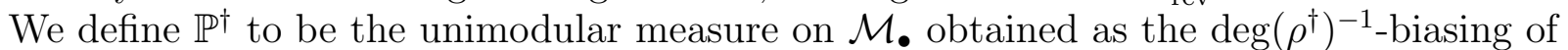
$\mathbb{P}_{\text {rev }}^{\dagger}$. We refer to $\mathbb{P}^{\dagger}$ as the dual of $\mathbb{P}$ and say that $\mathbb{P}$ is self-dual if $\mathbb{P}^{\dagger}=\mathbb{P}$. We write $\mathbb{E}_{\text {rev }}$, $\mathbb{E}_{\mathrm{rev}}^{\dagger}$, and $\mathbb{E}^{\dagger}$ for the associated expectation operators. We may express $\mathbb{P}^{\dagger}$ directly in terms of $\mathbb{P}$ by

$$
\mathbb{P}^{\dagger}\left(\left(M^{\dagger}, \rho^{\dagger}\right) \in \mathscr{A}\right)=\mathbb{E}\left[\sum_{f \perp \rho} \operatorname{deg}(f)^{-1}\right]^{-1} \mathbb{E}\left[\sum_{f \perp \rho} \operatorname{deg}(f)^{-1} \mathbb{1}\left(\left(M^{\dagger}, f\right) \in \mathscr{A}\right)\right]
$$

for every Borel set $\mathscr{A} \subseteq \mathcal{M}$. In particular, we can calculate

$$
\begin{aligned}
\mathbb{E}^{\dagger}\left[\operatorname{deg}\left(\rho^{\dagger}\right)\right] & =\mathbb{E}\left[\sum_{f \perp \rho} \operatorname{deg}(f)^{-1}\right]^{-1} \mathbb{E}\left[\sum_{f \perp \rho} \operatorname{deg}(f)^{-1} \operatorname{deg}(f)\right] \\
& =\mathbb{E}\left[\sum_{f \perp \rho} \operatorname{deg}(f)^{-1}\right]^{-1} \mathbb{E}[\operatorname{deg}(\rho)]<\infty
\end{aligned}
$$


The factor $\mathbb{E}\left[\sum_{f \perp \rho} \operatorname{deg}(f)^{-1}\right]^{-1}$ may be thought of as the ratio of the number of vertices of $M$ to the number of faces of $M$.

Example 2.5. Let $M$ be a finite map and let $\mathbb{P}$ be the law of the unimodular random rooted map $(M, \rho)$ where $\rho$ is a vertex of $M$ chosen uniformly at random. Then $\mathbb{P}^{\dagger}$ is the law of the unimodular random rooted map $\left(M^{\dagger}, \rho^{\dagger}\right)$ obtained by rooting the dual $M^{\dagger}$ of $M$ at a uniformly chosen face $\rho^{\dagger}$ of $M$.

\section{Percolations and Invariant Amenability}

\subsection{Markings and Percolations}

Let $(G, \rho)$ be a unimodular random rooted graph with law $\mathbb{P}$. Given a random rooted graph $(G, \rho)$ and a polish space $\mathbb{X}$, an $\mathbb{X}$-marking of $(G, \rho)$ is a random assignment $m: E \cup V \rightarrow \mathbb{X}$ of marks to the edges and vertices of $(G, \rho)$ (possibly defined on some larger probability space) such that the random rooted marked graph $(G, \rho, m)$ is unimodular. A percolation on $(G, \rho)$ is a $\{0,1\}$-marking of $(G, \rho)$, which we think of as a random subgraph of $G$ consisting of the open edges satisfying $\omega(e)=1$, and open vertices satisfying $\omega(v)=1$. We assume without loss of generality that if an edge is open then so are both of its endpoints. We call $\omega$ a bond percolation if $\omega(v)=1$ for every vertex $v \in V$ almost surely. The cluster $K_{\omega}(v)$ of $\omega$ at the vertex $v$ is the connected component of $v$ in $\omega$. A percolation is said to be finitary if all of its clusters are finite almost surely. A percolation is said to be connected if all open vertices form a single cluster almost surely.

Let us gather here the following simple and useful technical lemmas concerning markings and percolations.

Lemma 3.1. Let $(G, \rho, m)$ be a unimodular random marked graph. Let $\omega$ be a percolation on $(G, \rho, m)$ and let $\left.m\right|_{K_{\omega}(\rho)}$ denote the restriction of $m$ to $K_{\omega}(\rho)$. Then the conditional law of $\left(K_{\omega}(\rho), \rho,\left.m\right|_{\left.\left.K_{\omega}\right) \rho\right)}\right)$ given that $\omega(\rho)=1$ is also unimodular. In particular, if $\omega$ is finitary, then $\rho$ is uniformly distributed on its cluster.

Proof. Let $f$ be a mass transport, and let $g$ be the mass transport

$$
g(G, u, v, m, \omega):=\mathbb{1}\left(v \in K_{\omega}(u)\right) f\left(K_{\omega}(u), u, v,\left.m\right|_{K_{\omega}(u)}\right) .
$$

Applying the mass transport principle for $(G, \rho, \omega)$, we have

$$
\begin{aligned}
\mathbb{E} \sum_{v \in K_{\omega}(\rho)} f\left(K_{\omega}(\rho), \rho, v,\left.m\right|_{K_{\omega}(\rho)}\right) & =\mathbb{E} \sum_{v \in V(G)} g(G, \rho, v, m, \omega)=\mathbb{E} \sum_{v \in V(G)} g(G, v, \rho, m, \omega) \\
& =\mathbb{E} \sum_{v \in K_{\omega}(\rho)} f\left(K_{\omega}(\rho), v, \rho,\left.m\right|_{K_{\omega}(\rho)}\right) .
\end{aligned}
$$

The next two lemmas are proved by abstract probabilistic arguments. They state (roughly) that multiple markings on a graph can be combined, and that a marking on a percolation cluster can be extended to a marking on the entire graph.

Lemma 3.2 (Coupling markings I). Let $(G, \rho)$ be a unimodular random rooted graph and let $\left\{m_{i}: i \in I\right\}$ be a set of markings of $(G, \rho)$ indexed by a countable set I with mark spaces $\left\{\mathbb{X}_{i}: i \in I\right\}$. Then there exists a $\prod_{i \in I} \mathbb{X}_{i}$-marking $m$ of $(G, \rho)$ such that $\left(G, \rho, \pi_{i} \circ m\right)$ and 
$\left(G, \rho, m_{i}\right)$ have the same distribution for all $i \in I$, where $\pi_{i}$ denotes the projection of $\prod_{i \in I} \mathbb{X}_{i}$ onto $\mathbb{X}_{i}$ for each $i \in I$.

Proof. One such marking is given by first sampling $(G, \rho)$ and then sampling the markings $m_{i}$ independently from their conditional distributions given $(G, \rho)$. The details of this construction are are omitted.

Lemma 3.3 (Coupling markings II). Let $(G, \rho)$ be a unimodular random rooted graph, let $\omega$ be a connected percolation on $(G, \rho)$, and let $m$ be an $\mathbb{X}$-marking of the unimodular random rooted graph given by sampling $\left(K_{\omega}(\rho), \rho\right)$ conditional on $\omega(\rho)=1$. Then there exists a $\mathbb{X}$-marking $\hat{m}$ of $(G, \rho)$ such that the laws of $\left(K_{\omega}(\rho), \rho,\left.\hat{m}\right|_{K_{\omega}(\rho)}\right)$ and $\left(K_{\omega}(\rho), \rho, m\right)$ coincide.

Proof. One such marking is given as follows: First sample $\left(K_{\omega}(\rho), \rho\right)$ from its conditional distribution given $\omega(\rho)=1$. Then, independently, sample $(G, \rho)$ and $m$ independently conditional on $\left(K_{\omega}(\rho), \rho\right)$. Extend $m$ to the vertices and edges of $G$ not present in $\omega$ by setting $m$ to be some constant $x_{0} \in \mathbb{X}$ on those vertices. This yields the law of a random rooted marked graph $(G, \rho, \omega, m)$ in which the root always satisfies $\omega(\rho)=1$.

Now, for each vertex $u$ of $G$, let $v(u)$ be chosen uniformly from the set of vertices of $\omega$ closest to $u$, independently from everything else. (In particular, $v(u)=u$ if $\omega(u)=1$.) Note that (by the mass transport principle), the expectation of $|\{u: v(u)=\rho\}|$ is $\mathbb{P}(\rho \in \omega)^{-1}$ and so is finite. Sample the marked graph $(G, \rho, \omega, m)$ biased by $|\{u: v(u)=\rho\}|$, and let $\rho^{\prime}$ be uniform in $\{u: v(u)=\rho\}$. Then $\left(G, \rho^{\prime}, \omega, m\right)$ yields the desired coupling.

\subsection{Amenability}

Recall that the (edge) Cheeger constant of an infinite graph $G=(V, E)$ is defined to be

$$
\mathbf{i}_{E}(G)=\inf \left\{\frac{\left|\partial_{E} W\right|}{|W|}: W \subset V \text { finite }\right\}
$$

where $\partial_{E} W$ denotes the set of edges with exactly one end in $W$. The graph is said to be amenable if its Cheeger constant is zero and nonamenable if it is positive.

Nonamenability is often too strong a condition to hold for random graphs. For example, it is easily seen that every infinite cluster of a Bernoulli percolation on a nonamenable Cayley graph is almost surely amenable, since the cluster will contain atypical 'bad regions' that have small expansion. In light of this, Aldous and Lyons introduced the following weakened notion of nonamenability for unimodular random graphs. (Another way to overcome this issue is to use anchored expansion, which is less relevant in our setting. See [2] for a comparison of the two notions.) The invariant Cheeger constant of an ergodic unimodular random rooted graph $(G, \rho)$ is defined to be

$$
\mathrm{i}^{\text {inv }}(G, \rho)=\inf \left\{\mathbb{E}\left[\frac{\left|\partial_{E} K_{\omega}(\rho)\right|}{\left|K_{\omega}(\rho)\right|}\right]: \omega \text { a finitary percolation on } G\right\} .
$$

(This is a slight abuse of notation: $\mathrm{i}^{\mathrm{inv}}(G, \rho)$ is really a function of the law of $(G, \rho)$.) An ergodic unimodular random rooted graph $(G, \rho)$ is said to be invariantly amenable if $\mathbf{i}^{i n v}(G, \rho)=0$ and invariantly nonamenable otherwise. More generally, we say that a unimodular random rooted graph is invariantly amenable if its ergodic decomposition is supported on invariantly amenable unimodular random rooted graphs, and invariantly nonamenable if its ergodic decomposition is supported on invariantly nonamenable unimodular random rooted graphs. This is a property of the law of $(G, \rho)$ and not of an individual graph. 
Invariant amenability and nonamenability is defined similarly for ergodic unimodular random rooted maps, marked graphs and marked maps.

Let $(G, \rho)$ be a unimodular random rooted graph and let $\omega$ be a finitary percolation on $G$. Let $\operatorname{deg}_{\omega}(\rho)$ denote the degree of $\rho$ in $\omega$ and let

$$
\alpha(G, \rho)=\sup \left\{\mathbb{E}\left[\operatorname{deg}_{\omega}(\rho)\right]: \omega \text { a finitary percolation on } G\right\}
$$

An easy application of the mass transport principle [2, Lemma 8.2] shows that for any finitary percolation

$$
\mathbb{E}\left[\operatorname{deg}_{\omega}(\rho)\right]=\mathbb{E}\left[\frac{\sum_{v \in K_{\omega}(\rho)} \operatorname{deg}_{\omega}(v)}{\left|K_{\omega}(\rho)\right|}\right]=\mathbb{E}\left[\frac{\sum_{v \in K_{\omega}(\rho)} \operatorname{deg}(v)-\left|\partial_{E} K_{\omega}(\rho)\right|}{\left|K_{\omega}(\rho)\right|}\right] .
$$

It follows that, if $\mathbb{E}[\operatorname{deg}(\rho)]<\infty$,

$$
\mathbf{i}^{\text {inv }}(G, \rho)=\mathbb{E}[\operatorname{deg}(\rho)]-\alpha(G, \rho) .
$$

Similarly, if $(G, \rho)$ is an ergodic unimodular random rooted graph with $\mathbb{E}[\operatorname{deg}(\rho)]=\infty$, then $\alpha(G, \rho)<\infty$ is a sufficient condition for $\mathbf{i}^{\text {inv }}(G, \rho)$ to be positive.

\subsection{Hyperfiniteness}

A closely related notion to amenability is hyperfiniteness. A unimodular random rooted graph $(G, \rho)$ is said to be hyperfinite if there exists a sequence of percolations $\left(\omega_{i}\right)_{i \geq 1}$ of $(G, \rho)$ such that each of the percolations $\omega_{i}$ is finitary, $\omega_{i} \subseteq \omega_{i+1}$ almost surely, and $\bigcup_{i \geq 1} \omega_{i}=G$ almost surely. We call such a sequence a finitary exhaustion of $(G, \rho)$. The following is standard in the measured equivalence relations literature and was noted to carry through to the unimodular random rooted graph setting by Aldous and Lyons [2].

Theorem 3.4 ([2, Theorem 8.5]). Let $(G, \rho)$ be a unimodular random rooted graph with $\mathbb{E}[\operatorname{deg}(\rho)]<\infty$. Then $(G, \rho)$ is invariantly amenable if and only if it hyperfinite.

We provide a short proof below for completeness.

Proof. First suppose that $(G, \rho)$ is hyperfinite, and let $\left(\omega_{i}\right)_{i>0}$ be a finitary exhaustion of $(G, \rho)$. By the monotone convergence theorem, $\mathbb{E}\left[\operatorname{deg}_{\omega_{n}}(\rho)\right] \rightarrow \mathbb{E}[\operatorname{deg}(\rho)]$, so that

$$
\alpha(G, \rho) \geq \limsup _{i \rightarrow \infty} \mathbb{E}\left[\operatorname{deg}_{\omega_{i}}(\rho)\right]=\mathbb{E}[\operatorname{deg}(\rho)]
$$

and hence $(G, \rho)$ is invariantly amenable. Suppose conversely that $(G, \rho)$ is invariantly amenable. For each $i \geq 1$, there exists a finitary percolation $\omega_{i}$ on $G$ such that $\mathbb{E}[\operatorname{deg}(\rho)-$ $\left.\operatorname{deg}_{\omega_{i}}(\rho)\right] \leq 2^{-i}$. By Lemma 3.2, these can be coupled. For each $i \geq 1$, let $\hat{\omega}_{i}=\bigcap_{j \geq i} \omega_{j}$. Then $\hat{\omega}_{i}$ is an increasing sequence of finitary percolations. Furthermore, by construction,

$$
\mathbb{E}\left[\operatorname{deg}(\rho)-\operatorname{deg}_{\hat{\omega}_{i}}(\rho)\right] \leq \sum_{j \geq i} \mathbb{E}\left[\operatorname{deg}(\rho)-\operatorname{deg}_{\omega_{i}}(\rho)\right] \leq 2^{-i+1}
$$

By Borel-Cantelli, $\rho$ is in the interior of its $\omega_{i}$-cluster for all sufficiently large $i$ almost surely. It follows by unimodularity that $\bigcup \hat{\omega}_{i}=G$, so that $\left(\hat{\omega}_{i}\right)_{i \geq 0}$ is a finitary exhaustion of $(G, \rho)$.

Remark. Invariantly amenable unimodular random rooted graphs are always hyperfinite whether or not $\mathbb{E}[\operatorname{deg}(\rho)]<\infty$. However, the graph obtained by replacing each edge of the canopy tree (i.e., the Benjamini-Schramm limit of the balls in a 3-regular tree) at height $n$ by $2^{n}$ parallel edges is hyperfinite but nonamenable (since we use edge boundaries). 
Corollary 3.5 (Theorem 1, (2) implies (7)). Let $(M, \rho)$ be a hyperfinite ergodic random rooted map. Then $(M, \rho)$ is a Benjamini-Schramm limit of finite planar maps.

Proof. Let $\left(\omega_{i}\right)_{i \geq 1}$ be a finitary exhaustion of $(M, \rho)$. Let $M_{i}$ be the finite map with underlying graph $K_{\omega_{i}}(\rho)$ and map structure inherited from $M$. Then $\left(M_{i}, \rho_{i}\right)$ is a finite unimodular random rooted map, with the root is uniform in $M_{i}$ (by By Lemma 3.1). Moreover, $\left(M_{i}, \rho\right)$ converges to $(M, \rho)$ almost surely and hence also in distribution.

Theorem 3.6 (Theorem 1: (7) implies (2)). Let $(M, \rho)$ be an ergodic unimodular random planar map that is a Benjamini-Schramm limit of finite planar maps. Then $(M, \rho)$ is hyperfinite.

This is a corollary to the Lipton-Tarjan planar separator theorem [58]; see [4] for details. Similarly, it is possible to deduce that item (8) of Theorem 1 implies item (2) as an application of the low-genus separator theorem of Gilbert, Hutchinson, and Tarjan [36].

\subsection{Ends}

Recall that an infinite connected graph $G=(V, E)$ is said to be $k$-ended if $k$ is minimal such that for every finite set $W$ of vertices in $G$, the graph induced by the complement $V \backslash W$ has at most $k$ distinct infinite connected components. In particular, an infinite tree is one-ended if it does not contain a simple bi-infinite path, and is two-ended if it contains a unique bi-infinite path.

The following proposition, due primarily to Aldous and Lyons [2], connects the number of ends of a unimodular random rooted graph to invariant amenability.

Proposition 3.7 (Ends and Amenability $[2,4])$. Let $(G, \rho)$ be an infinite ergodic unimodular random rooted graph with $\mathbb{E}[\operatorname{deg}(\rho)]<\infty$. Then $G$ has either one, two, or infinitely many ends almost surely. If $G$ has infinitely many ends, then $(G, \rho)$ is invariantly nonamenable. If $G$ has two ends almost surely, then it is almost surely recurrent and $(G, \rho)$ is invariantly amenable.

Similarly, a connected topological space $X$ is said to be $k$-ended if over all compact subsets $K$ of $X$, the complement $X \backslash K$ has a maximum of $k$ connected components that are not precompact.

Lemma 3.8. Let $M$ be a map. Then the underlying graph of $M$ has at least as many ends as the associated surface $S=S(M)$.

Note that $M$ can have more ends than $S(M)$. For example the doubly infinite path has 2 ends, and the 3-regular tree is infinitely-ended, but both associated surfaces are homeomorphic to the plane and hence one-ended.

Proof. For each compact subset $K$ of $S$, let $W_{K}$ be the set of vertices of $M$ that are adjacent to an edge intersecting $K$. Since every face of $M$ in $S$ is a topological disc, each nonprecompact connected component of $S \backslash K$ contains infinitely many edges of $M$, and there are no connections in $M \backslash W_{K}$ between these components. Thus $M \backslash W_{K}$ has at least as many infinite connected components as $S \backslash K$ has non-precompact connected components.

Finally, let us note the following simple topological fact.

Lemma 3.9. Let $M$ be a map. Then the underlying graph of $M$ has at least as many ends as $M$ has faces of infinite degree. 


\subsection{Unimodular Couplings}

Let $\left(G_{1}, \rho_{1}\right)$ and $\left(G_{2}, \rho_{2}\right)$ be unimodular random rooted graphs. A unimodular coupling of $\left(G_{1}, \rho_{1}\right)$ and $\left(G_{2}, \rho_{2}\right)$ is a unimodular random rooted $\{0,1\}^{2}$-marked graph $\left(G, \rho, \omega_{1}, \omega_{2}\right)$ such that $\omega_{1}$ and $\omega_{2}$ are both connected and the law of the subgraph $\left(\omega_{i}, \rho\right)$ conditioned on the event that $\omega_{i}(\rho)=1$ is equal to the law of $\left(G_{i}, \rho_{i}\right)$ for each $i$. We say that two unimodular random rooted graphs $\left(G_{1}, \rho_{1}\right)$ and $\left(G_{2}, \rho_{2}\right)$ are coupling equivalent if they admit a unimodular coupling. (It is not difficult to show that this is an equivalence relation, arguing along similar lines to Lemmas 3.2 and 3.3.) Coupling equivalence is closely related to the notion of two graphings generating the same equivalence relation in the theory of measured equivalence relations (see [2, Example 9.9]). Unimodular maps are defined to be coupling equivalent if the underlying graphs are. Equivalence of marked graphs and maps is defined similarly.

Example 3.10. Let $(G, \rho)$ be a unimodular random rooted graph and let $\omega$ be a connected bond percolation on $G$. Then $(G, \rho)$ and $(\omega, \rho)$ are coupling equivalent.

Example 3.11. Let $(M, \rho)$ be a unimodular random rooted map with locally finite dual. Then $(M, \rho)$ is coupling equivalent to its unimodular dual: both $(M, \rho)$ and its dual can be represented as percolations of the graph formed by combining $M$ and its dual by adding a vertex wherever a primal edge crosses a dual edge, as well as keeping the original primal and dual edges. (see [2, Example 9.6] for a similar construction). Note that the graph containing $M$ and $M^{\dagger}$ is not a map itself.

The main use of unimodular coupling will be that hyperfiniteness and strong soficity are both preserved under unimodular coupling. The corresponding statements for measured equivalence relations are due to Elek and Lippner [33].

Proposition 3.12. Let $\left(G_{1}, \rho_{1}\right)$ and $\left(G_{2}, \rho_{2}\right)$ be coupling equivalent unimodular random rooted graphs. Then $\left(G_{2}, \rho_{2}\right)$ is hyperfinite if and only if $\left(G_{1}, \rho_{1}\right)$ is.

Proof. It suffices to prove that if $(G, \rho)$ is a unimodular random rooted graph and $\omega$ is a connected percolation on $(G, \rho)$, then $(G, \rho)$ is hyperfinite if and only if $(\omega, \rho)$ is hyperfinite.

Suppose first that $(G, \rho)$ is hyperfinite and let $\left(\tilde{\omega}_{i}\right)_{i \geq 1}$ be a finitary exhaustion of $G$. By Lemma 3.2, we may couple $\omega$ and $\left(\omega_{i}\right)_{i \geq 1}$ so that $\left(G, \rho,\left(\omega,\left(\tilde{\omega}_{i}\right)_{i \geq 1}\right)\right)$ is unimodular. Under such a coupling, $\left(\omega \cap \tilde{\omega}_{i}\right)_{i \geq 1}$ is a finitary exhaustion of $(\omega, \rho)$, and consequently $(\omega, \rho)$ is hyperfinite.

Suppose conversely that $(\omega, \rho)$ is hyperfinite, and let $\left(\tilde{\omega}_{i}\right)_{i \geq 1}$ be a finitary exhaustion of $(\omega, \rho)$. By Lemma 3.3, we may assume that $\left(G, \rho, \omega,\left(\tilde{\omega}_{i}\right)_{i \geq 1}\right)$ is unimodular. For each vertex $v$ of $G$, let $w(v)$ be a chosen uniformly from the set of vertices of $\omega$ minimising the graph distance to $v$ in $G$. For each $i$, define a subgraph $\hat{\omega}_{i}$ of $G$ by

$$
\hat{\omega}_{i}(u, v)=1 \Longleftrightarrow w(u) \text { and } w(v) \text { are connected in } \tilde{\omega}_{i} .
$$

Then $\left(\hat{\omega}_{i}\right)_{i \geq 1}$ is a finitary exhaustion of $(G, \rho)$.

Remark. It can be deduced from Proposition 3.12 and [2, Theorem 8.9] that a unimodular random rooted graph with finite expected degree is hyperfinite if and only if it is coupling equivalent to $\mathbb{Z}$.

One useful application of Proposition 3.12 is the following. 
Lemma 3.13. Let $(M, \rho)$ be an ergodic unimodular random rooted map. The number of infinite degree faces is either $0,1,2$, or $\infty$, and if it is 1 or 2 then $(M, \rho)$ is hyperfinite.

Proof. By applying Proposition 3.7 and Lemma 3.9, it suffices to prove that if $M$ has a non-zero but finite number of infinite degree faces almost surely, then $(M, \rho)$ is hyperfinite. Suppose that $M$ has finitely many infinite degree faces almost surely. Conditional on $(M, \rho)$, choose one infinite face, $f$, uniformly at random. The boundary of this face gives a coupling of $M$ and $\mathbb{Z}$, except for the fact that this boundary need not be simple.

If for every vertex $v$ of $f$ there exists a vertex $v^{\prime}$ of $f$ such that $v$ is contained in a finite component of $M \backslash\left\{v^{\prime}\right\}$, then inductively there exists a sequence of vertices $\left(v_{n}\right)_{n \geq 1}$ of $M$ such that $v_{n}$ is contained in a finite connected component of $M \backslash\left\{v_{n+1}\right\}$ for every $n \geq 1$. We deduce in this case that $M$ is hyperfinite by taking as a finitary exhaustion the sequence of random subgraphs $\left(\tilde{\omega}_{m}\right)_{m \geq 1}$ induced by the standard monotone coupling of Bernoulli $1-1 / m$ site percolations on $M$. All clusters of $\omega_{m}$ are almost surely finite due to the existence of the cutpoints $\left(v_{n}\right)_{n \geq 1}$.

Otherwise, define a percolation $\omega$ on $(M, \rho)$ as follows. Vertices of $f$ that are not separated from infinity by any other vertex of $f$ are open, as are the edges of $f$ between such vertices, and no other vertices or edges. Then $\omega$ is connected and is isomorphic to the bi-infinite line graph $\mathbb{Z}$. It follows that $(G, \rho)$ is coupling equivalent to $(\mathbb{Z}, 0)$ and hence hyperfinite by Proposition 3.12

\subsection{Vertex Extremal Length and Recurrence of Subgraphs}

Vertex extremal length was introduced by He and Schramm [44] and is closely connected to circle packing. Let $G$ be an infinite graph. For a vertex $v$ of $G$, the vertex extremal length from $v$ to infinity is defined to be

$$
\operatorname{VEL}_{G}(v, \infty)=\sup _{m} \frac{\inf _{\gamma: v \rightarrow \infty} m(\gamma)^{2}}{\|m\|^{2}}
$$

where the supremum is over measures $m$ on the vertex set of $G$ such that $\|m\|^{2}=\sum m(u)^{2}<$ $\infty$, and the infimum is over paths $\gamma$ from $v$ to $\infty$ in $G$. A connected graph is said to be VEL parabolic if $\operatorname{VEL}(v \rightarrow \infty)=\infty$ for some vertex $v$ of $G$ (and hence for every vertex), and VEL hyperbolic otherwise. As noted in [44], if $G$ is VEL parabolic then there exists a vertex measure $m$ where $\|m\|<\infty$ and $m(\gamma)=\infty$ for every path $\gamma: v \rightarrow \infty$.

The edge extremal length from $v$ to infinity is defined in the same way except that the measure $m$ is on the edges of $G$, and is equal to the effective resistance from $v$ to infinity. In particular, $G$ is recurrent if and only if $\mathcal{R}_{\text {eff }}(v, \infty)=\operatorname{EEL}(v, \infty)=\infty$ [31]. See [60] for further background.

The VEL type is monotone in the sense that subgraphs of VEL parabolic graphs are also VEL parabolic. Consequently, the following two lemmas give the corresponding implications in Theorem 1.

Lemma 3.14 ([44], Theorem 1, (17) implies (5)). Let $G$ be a locally finite, connected graph. If $G$ is VEL hyperbolic, then it is transient. If $G$ is transient and has bouneded degrees then it is VEL hyperbolic.

For trees, as for graphs with bounded degrees, VEL parabolicity is equivalent to recurrence:

Lemma 3.15 (Theorem 1, (17) implies (6)). Let $T$ be a tree. Then $T$ is transient if and only if it is VEL hyperbolic. 
Proof. If $T$ is VEL hyperbolic then it is transient by [44]. Suppose conversely that $T$ is VEL parabolic. Then there is a vertex measure $m$ on $T$ with $\|m\|<\infty$ and $m(\gamma)=\infty$ for every infinite path from $v$. For each edge $e$ of $T$, let $u(e)$ be the endpoint of $e$ farther from $v$, and define an edge measure $\hat{m}$ on $T$ by setting $\hat{m}(e)=m(u(e))$ for every edge $e$ of $T$. Then $\|\hat{m}\|^{2}=\|m\|^{2}-m(v)^{2}<\infty$, and every simple path $\gamma: v \rightarrow \infty$ has $\hat{m}(\gamma)=m(\gamma)-m(v)=\infty$. Thus $\mathcal{R}_{\text {eff }}(v, \infty)=\infty$ and $G$ is recurrent.

Benjamini and Schramm [20] used circle packing to prove the following remarkable theorem. See [4] for an alternative proof, and $[39,6]$ for related results establishing recurrence in certain unbounded degree cases.

Theorem 3.16 ([20]; Theorem 1, (7) implies (17)). Let $(M, \rho)$ be a Benjamini-Schramm limit of finite planar maps. Then $M$ is VEL parabolic almost surely. In particular, if $M$ has bounded degrees, then it is almost surely recurrent for simple random walk.

We finish this section with the following theorem.

Theorem 3.17 (Benjamini, Lyons and Schramm [14]; Aldous and Lyons [2]: Theorem 1, (3) and (4) each imply $(2))$. Let $(G, \rho)$ be an invariantly nonamenable unimodular random rooted graph. Then there exists a percolation $\omega$ on $G$ such that a.s. every connected component of $\omega$ is nonamenable, and there exists a constant $M$ such that $\operatorname{deg}(v) \leq M$ for every vertex $v$ of $G$ such that $\omega(v)=1$. Furthermore, the percolation $\omega$ can be taken to be a forest.

In particular, if a unimodular random map is nonamenable then it contains nonamenable sub-trees with bounded degrees, which in turn are VEL hyperbolic. This theorem has been very useful for studying random walks on invariantly nonamenable unimodular random rooted graphs; see [4, Section 5.1]. See [4, Section 3.3.1] for a complete proof of the first part of the theorem (in which $\omega$ is not taken to be a forest).

\section{Curvature}

In this section, we study the average curvature of a unimodular random rooted map, and its basic properties. Essentially, the average curvature is the average Gaussian curvature per vertex when the map is embedded on a Riemannian manifold. We begin by giving a combinatorial definition of the average curvature. We then show that the average curvature is a canonical quantity associated to the random map, in the sense that any unimodular embedding the map in a Riemannian manifold (satisfying certain integrability conditions) will result in the same average curvature.

Recall that the internal angles of a regular $k$-gon are given by $(k-2) \pi / k$. We define the angle sum at a vertex $v$ of a map $M$ to be

$$
\theta(v)=\theta_{M}(v)=\sum_{f \perp v} \frac{\operatorname{deg}(f)-2}{\operatorname{deg}(f)} \pi
$$

(Recall that this sum counts each face with the appropriate multiplicity.) This definition extends to maps with infinite faces, with the convention that $(\infty-2) / \infty=1$. In the case that every face of $M$ has degree at least 3, embed $M$ in a manifold by gluing together regular polygons (with the $\infty$-gon being the upper half-space $\{x+i y \in \mathbb{C}: y>0\}$ with edges $\{[n, n+1]: n \in \mathbb{Z}\})$. We then have that $\theta(v)$ is the total angle of the corners at $v$. 

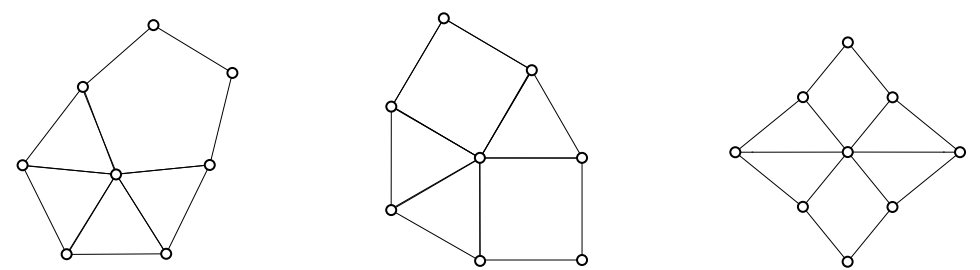

Figure 4. The centre vertices have curvature $\pi / 15,0$ and $-\pi / 3$ (left to right).

Of course, in general $M$ cannot necessarily be drawn in the Euclidean plane so that all faces are regular polygons, and the angle sum at a vertex of $M$ need not be $2 \pi$. We define the curvature of $M$ at the vertex $v$ to be the angle sum deficit

$$
\kappa(v)=\kappa_{M}(v)=2 \pi-\theta(v) .
$$

See Figure 4 for examples. This combinatorial definition of curvature is well-known and (in the deterministic setting) has been extensively studied in the literature, see e.g. [26, 3, 45] and references therein. We define the average curvature of a unimodular random rooted map $(M, \rho)$, denoted $\mathbb{K}(M, \rho)$, to be the expected curvature at the root:

$$
\mathbb{K}(M, \rho)=\mathbb{E}[\kappa(\rho)]=2 \pi-\mathbb{E}\left[\sum_{f \perp \rho} \frac{\operatorname{deg}(f)-2}{\operatorname{deg}(f)} \pi\right] .
$$

Note that if $\mathbb{E}[\operatorname{deg}(\rho)]$ is finite then $\mathbb{K}(M, \rho)$ is also finite.

Example 4.1 (Finite Maps). Let $M$ be a finite map and let $\rho$ be a vertex of $M$ chosen uniformly at random. Then a simple calculation using Euler's formula gives that

$$
\mathbb{K}(M, \rho)=\frac{1}{|V|} \sum_{v \in V} \kappa(v)=\frac{2 \pi}{|V|}(2-2 \operatorname{genus}(M)) .
$$

If $S$ is a Riemannian surface of genus $g$, and $\kappa(\cdot)$ is the associated Gaussian curvature, then the Gauss-Bonnet theorem implies that $\int_{S} \kappa(x) d x=2 \pi(2-2 g)$. Consequently, for an arbitrary Riemannian metric on $S(M)$ we have

$$
\mathbb{K}(M, \rho)=\frac{1}{|V|} \int_{S} \kappa(x) d x .
$$

Thus, our definition of curvature agrees with the average Gaussian curvature per vertex, and is independent of the choice of metric. This is a special case of Theorem 4.10 below.

Example 4.2 (k-angulations). If $M$ is a $k$-angulation (i.e., every face of $M$ has degree $k$ ), then

$$
\theta(v)=\frac{k-2}{k} \operatorname{deg}(v) \pi
$$

for every vertex $v$ of $M$. Consequently, if $(M, \rho)$ is a unimodular random $k$-angulation, then

$$
\mathbb{K}(M, \rho)=\left(2-\frac{k-2}{k} \mathbb{E}[\operatorname{deg}(\rho)]\right) \pi .
$$

A special case corresponding to $k=\infty$, is when $M$ is an infinite plane tree, the angle sum at a vertex $v$ of $M$ is simply $\theta(v)=\pi \operatorname{deg}(v)$, and the average curvature is $\pi(2-\mathbb{E} \operatorname{deg}(\rho))$. 
Example 4.3 (Curvature of the dual measure). Let $\mathbb{P}$ be a unimodular probability measure on $\mathcal{M}$. such that $\mathbb{P}[\operatorname{deg}(\rho)]<\infty$ and $M$ has locally finite dual $\mathbb{P}$-a.s., and let $\mathbb{P}^{\dagger}$ be the dual measure as defined above. By (2.2),

$$
\begin{aligned}
\mathbb{K}\left(M^{\dagger}, \rho^{\dagger}\right) & =2 \pi-\mathbb{E}^{\dagger}\left[\sum_{v \perp \rho^{\dagger}} \frac{\operatorname{deg}(v)-2}{\operatorname{deg}(v)}\right] \pi \\
& =2 \pi-\mathbb{E}\left[\sum_{f \perp \rho} \frac{1}{\operatorname{deg}(f)}\right]^{-1} \mathbb{E}\left[\sum_{f \perp \rho} \frac{1}{\operatorname{deg}(f)} \sum_{v \perp f} \frac{\operatorname{deg}(v)-2}{\operatorname{deg}(v)}\right] \pi .
\end{aligned}
$$

Applying the mass transport principle yields that

$$
\mathbb{K}\left(M^{\dagger}, \rho^{\dagger}\right)=2 \pi-\pi \mathbb{E}\left[\sum_{f \perp \rho} \frac{1}{\operatorname{deg}(f)}\right]^{-1} \mathbb{E}\left[\sum_{f \perp \rho} \frac{1}{\operatorname{deg}(f)} \sum_{v \perp f} \frac{\operatorname{deg}(\rho)-2}{\operatorname{deg}(\rho)}\right] .
$$

This can be rearranged to give

$$
\mathbb{K}\left(M^{\dagger}, \rho^{\dagger}\right)=\mathbb{E}\left[\sum_{f \perp \rho} \frac{1}{\operatorname{deg}(f)}\right]^{-1} \mathbb{K}(M, \rho)
$$

Recall that $\mathbb{E}\left[\sum_{f \perp \rho} \frac{1}{\operatorname{deg}(f)}\right]^{-1}$ is interpreted as the ratio between vertices of $M$ to vertices of $M^{\dagger}$. While the average curvature per vertex is changed, this is only since the density of vertices is changed. In particular, the average curvature of $(M, \rho)$ under $\mathbb{P}$ has the same sign as that of $\left(M^{\dagger}, \rho^{\dagger}\right)$ under $\mathbb{P}^{\dagger}$.

Example 4.4 (Self-dual maps). Let $(M, \rho)$ be a self-dual unimodular random rooted map. Then (2.3) implies that $\mathbb{E}\left[\sum_{f \perp \rho} \operatorname{deg}(f)^{-1}\right]=1$ and so

$$
\begin{aligned}
\mathbb{K}(M, \rho) & =2 \pi-\mathbb{E}\left[\sum_{f \perp \rho} \frac{\operatorname{deg}(f)-2}{\operatorname{deg}(f)}\right] \pi \\
& =2 \pi-\mathbb{E}[\operatorname{deg}(\rho)] \pi+2 \mathbb{E}\left[\sum_{f \perp \rho} \operatorname{deg}(f)^{-1}\right] \pi=4 \pi-\mathbb{E}[\operatorname{deg}(\rho)] \pi .
\end{aligned}
$$

\subsection{Curvature of submaps}

Let $M$ be a map with underlying graph $G$, and let $z$ be a proper embedding of $M$ into an orientable surface $S$. A submap of $M$ is a map represented by a triple $(H, S, z)$, where $H$ is a connected subgraph of $G$ such that the restriction of $z$ to $H$ is a proper embedding of $H$ into $S$ (in particular, the faces are topological discs). If $M$ is simply connected, then every connected subgraph of $G$ is also a submap of $M$. (This may fail if $M$ is not simply connected.)

Since the average curvature is curvature per vertex, it is not surprising that changing the map without adding or removing vertices does not change $\mathbb{K}$. Indeed, in the case of a finite map, deleting edges gives an embedding of the submap on the same surface, with the same total and hence same average curvature. Recall that we call a percolation $\omega$ on $(M, \rho)$ a bond percolation if $\omega(v)=1$ for every vertex $v$ of $M$ almost surely. 
Proposition 4.5 (Curvature of random submaps). Let $(M, \rho)$ be a unimodular random rooted map with $\mathbb{E}[\operatorname{deg}(\rho)]<\infty$ and let $\omega$ be a connected bond percolation on $(M, \rho)$ that is almost surely a submap of $M$. Then

$$
\mathbb{K}(\omega, \rho)=\mathbb{K}(M, \rho) .
$$

Remark. It is straightforward to extend of Proposition 4.5 to submaps $\omega$ of $(M, \rho)$, that do not include every vertex. Let $(N, \rho)$ be the unimodular random map obtained from $(\omega, \rho)$ by conditioning on the event $\rho \in \omega$, we have that

$$
\mathbb{K}(N, \rho)=\mathbb{P}(\rho \in \omega)^{-1} \mathbb{K}(M, \rho) .
$$

Proof. First suppose that $M$ and $\omega$ both have a locally finite duals a.s. In this case, every face of $\omega$ is a union of finitely many faces of $M$. For a face $f$ of $M$, denote by $\hat{f}$ the face of $\omega$ containing $f$.

We define a mass transport in three stages as follows. Each vertex $v$ sends a mass of $(\operatorname{deg}(f)-2) / \operatorname{deg}(f)$ to each face $f$ incident to it, counting with multiplicity as usual. Each face $f$ then sends all the mass it receives to $\hat{f}$. Finally, each face $\hat{f}$ of $\omega$ redistributes the total mass it receives uniformly to its vertices (again, counted with multiplicity). This procedure yields a mass transport in which the mass sent from $u$ to $v$ is

$$
\sum_{u \perp f \subset \hat{f} \perp v} \frac{\operatorname{deg}(f)-2}{\operatorname{deg}(f) \operatorname{deg}(\hat{f})}
$$

where the sum is over faces $f \subset \hat{f}$ containing $u$ and $v$ respectively. In particular, we have

$$
\sum_{v \in V}\left|\sum_{u \perp f \subset \hat{f} \perp v} \frac{\operatorname{deg}(f)-2}{\operatorname{deg}(f) \operatorname{deg}(\hat{f})}\right| \leq \sum_{u \perp f}\left|\frac{\operatorname{deg}(f)-2}{\operatorname{deg}(f)}\right| \leq \operatorname{deg}(u),
$$

so that this mass-transport (which can be negative in the presence of degree 1 faces) meets the integrability requirements needed to apply the signed Mass-Transport Principle.

The total mass sent from a vertex $v$ is $\theta_{M}(v) / 2 \pi$. The total mass passing through a face $f$ of $M$ is $\operatorname{deg}(f)-2$, while the total mass passing through a face $\hat{f}$ of $\omega$ is $\sum_{f \subset \hat{f}} \operatorname{deg}(f)-2$. It is easy to see (by induction or using Euler's formula) that the latter quantity equals $\operatorname{deg}(\hat{f})-2$, and it follows that the total mass received by a vertex $v$ is

$$
\sum_{\hat{f} \perp v} \frac{\operatorname{deg}(\hat{f})-2}{\operatorname{deg}(\hat{f})}=\frac{1}{2 \pi} \theta_{\omega}(v) .
$$

The mass transport principle gives that $\mathbb{E} \theta_{M}(\rho)=\mathbb{E} \theta_{\omega}(\rho)$, establishing the claim in this case.

Next, suppose that $M$ has locally finite dual but that $\omega$ does not. Every edge of $M$ not in $\omega$ separates some face of $\omega$ in two. For each infinite face $\hat{f}$ of $\omega$, let $\left(v_{i}\right)_{i \in \mathbb{Z}}$ be the vertices at the corners of $\hat{f}$ in counterclockwise order. These labels are well defined up to an additive constant. For each $n \geq 1$, define a percolation $\omega_{n}$ by letting $e \in \omega_{n}$ if and only if either $e \in \omega$ or else if $e$ connects vertices $v_{i}$ and $v_{j}$ of some infinite face $\hat{f}$ with $|i-j| \geq n$. Observe that, since $M$ has a locally finite dual, $\omega_{n}$ has locally finite dual for every $n$. It follows from the first case above that $\mathbb{K}\left(\omega_{n}, \rho\right)=\mathbb{K}(M, \rho)$. The sequence of random variables $\left|\theta_{\omega_{n}}(\rho)\right|$ are bounded by $2 \pi \operatorname{deg}(\rho)$, so that $\mathbb{K}\left(\omega_{n}, \rho\right) \rightarrow \mathbb{K}(\omega, \rho)$ as $n \rightarrow \infty$ by the dominated convergence theorem, completing the proof in this case. 


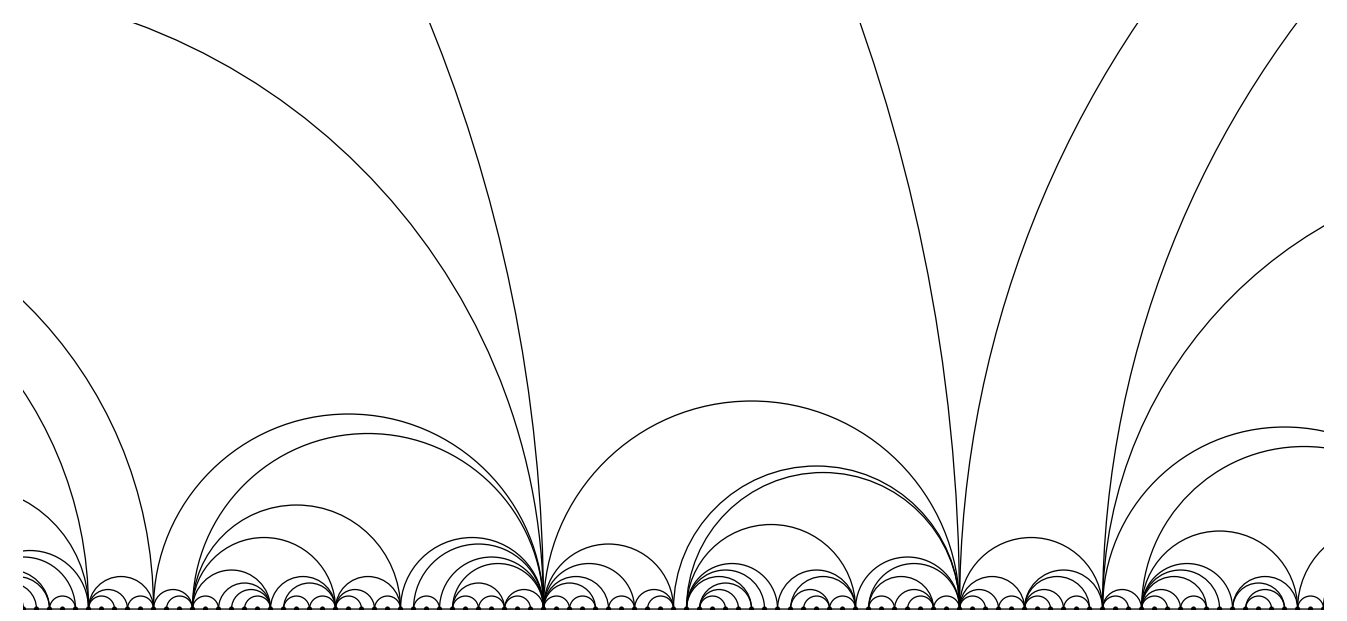

Figure 5. The map $M_{1}$ is defined by filling in each infinite face of $M$ with a system of arcs. This figure demonstrates this procedure applied to one of the infinite faces of $\mathbb{Z}$.

Finally, suppose that $M^{\dagger}$ is not locally finite. We construct a unimodular map $M^{\prime}$ with locally finite dual such that $M$ (and hence also $\omega$ ) is a submap of $M^{\prime}$ with the same vertex set. By the previous case, $\mathbb{K}(M, \rho)=\mathbb{K}\left(M^{\prime}, \rho\right)=\mathbb{K}(\omega, \rho)$. To this end, assign to each corner $a=\left(e, e^{\prime}\right)$ of every infinite face of $M$ an i.i.d. random variable $U_{a}$, uniform in $[0,1]$. For each face $f$ of $M$, add an edge between non-adjacent corners $a$ and $b$, if there is some $t$ so that $U_{a}, U_{b} \leq t$ and every other corner $c$ of $f$ between $a$ and $b$ has $U_{c}>t$. Here, adding an edge between corners means the edge connects the vertices of the corners, and is inserted between the two edges of the corners in the cyclic edge order at each endpoint. It is easy to see that this gives a triangulation of the infinite face of $M$, so that $\left(M^{\prime}\right)^{\dagger}$ is locally finite.

It is easy to see that these edges can be drawn in the face $f$ with no crossings: If $a, b, c, d$ are corners appearing in clockwise order in $f$ and the edge $(a, c)$ is added, then $U_{b}>U_{c}$ and so the edge $(b, c)$ cannot be added. The probability that a corner is connected to another corner at distance $k$ away along the face is $\left(\begin{array}{c}k+1 \\ 2\end{array}\right)^{-1}$, since their labels must be the two smallest of $k+1$ exchangeable labels. Thus, the expected number of edges added at a corner of an infinite face is $\sum 2\left(\begin{array}{c}k+1 \\ 2\end{array}\right)^{-1}=2$. It follows that $\mathbb{E} \operatorname{deg}_{M^{\prime}}(\rho) \leq \mathbb{E} 3 \operatorname{deg}_{M}(\rho)<\infty$, and we conclude by applying the previous case.

Proposition 4.6. $\mathbb{K}$ is upper-semicontinuous on the set of unimodular maps with finite expected degree with respect to the local topology. Explicitly, Let $\left(M_{n}, \rho_{n}\right)$ be a sequence of unimodular random rooted maps with $\mathbb{E}\left[\operatorname{deg}\left(\rho_{n}\right)\right]<\infty$ that converge weakly to a unimodular random rooted map $(M, \rho)$ with $\mathbb{E}[\operatorname{deg}(\rho)]<\infty$. Then

$$
\mathbb{K}(M, \rho) \geq \lim _{n \rightarrow \infty} \mathbb{K}\left(M_{n}, \rho_{n}\right) .
$$

Proof. First suppose that none of the maps $M_{n}$ have any faces of degree 1 . In this case, $\theta_{M_{n}}(v) \geq 0$ for every $v$ and $n \geq 1$, and the claim follows from Fatou's lemma.

Otherwise, we say that a self-loop is redundant if it is contractible in $S(M)$, and all the vertices of $M$ are in the same component of the complement of the loop. Note that if $M$ is simply connected then every self-loop is contractible, and a redundant self-loop may surround other redundant self-loops but no other edge. Let $\hat{M}$ and $\hat{M}_{n}$ be obtained from 
$M$ and $M_{n}$ respectively by deleting all redundant self-loops. Proposition 4.5 implies that $\mathbb{K}(\hat{M}, \rho)=\mathbb{K}(M, \rho)$ and $\mathbb{K}(\hat{M}, \rho)=\mathbb{K}\left(\hat{M}_{n}, \rho_{n}\right)=\mathbb{K}\left(M_{n}, \rho_{n}\right)$ for all $n \geq 1$. Since the operation of removing redundant self-loops is continuous in the weak toplogy, $\left(\hat{M}_{n}, \rho_{n}\right)$ converges weakly to $(M, \rho)$, and the claim follows from Fatou's Lemma as above.

Combining Proposition 4.6 with the equation (4.1) has the following immediate corollary.

Corollary 4.7 (Theorem $1,(8)$ implies $(1))$. Let $(M, \rho)$ be a unimodular random rooted map with $\mathbb{E}[\operatorname{deg}(\rho)]<\infty$ that is a Benjamini-Schramm limit of a sequence of finite maps $\left(M_{n}\right)_{n \geq 1}$ such that

$$
\frac{\operatorname{genus}\left(M_{n}\right)}{\left|V\left(M_{n}\right)\right|} \underset{n \rightarrow \infty}{\longrightarrow} 0 .
$$

Then $\mathbb{K}(M, \rho) \geq 0$.

We will prove in Section 5 that $\mathbb{K}(M, \rho) \leq 0$ for any infinite, simply connected, unimodular random rooted planar map with finite expected degree, so that Benjamini-Schramm limits of low genus finite maps have 0 average curvature.

\subsection{Invariance of the curvature}

In this section, we consider unimodular embeddings of unimodular random rooted maps. We define extend the notion of average curvature to such an embedding and show that, under certain integrability conditions, the average curvature associated to the embedding agrees with the average curvature that we defined combinatorially. This shows that the average curvature is a canonical quantity.

We define a metric surface embedded map (MSEM) to be a locally finite map $M$ together with a proper embedding $z$ of $M$ into an oriented metric surface $S$. A rooted MSEM is a MSEM together with a distinguished root vertex. Two rooted MSEMs are isomorphic if they are isomorphic as rooted maps, and there is an orientation preserving isometry between the two surfaces sending one embedding to the other.

We define the local topology on the set of isomorphism classes of rooted MSEMs by a variation on the local Gromov-Haussdorf topology: Namely, we set the distance between two rooted MSEMs $\left(M_{1}, \rho_{1}, S_{1}, z_{1}\right)$ and $\left(M_{2}, \rho_{2}, S_{2}, z_{2}\right)$ to be $e^{-r}$, where $r$ is maximal such that there is a map isomorphism $\phi$ from the (graph distance) ball of radius $r$ around $\rho_{1}$ in $M_{1}$ to the ball of radius $r$ about $\rho_{2}$ in $M_{2}$ and a correspondence $R$ between the balls of (metric) radius $r$ around $z_{1}\left(\rho_{1}\right)$ and $z_{2}\left(\rho_{2}\right)$ in $S_{1}$ and $S_{2}$ respectively, denoted $B_{1}(r)$ and $B_{2}(r)$, such that if $\left(x, x^{\prime}\right)$ and $\left(y, y^{\prime}\right) \in R$ then $\left|d_{1}(x, y)-d_{2}\left(x^{\prime}, y^{\prime}\right)\right| \leq 1 / r$, and such that if $\phi(e)=e^{\prime}$ then $R$ restricts to a correspondence between the points of $e$ and of $e^{\prime}$ that are contained in the metric balls of radius $r$ around $z_{1}\left(\rho_{1}\right)$ and $z_{2}\left(\rho_{2}\right)$ respectively.

We define doubly rooted MSEMs, the local topology on the set of isomorphism classes of doubly rooted MSEMs, and unimodular random rooted MSEMs similarly to the graph case. It is straightforward (but rather tedious) to encode the structure of a MSEM as a marking of the underlying map of the MSEM, so that all of the usual machinery of unimodularity transports to this setting. If $(M, \rho)$ is a unimodular random rooted map and $(M, S, z, \rho)$ is a unimodular random rooted MSEM with underlying map $(M, \rho)$, we call $(S, z)$ a unimodular embedding of $M$.

In practice, unimodular embeddings often arise as measurable, automorphism equivariant functions of the map, such as the embeddings given by circle packing and the conformal 
embedding. These are easily seen to be unimodular since every mass transport on the associated MSEM is induced by a mass transport on the map.

Example 4.8. Given a map $M$ such that every face of $M$ has degree at least three, a natural way to embed it on a surface is as follows. Associate to each face $f$ of degree $d$ a regular $d$-gon with sides of length 1 . If two faces share an edge, we identify the corresponding edges of the polygons. For infinite faces, the corresponding polygon is a half-plane $\{z \in \mathbb{C}: \operatorname{Im}(z) \geq 0\}$ with edges $\{[n, n+1]: n \in \mathbb{Z}\}$ along the boundary, which we think of as a regular $\infty$-gon. In this surface, there is no curvature on any of the faces, as they are all flat pieces of $\mathbb{R}^{2}$. The surface is also smooth along the edges, as two faces are glued along straight segments of unit length. Thus, all of the curvature of the surface is concentrated on the vertices, and in fact the atom of curvature $\kappa_{s}(v)$ at $v$ (see below) is exactly the value $\kappa(v)$ that we defined combinatorially at the beginning of this section.

Example 4.9 (glued discs). Similarly to the above, we can associate to each face of $M$ of degree $d$ a disc of circumference $d$ (a half-plane if $d=\infty$ ). Split the boundary of each disc to unit length segments, and glue discs along these segments. This is well defined also for maps with faces of degree 1 and 2. In this case, the singular curvature at a vertex is $\kappa_{s}(v)=\pi(2-\operatorname{deg}(v)$, but there is also positive Gaussian curvature supported on the edges.

The two examples above are jointly generalized by constructions based on gluing (possibly random) shapes assigned to faces. In what follows, we restrict ourselves to surfaces that have a smooth Riemannian metric, except possibly for conical singularities at vertices of the map, and assume that the edges of the map are embedded as smooth curves in the surface. We call a unimodular embedding of a map satisfying these conditions a smooth embedding. For example, any construction based on gluing polygons yields a smooth embedding.

Recall a corner is a pair $\left(e, e^{\prime}\right)$ of oriented edges such that $e^{\prime}$ is the edge following $e$ in the counterclockwise order at $e^{-}$. Given a smooth embedding of a map $M$, for each corner of $M$, we let ang $\left(e, e^{\prime}\right)$ be the angle between $e$ and $e^{\prime}$ at $v$. In a smooth embedding, every oriented edge $e$ of the map has a well defined total geodesic curvature in the embedding, which we denote $\kappa_{g}(e)$.

A smooth Riemannian surface with conical singularities has a Gaussian curvature $\kappa$ associated with its metric, which is a signed measure on the surface (see e.g. [3, Chapter 5]). The curvature measure $\kappa$ is absolutely continuous with respect to the area measure on the surface except for possible atoms at the conical singularities at the vertices. We denote by $\kappa_{s}(v)$ be the atom of curvature at a vertex $v$, and by $\kappa(f)$ be the total curvature of the face $f$, which is well-defined if $f$ has finite degree. In a smooth embedding of a map, the mass of the atom of curvature at a vertex is given by

$$
\kappa_{s}(v)=2 \pi-\sum_{e: e^{-}=v} \operatorname{ang}(e) .
$$

Given a smooth embedding of a map with a locally finite dual, we define the total curvature at a vertex $v$ by

$$
\kappa(v)=\kappa_{s}(v)+\sum_{f \perp v} \frac{\kappa(f)}{\operatorname{deg}(f)} .
$$

We are now ready to state our "invariance of the average curvature" theorem. 
Theorem 4.10. Let $(M, \rho)$ be a unimodular random rooted map with locally finite dual and let $(S, z)$ be a smooth unimodular embedding of $M$. Suppose further that either

(1) $\kappa_{g}(e)=0$ for every oriented edge $e$ of $M$, and $\kappa(f) \leq 0$ for every face $f$ of $M$, or

(2) $\sum_{e: e^{-}=\rho}\left|\kappa_{g}(e)\right|$ and $|\kappa(\rho)|$ both have finite expectation. Then

$$
\mathbb{E}[\kappa(\rho)]=\mathbb{K}(M, \rho) .
$$

We remark that it is possible to use Theorem 4.10 to prove Proposition 4.5 by embedding $M$ in the polygonal manifold associated to $\omega$. We have restricted Theorem 4.10 to maps with locally finite duals mainly for the sake of brevity. It is possible to generalize this identity to maps with infinite faces under suitable integrability assumptions.

The necessity of some integrability condition is demonstrated by the following example. Consider the map obtained by a unimodular triangulation of each of the two infinite faces of $\mathbb{Z}$ (e.g. as in Figure 5). This map admits a unimodular embedding in either the Euclidean or hyperbolic plane, by drawing the edges of $\mathbb{Z}$ as segments of length one along a bi-infinite geodesic, and drawing the other edges of the map as semi-circular arcs. This embedding is a measurable function of the map, and is therefore unimodular. There are no conical singularities in either case, but $\sum_{f \perp v} \kappa(f) / \operatorname{deg}(f)$ is 0 in the Euclidean embedding and negative at every vertex in the hyperbolic embedding, violating invariance of the curvature. In fact, it is easily seen that the map has average degree 6 , so that $\mathbb{K}(M, \rho) \geq 0$. It is also easy to see that the hyperbolic embedding does not satisfy the integrability requirements of Theorem 4.10.

Proof. The proof relies on a generalization of the mass transport that we used to prove Proposition 4.5 in the case that both duals were locally finite. Now, however, we need to allow for the curvature of the faces and the geodesic curvature of the edges, using the fact that the angle sum around a face is related to these quantities by the Gauss-Bonnet Theorem (see e.g. [3, Chapter VI, Section 7]).

Each corner $\left(e, e^{\prime}\right)$ of $M$ is associated with a vertex $v=e^{-}$and face $f=e^{r}$. The Gauss-Bonnet Theorem implies that for any face $f$ of $M$,

$$
\sum_{e: e^{r}=f}\left[\pi-\operatorname{ang}\left(e, e^{\prime}\right)\right]+\kappa(f)+\sum_{e: e^{r}=f} \kappa_{g}(e)=2 \pi .
$$

Here, the sum over edges is taken for the directed clockwise cycle around $f$. We rewrite this identity as

$$
\sum_{e: e^{r}=f}\left[\operatorname{ang}\left(e, e^{\prime}\right)-\frac{\kappa(f)}{\operatorname{deg}(f)}-\kappa_{g}(e)\right]=(\operatorname{deg}(f)-2) \pi .
$$

We now define two mass transports on $M$. First, for each directed edge $e=(u, v)$, let $u$ send to $v$ a mass of $\kappa_{g}(e)$. The expected mass sent from $\rho$ is $\mathbb{E} \sum_{e^{-}=\rho} \kappa_{g}(e)$, while the expected mass received by $\rho$ is $\mathbb{E} \sum_{e^{-}=\rho} \kappa_{g}(-e)=-\mathbb{E} \sum_{e^{-}=\rho} \kappa_{g}(e)$. Equating the two we find that

$$
\mathbb{E} \sum_{e^{-}=\rho} \kappa_{g}(e)=0
$$

The integrability condition needed to apply the signed Mass-Transport Principle here is given by condition (2), while the equality is trivial under condition (1).

Our second mass transport is defined in two stages as follows. For each vertex $u$ and corner $\left(e, e^{\prime}\right)$ at $u$ with $e^{r}=f$, the vertex $u$ sends to the face $f$ a mass of $\operatorname{ang}(e)-\frac{\kappa(f)}{\operatorname{deg}(f)}-\kappa_{g}(e)$. 
Each face then redistributes the mass it recieves uniformly to the vertices at its corners, with the usual multiplicity. This defines a mass transport $\phi$, given explicitly by

$$
\phi(u, v)=\sum_{f: f \perp u, f \perp v} \sum_{e: e^{-}=v, e^{r}=f} \frac{1}{\operatorname{deg}(f)}\left[\operatorname{ang}\left(e, e^{\prime}\right)-\frac{\kappa(f)}{\operatorname{deg}(f)}-\kappa_{g}(e)\right] .
$$

Summing the mass sent from $\rho$ through a face $f$ to the corners of $f$ gives

$$
\sum_{v} \phi(\rho, v)=\sum_{e^{-}=\rho} \operatorname{ang}\left(e, e^{\prime}\right)-\frac{\kappa(f)}{\operatorname{deg}(f)}-\kappa_{g}(e) .
$$

If (1) holds then $\phi(u, v)$ is non-negative for every $u$ and $v$, while if (2) holds then the expectation $\mathbb{E} \sum_{v \in V}|\phi(\rho, v)|$ is finite, and we can apply the Mass-Transport Principle to $\phi$ in either case. Taking expectations and using (4.3), we have

$$
\mathbb{E} \sum_{v} \phi(\rho, v)=\mathbb{E}\left[\theta(\rho)-\sum_{e^{-}=\rho} \frac{\kappa(f)}{\operatorname{deg}(f)}\right]=2 \pi-\mathbb{E} \kappa(\rho) .
$$

Applying (4.2), it follows that the total mass passing through a face $f$ is $(\operatorname{deg}(f)-2) \pi$, and so the expected total mass received by $\rho$ is

$$
\mathbb{E} \sum_{v} \phi(v, \rho)=\mathbb{E} \sum_{f \perp \rho} \frac{(\operatorname{deg}(f)-2) \pi}{\operatorname{deg}(f)}=2 \pi-\mathbb{K}(M, \rho),
$$

completing the proof.

Example 4.11 (Voronoi diagrams and Delaunay triangulations). We say that a set of points in the plane is in general position if no four points in the set lie on any given circle or line. Given a set $Z$ of points in general position in either the Euclidean plane or the hyperbolic plane, the Delaunay triangulation of $Z$ is a simple triangulation with vertex set $Z$, defined so that three points $u, v$, and $w$ of $Z$ form a triangle if and only if the unique disc containing $u, v$, and $w$ in its boundary does not contain any other points of $Z$.

Suppose that $Z$ is an isometry-invariant, locally finite point process in either the Euclidean plane or the hyperbolic plane, and let $\hat{Z}$ be the Palm version of $Z$ that is conditioned to have a point at the origin. Then the Delaunay triangulation of $\hat{Z}$, rooted at 0 is unimodular. See [16] for a study of the Poissonian case. This triangulation, embedded with geodesic segments for edges, satisfies condition (1) of Theorem 4.10, and we deduce that, unsurprisingly, Delaunay triangulations of hyperbolic point processes are hyperbolic while Delaunay triangulations of Euclidean point processes are parabolic. (This also follows from the methods of [4].) The dual of the Delaunay triangulation is the Voronoi diagram of the point process, which can also be made unimodular as in Section 2.5. The Voronoi diagram is also hyperbolic in the hyperbolic plane and parabolic in the Euclidean plane.

\section{$5 \quad$ Spanning forests}

It is evident from Figure 1 that the analysis of random spanning forests in a map $M$ is central to the proof of Theorem 1. The free and wired uniform spanning forests FUSF and WUSF are known to encode properties of the random walk: any graph $G$ has the intersection property if and only if its wired uniform spanning forest is almost surely connected, while $G$ admits 
non-constant harmonic functions of finite Dirichlet energy if and only if the laws of the free and wired uniform spanning forest are distinct. The (free and wired) minimal spanning forests, on the other hand, are related to Bernoulli bond percolation: $p_{c}(G)<p_{u}(G)$ if and only if the laws of the two minimal spanning forests are distinct.

\subsection{Uniform Spanning Forests}

Our primary method to relate the average curvature of a map $M$ with the various probabilistic properties listed in Theorem 1 is a formula relating the average degree of the free uniform spanning forest of $M$ to its average curvature. To this aim, we begin with a succinct review of uniform spanning forest. We refer the reader to $[13,60]$ for a comprehensive treatment.

For a finite graph $G$, let UST $G$ be the uniform measure on spanning trees of $G$ (i.e. connected subgraphs of $G$ containing every vertex and no cycles), which is the law of a percolation on $G$. There are two natural ways to define infinite volume limits of the uniform spanning tree. Let $G=(V, E)$ be an infinite, locally finite, connected graph. An exhaustion of $G$ is an increasing sequence $\left\langle V_{n}\right\rangle_{n \geq 1}$ of finite connected subsets of $V$ such that $\bigcup_{n \geq 1} V_{n}=V$. Given an exhaustion $\left\langle V_{n}\right\rangle_{n \geq 1}$ of $G$, we define $G_{n}$ to be the subgraph of $G$ induced by $V_{n}$ for each $n \geq 1$. The free uniform spanning forest measure of $G$ is defined as the weak limit of the uniform spanning tree measures on the graphs $G_{n}$. That is, for each finite set $S \subset E$,

$$
\operatorname{FUSF}_{G}(S \subset T):=\lim _{n \rightarrow \infty} \operatorname{UST}_{G_{n}}(S \subset T) .
$$

For each $n \geq 1$, we also construct a graph $G_{n}^{*}$ from $G$ by identifying every vertex in $V \backslash V_{n}$ into a single vertex $\partial_{n}$, and deleting all of the resulting self loops from $\partial_{n}$ to itself. We then define the wired uniform spanning forest measure of $G$ to be the weak limit of the uniform spanning tree measures on the graphs $G_{n}^{*}$. That is, for each finite set $S \subset E$,

$$
\operatorname{WUSF}_{G}(S \subset T):=\lim _{n \rightarrow \infty} \operatorname{UST}_{G_{n}^{*}}(S \subset T) .
$$

The study of uniform spanning forests was pioneered by Pemantle [64], who showed that that both limits exist for any graph $G$ and in particular are independent of the choice of exhaustion.

A crucial link between the USFs and amenability is the following result of Aldous and Lyons [2, Proposition 18.14].

Theorem 5.1 (Theorem 1, (2) implies (12)). If $(G, \rho)$ is an invariantly amenable unimodular random rooted graph, then $\mathrm{FUSF}_{G}=\mathrm{WUSF}_{G}$ almost surely.

The free and wired uniform spanning forests enjoy the following properties:

Free dominates wired: The measure $\mathrm{FUSF}_{G}$ stochastically dominates the measure WUSF ${ }_{G}$ for every graph $G$.

Domination and subgraphs: Let $H$ be a connected subgraph of $G$. Then the FUSF of $H$ stochastically dominates the restriction of the FUSF of $G$ to $H$.

Expected degree of the WUSF: The expected degree in the WUSF of the root of any unimodular random rooted graph is 2 [2, Proposition 7.3].

Note that, since a connected spanning forest cannot be strictly contained in another spanning forest, the stochastic domination above has the following immediate consequence. 
Proposition 5.2 (Theorem 1, (13) implies (12)). Let $G$ be a graph. If the wired uniform spanning forest of $G$ is connected almost surely, then the wired and free spanning forests of $G$ coincide.

Let $(G, \rho)$ be a unimodular random rooted graph and $\mathfrak{F}$ a sample of either WUSF $_{G}$ or $\operatorname{FUSF}_{G}$. If $\mathfrak{F}$ is interpreted as a percolation on $G$, then the marked graph $(G, \rho, \mathfrak{F})$ is also unimodular. Indeed, since the definitions of $\mathrm{FUSF}_{G}$ and $\mathrm{WUSF}_{G}$ do not depend on the choice of exhaustion, for each mass transport $f: \mathcal{G}_{\bullet \bullet}^{\{0,1\}} \rightarrow[0, \infty]$, the expectations

$$
f^{F}(G, u, v)=\operatorname{FUSF}_{G}[f(G, u, v, \mathfrak{F})] \quad \text { and } \quad f^{W}(G, u, v)=\operatorname{WUSF}_{G}[f(G, u, v, \mathfrak{F})]
$$

are also measurable. Using this observation, we deduce the mass-transport principle for $(G, \rho, \mathfrak{F})$ from that of $(G, \rho)$.

Connections to random walk and potential theory. Although the uniform spanning tree of each $G_{n}$ or $G_{n}^{*}$ is connected, the limiting random subgraph can be disconnected. Indeed, Pemantle [64] proved that WUSF and FUSF of $\mathbb{Z}^{d}$ coincide for all $d \geq 1$, and are connected if and only if $d \leq 4$. A complete characterisation of the connectivity of the WUSF was given by Benjamini, Lyons, Peres and Schramm [13]. A connected, locally finite graph is said to have the intersection property if the traces of two independent simple random walks started from any two vertices of the graph have infinite intersection almost surely (or, equivalently, if the two traces intersect almost surely). A graph is said to have the non-intersection property if the traces of two independent simple random walks on the graph have finite intersection almost surely.

Theorem 5.3 ([13]: Theorem 1, equivalence of (14) and (13)). Let $G$ be an infinite, locally finite, connected graph and let $\mathfrak{F}$ be a sample of $\mathrm{WUSF}_{G}$. Then $\mathfrak{F}$ is connected almost surely if and only if $G$ has the intersection property. Moreover, if $G$ has the non-intersection property, then $\mathfrak{F}$ has infinitely many connected components almost surely.

In general, a graph may have neither of the intersection and non-intersection properties. For example, the graph formed by connecting two disjoint copies of $\mathbb{Z}^{3}$ by a single edge between their origins does not have either property. However, it is easily seen that this is not the case for reversible random rooted graphs.

Lemma 5.4. Let $(G, \rho)$ be a unimodular random rooted graph with $\mathbb{E}[\operatorname{deg}(\rho)]<\infty$. Then almost surely $G$ has either the intersection property or the non-intersection property. Consequently, the wired uniform spanning forest of a unimodular random rooted graph with finite expected degree is almost surely either connected or has infinitely many connected components.

Proof. By biasing by the degree we may assume that $(G, \rho)$ is reversible. We may assume also that $(G, \rho)$ is ergodic, otherwise we take an ergodic decomposition. Let $\left(X_{n}\right)_{n \geq 0}$ and $\left(X_{-n}\right)_{n \geq 0}$ be independent random walks on $G$ started at $\rho$. Then the event that the traces of $\left(X_{n}\right)_{n \geq 0}$ and $\left(X_{-n}\right)_{n \geq 0}$ have infinite intersection is an invariant event for the stationary sequence $\left(G,\left(X_{n+k}\right)_{n \in \mathbb{Z}}\right)_{k \in \mathbb{Z}}$ and therefore has probability either zero or one by ergodicity (see $[2, \S 4]$ and $[4$, Theorem 3.1]).

Recall that a function $h: V \rightarrow \mathbb{R}$ defined on the vertex set of a graph $G=(V, E)$ is said to be harmonic if

$$
h(v)=\frac{1}{\operatorname{deg}(v)} \sum_{u \sim v} h(u)
$$


for every vertex $v$ of $G$, or equivalently if $\left(h\left(X_{n}\right)\right)_{n \geq 0}$ is a martingale when $\left(X_{n}\right)_{n \geq 0}$ is a random walk on $G$. A graph is said to be Liouville if it does not admit any non-constant bounded harmonic functions, and non-Liouville otherwise. The following well-known proposition follows from the martingale convergence theorem, see [60, Exercise 14.28].

Proposition 5.5 (Theorem 1, (14) implies (10)). Let $G$ be a connected graph. If $G$ has the intersection property, then $G$ is Liouville.

The converse of Proposition 5.5 does not hold for general graphs. For example, $\mathbb{Z}^{d}$ is Liouville for all $d \geq 1$ but has the intersection property only for $d \leq 4$. However, Benjamini, Curien and Georgakopoulos [10] proved that the converse does hold for planar graphs.

Theorem 5.6 ([10]; Theorem 1, (10) implies (14)). Let $G$ be a planar graph. Then $G$ is Liouville if and only if it has the intersection property.

The Dirichlet energy of a function $f: V \rightarrow \mathbb{R}$ defined on the vertex set of $G$ is defined to be

$$
\mathcal{E}(f)=\frac{1}{2} \sum_{e \in E \rightarrow}\left(f\left(e^{-}\right)-f\left(e^{+}\right)\right)^{2} .
$$

The following is classical; see [60, Exercise 9.43].

Proposition 5.7 (Theorem 1, (10) implies (11)). Let $G$ be a connected graph. Then the bounded harmonic functions of finite Dirichlet energy are dense in the space of harmonic functions of finite Dirichlet energy with norm $\|f\|^{2}=\mathcal{E}(f)$. In particular, if $G$ admits a nonconstant harmonic function of finite Dirichlet energy, then $G$ admits a bounded non-constant harmonic function of finite Dirichlet energy.

Benjamini, Lyons, Peres and Schramm [13] also relate analytic properties of $G$ to the WUSF and FUSF of $G$.

Theorem 5.8 ([13]; Theorem 1, equivalence of items (12) (11)). Let $G$ be an infinite connected graph. Then the measures $\mathrm{FUSF}_{G}$ and $\mathrm{WUSF}_{G}$ are distinct if and only if $G$ admits non-constant harmonic functions of finite Dirichlet energy.

In general, the FUSF is far less understood as the WUSF: no criterion for its connectivity is known, nor is it known whether the number of components of the FUSF is non-random in every graph. However, for simply connected planar maps, the FUSF is relatively well understood thanks to the following duality: Given a map $M$ and a set $W \subset E$, let $W^{\dagger}:=$ $\left\{e^{\dagger} \in E^{\dagger}: e \notin W\right\}$ be the set of dual edges whose corresponding primal edges are not contained in $W$. Observe that if $t$ is a spanning tree of a finite planar map $M$, then the dual $t^{\dagger}$ is a spanning tree of $M^{\dagger}$ - it is connected because $t$ has no cycles, and has no cycles because $t$ is connected. This observation leads to the following.

Proposition 5.9 (USF Duality [13, Theorem 12.2]). Let $M$ be a simply connected map with locally finite dual $M^{\dagger}$ and let $\mathfrak{F}$ be a random variable with law $\mathrm{FUSF}_{M}$. Then $\mathfrak{F}^{\dagger}$ has law WUSF $_{M^{\dagger}}$.

In general, if $M$ is an infinite simply connected map and $\mathfrak{F}$ is an essential spanning forest of $M$ (that is, a spanning forest such that every component is infinite), then $\mathfrak{F}^{\dagger}$ is an essential spanning forest of $M^{\dagger}$ - it is a forest because every component of $\mathfrak{F}$ is infinite, and is essential because $\mathfrak{F}$ has no cycles. Moreover, the forest $\mathfrak{F}$ is connected if and only if every component 
of $\mathfrak{F}^{\dagger}$ is one-ended. Furthermore, a combination of results from Aldous and Lyons [2] yields the following.

Proposition 5.10 (Theorem 1, (13) implies (2)). Let $(G, \rho)$ be a unimodular random rooted graph with $\mathbb{E}[\operatorname{deg}(\rho)]<\infty$ and let $\mathfrak{F}$ be a sample of $\mathrm{WUSF}_{G}$. If $\mathfrak{F}$ is connected almost surely, then $(G, \rho)$ is invariantly amenable.

Proof. By Proposition 7.3 of [2], the average degree of $\mathfrak{F}$ is 2 . By Theorem 6.2 there, this implies that $\mathfrak{F}$ has at most two ends. Since $\mathfrak{F}$ is connected, Theorem 8.9 of [2] implies that $(G, \rho)$ is invariantly amenable.

\subsection{Connectivity and degrees in the FUSF}

The first main result of this section relates the average curvature and the expected degree of the FUSF in a simply connected unimodular random rooted map.

Theorem 5.11. Let $(M, \rho)$ be an infinite, simply connected unimodular random map. Suppose that $\mathbb{E}[\operatorname{deg}(\rho)]<\infty$, and let $\mathfrak{F}$ be a sample of $\mathrm{FUSF}_{M}$. Then

$$
\mathbb{E}\left[\operatorname{deg}_{\widetilde{F}}(\rho)\right]=\frac{1}{\pi} \mathbb{E}[\theta(\rho)]=2-\frac{1}{\pi} \mathbb{K}(M, \rho) .
$$

As an easy consequence we get the following component of Theorem 1.

Corollary 5.12 (Theorem 1 , equivalence of (1) and (12) and $\mathbb{K} \leq 0)$. Let $(M, \rho)$ be an infinite, simply connected unimodular random map with $\mathbb{E}[\operatorname{deg}(\rho)]<\infty$. Then the average curvature satisfies $\mathbb{K}(M, \rho) \leq 0$, and equals zero if and only if $\mathrm{FUSF}_{M}=\mathrm{WUSF}_{M}$ almost surely.

Proof. Let $\mathfrak{F}$ be a sample of $\mathrm{FUSF}_{M}$. Since the expected degree of the WUSF in any unimodular random rooted graph is two, and $\mathrm{FUSF}_{M}$ stochastically dominates $\mathrm{WUSF}_{M}$, we have that $\mathbb{E}\left[\operatorname{deg}_{\mathfrak{F}}(\rho)\right] \geq 2$ and that the measures $\operatorname{FUSF}_{M}$ and $\operatorname{WUSF}_{M}$ differ almost surely if and only if this inequality is strict. We conclude by applying Theorem 5.11.

Theorem 5.11 follows as an immediate corollary of Proposition 4.5 and the following theorem, which is the second main result of this section. In Section 5.5, we give an alternative, duality-based proof of Theorem 5.11 that does not rely on Theorem 5.13 or Proposition 4.5, and also applies to the free minimal spanning forest.

Theorem 5.13 (Connectivity of the FUSF). Let $(M, \rho)$ be an infinite, simply connected unimodular random rooted map with $\mathbb{E}[\operatorname{deg}(\rho)]<\infty$. Then the free uniform spanning forest of $M$ is connected almost surely.

Theorem 5.13 is complemented by work by the second and third authors [48], who prove the corresponding theorem for all simply connected planar maps with bounded degrees. Since the measure $\mathrm{FUSF}_{G}$ stochastically dominates $\mathrm{WUSF}_{G}$ for every graph $G$, and has no cycles, we deduce the following immediate corollary.

Corollary 5.14 (Theorem 1, equivalence of (12) and (13)). Let $(M, \rho)$ be an infinite, simply connected unimodular random map with $\mathbb{E}[\operatorname{deg}(\rho)]<\infty$. Then $\mathrm{FUSF}_{M}=\mathrm{WUSF}_{M}$ if and only if the wired uniform spanning forest of $M$ is connected almost surely. 

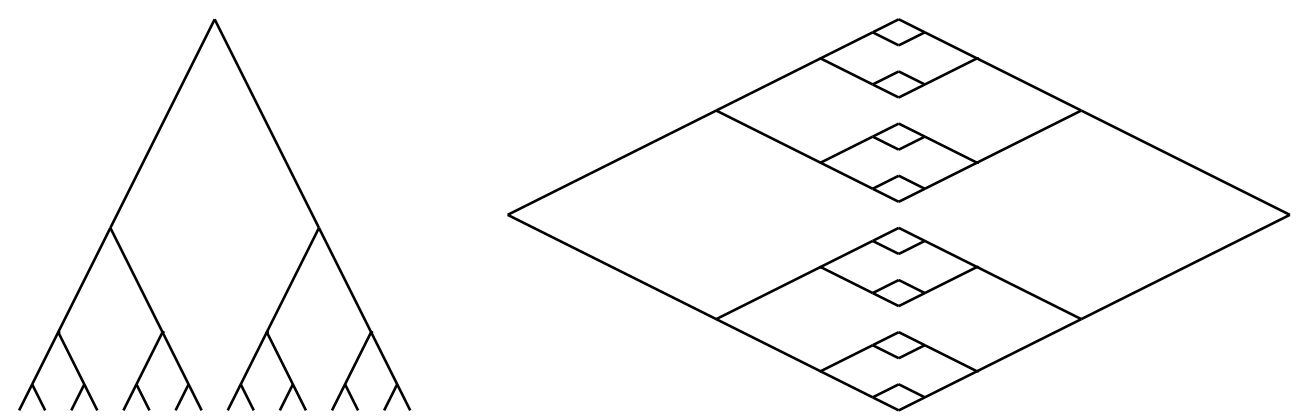

Figure 6. The maps $T_{4}$ (left) and $M_{4}$ (right).

Having finite expected degree is necessary for this to hold. Let $T_{n}$ be a binary tree of height $n$ drawn in the plane. The Benjamini-Schramm limit of $T_{n}$ as $n$ tends to infinity is known as the canopy tree, and can be thought of as an 'infinite binary tree viewed from a leaf'. Let $M_{n}$ be the finite map obtained by drawing two copies of $T_{n}$ so that one is the reflection of the other, and attaching these two copies together at their leaves (see Figure 6). Replace each edge of $M_{n}$ at distance $k$ from the leaves by $3^{k}$ parallel edges, and call the resulting map $M_{n}^{\prime}$. The Benjamini-Schramm limit $\left(M^{\prime}, \rho\right)=\lim M_{n}^{\prime}$ exists and is formed of two similarly thickened canopy trees, attached together at their leaves.

The random walk on $M^{\prime}$ has constant drift away from the leaves, so it is possible for two random walks to be absorbed in different halves of $M^{\prime}$. Thus $M^{\prime}$ does not have the intersection property (nor the non-intersection property), and WUFF $_{M^{\prime}}$ is not connected. However, the space of bounded harmonic functions is spanned by the probability of being absorbed at either side of $M^{\prime}$. It follows that bounded (and hence all) harmonic functions have infinite Dirichlet energy, and that $\mathrm{FUSF}_{M^{\prime}}=\mathrm{WUSF}_{M^{\prime}}$.

Towards a proof of Theorem 5.13, observe that if $M$ has locally finite dual almost surely, then, by Proposition 5.9, it suffices to showt that every component of the WUSF of $M^{\dagger}$ is one-ended almost surely. Fortunately, this is known to hold in several large classes of graphs: The following was proven by the second author $[47,46]$. See also earlier works by Pemantle [64], Benjamini, Lyons, Peres, and Schramm [13], and Aldous and Lyons [2]. See [59] for further one-endedness results in the deterministic setting.

Theorem $5.15([47,46])$. Let $(G, \rho)$ be a transient unimodular random rooted graph. Then every component of the wired uniform spanning forest of $G$ is one-ended almost surely.

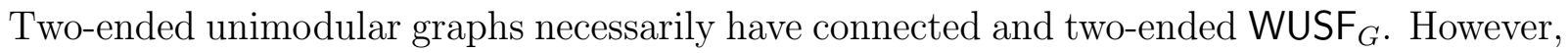
it is an open question whether WUSF components in a one-ended, recurrent, unimodular graphs can have two ends (more than two is impossible). The final result of this section settles the planar case.

Theorem 5.16. Let $(M, \rho)$ be a recurrent unimodular random rooted planar map with $\mathbb{E}[\operatorname{deg}(\rho)]<\infty$. Then the wired uniform spanning forest of $M$ has the same number of ends as $M$ almost surely (which is either one or two since $M$ is recurrent).

\subsection{Proof of Theorems 5.13 and 5.16}

The proofs are closely linked and are split according to whether the dual map $M^{\dagger}$ is locally finite or not. 
Proofs of when $M^{\dagger}$ is locally finite. Let $(M, \rho)$ be a unimodular random rooted map with $\mathbb{P}[\operatorname{deg}(\rho)]<\infty$, and suppose that the dual $M^{\dagger}$ is locally finite almost surely. We may assume that $(M, \rho)$ is ergodic, otherwise we take an ergodic decomposition. Let $\mathfrak{F}$ be a sample of FUSF $_{M}$ and let $\mathfrak{F}^{\dagger}$ be the dual forest. Since $\mathbb{E}[\operatorname{deg}(\rho)]<\infty$, the law of $\left(M^{\dagger}, \rho^{\dagger}\right)$ is absolutely continuous w.r.t. a reversible random rooted map by Proposition 2.4.

If $M^{\dagger}$ is almost surely transient, Theorem 5.15 implies that every component of $\mathfrak{F}^{\dagger}$ is one-ended almost surely, and we deduce that $\mathfrak{F}$ is connected almost surely.

If $M^{\dagger}$ is almost surely recurrent, then $\mathfrak{F}^{\dagger}$ is connected almost surely. Being unimodular and recurrent, this tree has at most two ends. Since $M^{\dagger}$ is locally finite, $M$ and $M^{\dagger}$ have the same number of ends. If $\mathfrak{F}^{\dagger}$ was two-ended, while $M$ is one-ended, then $\mathfrak{F}$ would have exactly two components, contradicting Lemma 5.4. If $M$ is two-ended, then so is the spanning tree $\mathfrak{F}$, giving Theorem 5.16. In either case we deduce that $\mathfrak{F}$ is connected almost surely.

The remainder of this section is dedicated to the proof of Theorems 5.13 and 5.16 in the presence of infinite faces. We begin by developing a variant of Wilson's algorithm that allows us to sample the dual of the FUSF using random walks when the dual is not locally finite.

Given a graph $G$ and a path $\gamma$ in $G$ that is either finite or transient, i.e. visits each vertex of $G$ at most finitely many times, the loop-erasure $\operatorname{LE}(\gamma)$ is formed by erasing cycles from $\gamma$ chronologically as they are created. Formally, $\operatorname{LE}(\gamma)_{i}=\gamma_{t_{i}}$ where the times $t_{i}$ are defined recursively by $t_{0}=0$ and $t_{i}=1+\max \left\{t \geq t_{i-1}: \gamma_{t}=\gamma_{t_{i-1}}\right\}$. If the graph has multiple edges, then $\operatorname{LE}(\gamma)$ uses the edge $\gamma$ uses between times $t_{i}-1$ and $t_{i}$. The loop-erasure of a simple random walk is known as loop-erased random walk, was introduced by Lawler [57], and was extensively studied.

Wilson's algorithm [67] is a method of sampling a uniform spanning tree of a finite graph by joining together loop-erased random walk paths. Benjamini, Lyons, Peres and Schramm [13] introduced a variant of Wilson's algorithm for sampling the WUSF of an infinite transient graph, known as Wilson's algorithm rooted at infinity. Let $G=(V, E)$ be a connected, locally finite graph and let $\left(v_{i}\right)_{i \geq 1}$ be an enumeration of the vertex set $V$. We sample a sequence of forests $\left(\mathfrak{F}_{i}\right)_{i \geq 0}$ in $G$ recursively as follows:

(1) If $G$ is finite or recurrent, fix a vertex $v_{0}$ of $G$ and let $\mathfrak{F}_{0}=\left\{v_{0}\right\}$. If $G$ is transient set $\mathfrak{F}_{0}=\emptyset$.

(2) Given $\mathfrak{F}_{i}$, start a simple random walk from $v_{i}$ in $M$, independent of everything already sampled, and stopped if and when it first hits a vertex already included in $\mathfrak{F}_{i}$.

(3) Take the loop-erasure of this random walk path, and let $\mathfrak{F}_{i+1}$ be the union of $\mathfrak{F}_{i}$ and this loop-erased path.

(4) Let $\mathfrak{F}=\bigcup_{i \geq 0} \mathfrak{F}_{i}$.

The resulting random forest has law UST $_{G}$ when $G$ is finite [67] and WUSF $_{G}$ when $G$ is infinite [13].

If $M^{\dagger}$ is locally finite, then we can sample FUSF $_{M}$ by using Wilson's algorithm to sample $\mathfrak{F}^{\dagger}$ with law WUSF $_{M^{\dagger}}$. We introduce a variation for sampling the dual of FUSF $_{M}$, when $M^{\dagger}$ is not locally finite. Let $F_{\text {fin }}$ and $F_{\infty}$ be respectively the set of finite and infinite faces of $M$. The following is called Wilson's algorithm rooted at $\{\infty\} \cup F_{\infty}$.

Let $\left(f_{i}\right)_{i \geq 1}$ be an enumeration of the finite faces of $M$. We sample an increasing sequence of forests $\left(\mathfrak{F}_{i}^{\dagger}\right)_{i \geq 0}$ in $M^{\dagger}$ recursively as follows:

$\left(1^{\prime}\right)$ Let $\mathfrak{F}_{0}^{\dagger}=F_{\infty}$. 
$\left(2^{\prime}\right)$ Given $\mathfrak{F}_{i}^{\dagger}$, start a simple random walk from $f_{i}$ in $M^{\dagger}$, independent of everything we have already sampled, and stopped if and when it first hits a face already included in $\mathfrak{F}_{i}^{\dagger}$. In particular, the walk is stopped if it hits a face of infinite degree, so that it is well-defined.

$\left(3^{\prime}\right)$ Take the loop-erasure of this random walk path, and let $\mathfrak{F}_{i+1}^{\dagger}$ be the union of $\mathfrak{F}_{i}^{\dagger}$ and this loop-erased path.

$\left(4^{\prime}\right)$ Let $\mathfrak{F}^{\dagger}=\bigcup_{i \geq 0} \mathfrak{F}_{i}^{\dagger}$.

Proposition 5.17. Let $M=(V, E, \sigma)$ be a simply connected map with dual $M^{\dagger}$ and let $\mathfrak{F}^{\dagger}$ be a random subset of $E^{\dagger}$ sampled by Wilson's algorithm rooted at $\{\infty\} \cup F_{\infty}$. Then $\mathfrak{F}=\left(\mathfrak{F}^{\dagger}\right)^{\dagger}$ is a sample of $\mathrm{FUSF}_{M}$.

Proof. Let $\left(V_{n}\right)_{n \geq 0}$ be an exhaustion of $V$ such that the submap of $M$ induced by $V \backslash V_{n}$, denoted $M_{n}$, does not have any finite connected components for any $n$. The dual of $M_{n}$ may be constructed from $M^{\dagger}$ by identifying every face $f$ of $M$ that does not have all of its vertices included in $V_{n}$ into a single vertex $\partial_{n}$, and deleting all the self-loops that are created. In particular, all infinite faces of $M$ are identified into $\partial_{n}$ for every $n \geq 1$.

Note that a simple random walk $\left(X_{n}\right)_{n \geq 0}$ on $M^{\dagger}$, started at some finite face $f$ and stopped if and when it hits $F_{\infty}$, is either transient or killed at a finite time almost surely. Given these observations, the rest of the proof, below, proceeds similarly to the usual proof of the veracity of Wilson's algorithm rooted at infinity [13, Theorem 5.1].

Let $H$ be a finite set of edges of $M^{\dagger}$, and let $f_{1}, \ldots, f_{l}$ be an enumeration of the set of faces $f$ of $M$ that are endpoints of at least one of the edges in $H$. Let $\left(\left(X_{j}^{i}\right)_{j \geq 0}: i=1, \ldots, l\right)$ be a collection of independent random walks in $M^{\dagger}$, where the walk $\left(X_{j}^{i}\right)_{j \geq 0}$ is started at $f_{i}$ and stopped the first time that it hits an infinite face of $M$. Run Wilson's algorithm in $M_{n}^{\dagger}$, rooted at $\partial_{n}$ and starting with the faces $f_{1}, \ldots, f_{l}$ in that order, using the random walks $\left(X_{j}^{i}\right)_{j \geq 0}$ : For each $i \in[l]$, let $\tau_{i}^{n}$ be the first time that the random walk $\left(X_{j}^{i}\right)_{j \geq 0}$ visits the portion of the spanning tree generated up to time $i-1$, so that

$$
\mathrm{UST}_{M_{n}^{\dagger}}(H \subset T)=\mathbb{P}\left(H \subseteq \bigcup_{i \leq l} \operatorname{LE}\left(\left(X_{j}^{i}\right)_{j=0}^{\tau_{i}^{n}}\right)\right) .
$$

Now, similarly, run Wilson's algorithm on $M^{\dagger}$ rooted at $\{\infty\} \cup F_{\infty}$, starting with the faces $f_{1}, \ldots, f_{l}$ in that order and using the random walks $\left(X_{j}^{i}\right)_{j \geq 0}$, and let $\tau_{i}$ be the first time that the random walk $\left(X_{j}^{i}\right)_{j \geq 0}$ visits the portion of the spanning tree generated up to time $i-1$ (which might now be infinite). Since the walks $\left(X_{j}^{i}\right)_{j \geq 0}$ are finite or transient almost surely, we have that

$$
\tau_{i}^{n} \rightarrow \tau_{i} \quad \text { and } \quad \operatorname{LE}\left(\left(X_{j}^{i}\right)_{j=0}^{\tau_{i}^{n}}\right) \rightarrow \operatorname{LE}\left(\left(X_{j}^{i}\right)_{j=0}^{\tau_{i}}\right)
$$

almost surely as $n \rightarrow \infty$. It follows that

$$
\begin{aligned}
\operatorname{FUSF}_{M}\left(H \subset \mathfrak{F}^{\dagger}\right) & =\lim _{n \rightarrow \infty} \operatorname{UST}_{M^{\dagger}}(H \subset T)=\lim _{n \rightarrow \infty} \mathbb{P}\left(H \subseteq \bigcup_{i \leq l} \operatorname{LE}\left(\left(X_{j}^{i}\right)_{j=0}^{\tau_{i}^{n}}\right)\right) \\
& =\mathbb{P}\left(H \subseteq \bigcup_{i \leq l} \operatorname{LE}\left(\left(X_{j}^{i}\right)_{j=0}^{\tau_{i}}\right)\right)
\end{aligned}
$$

completing the proof.

Proposition 5.18. Let $(M, \rho)$ be a simply connected, unimodular random rooted map with $\mathbb{P}[\operatorname{deg}(\rho)]<\infty$ and suppose that the dual $M^{\dagger}$ contains a vertex of infinite degree almost surely. 
Let $\mathfrak{F}$ be a sample of $\mathrm{FUSF}_{M}$. Then every connected component of $\mathfrak{F}^{\dagger} \backslash F_{\infty}$ is finite almost surely, and consequently $\mathfrak{F}$ is connected almost surely.

Proof of Proposition 5.18. The main ingredient is the observation that a random walk on $M^{\dagger}$ started at a finite face will hit $F_{\infty}$ in a finite time almost surely.

To see this, we first bias the law of $M$ by $\operatorname{deg}(\rho)$ to get a reversible map. Next, we convert $M^{\dagger}$ into a a (possibly disconnected) graph $G^{\prime}$ with edge set $E^{\dagger}$ as follows. For each edge $e^{\dagger} \in E^{\dagger}$ with an endpoint in $F_{\infty}$, replace this endpoint with a new vertex of degree one. The vertex set of $G^{\prime}$ is $F_{\text {fin }} \cup L$, where $L$ is the set of new degree one vertices of $G^{\prime}$ corresponding to edges of $M$ incident to $F_{\infty}$. We define a marking $m$ on the vertices of $G^{\prime}$ where $m(v)=\mathbb{1}(v \in L)$. Let $\eta$ be chosen uniformly from among the oriented edges emanating from $\rho$, and let $\rho^{\prime}$ be the tail of $\eta^{\dagger}$ in $G^{\prime}$. Let $G^{\prime \prime}$ denote the connected component of $G^{\prime}$ containing $\rho^{\prime}$. A similar argument to that used in Section 2.5 shows that the random marked rooted graph $\left(G^{\prime \prime}, \rho^{\prime}, m\right)$ is reversible. Thus, since $G^{\prime \prime}$ contains a vertex in $L$ a.s., the random walk on $G^{\prime \prime}$ must visit $L$ a.s. We conclude by noting that the random walk on $M^{\dagger}$ started at a vertex $f \in F_{\text {fin }}$ and stopped when it first hits $F_{\infty}$ can be coupled with the random walk on $G^{\prime}$ started at the same $f$ and stopped when it first hits $L$ so that the two hitting times agree.

To study the FUSF, bias the reversible law of $\left(G^{\prime}, \rho^{\prime}\right)$ by $1 / \operatorname{deg}\left(\rho^{\prime}\right)$ to get a unimodular graph. Wilson's algorithm rooted at $\{\infty\} \cup F_{\infty}$ can be seen as generating a spanning forest $\mathfrak{F}^{\dagger}$ of $G^{\prime}$, starting with $\mathfrak{F}_{0}^{\dagger}=L$ and adding loop erased random walks. Proposition 5.17 implies that $\mathfrak{F}^{\dagger}$ is distributed as the dual of the free uniform spanning forest of $M$. The argument above implies that every component of $\mathfrak{F}^{\dagger}$ contains a unique vertex of $L$. Consider the mass transport where each vertex of $G^{\prime}$ sends a unit mass to the vertex of $L$ in its component of $\mathfrak{F}^{\dagger}$. Then every vertex sends mass 1 , and hence no vertex receives infinite mass. Thus, every component of $\mathfrak{F}^{\dagger}$ is finite, and the result follows.

Proofs when $M^{\dagger}$ is not locally finite. If $M$ is simply connected then Theorems 5.13 and 5.16 follow immediately from Proposition 5.18. If $M$ is recurrent and not simply connected, then $M$ and $\mathfrak{F}$ must both have two ends, giving Theorem 5.16. (Theorem 5.13 makes no claim about multiply connected maps.)

\subsection{Percolation and minimal spanning forests}

While the uniform spanning forests are related to random walks, the minimal spanning forests are related to bernoulli bond percolation. We recall some of the connections, and refer the reader to $[60, \S 11]$ for a detailed account. Bernoulli- $p$ bond percolation on $G$, denoted $\omega_{p}$, is the random subgraph of $G$ defined by keeping each edge of $G$ independently with probability $p$ and deleting the rest. The Bernoulli bond percolations $\left\{\omega_{p}\right\}_{p \in[0,1]}$ on a graph $G$ may be coupled monotonically by letting $\{U(e)\}_{e \in E}$ be a collection of i.i.d. Uniform([0,1]) random variables indexed by the edge set of $G$ and setting $\omega_{p}(e)=\mathbb{1}(U(e) \leq p)$ for every $e \in E$ and $p \in[0,1]$. The critical probability of $G$ is defined by

$$
p_{c}(G):=\inf \left\{p: \mathbb{P}\left(\omega_{p} \text { has an infinite connected component }\right)=1\right\} .
$$

It is well-known $[2,63]$ that if $(G, \rho)$ is an ergodic unimodular random rooted graph, then for each $p \in[0,1]$ the number of infinite connected components of $\omega_{p}$ for any is non-random and is in $\{0,1, \infty\}$ almost surely. Moreover, if $(G, \rho)$ is a unimodular random rooted graph and $p$ is such that $\omega_{p}$ has a unique infinite cluster almost surely, then $\omega_{p^{\prime}}$ has a unique 
infinite connected component almost surely for every $p^{\prime} \geq p[41,62,2]$. In light of this, the uniqueness threshold of a graph $G$ is defined to be

$$
p_{u}(G)=\inf \left\{p: \mathbb{P}\left(\omega_{p} \text { has a unique infinite connected component }\right)=1\right\} .
$$

Note that if $(G, \rho)$ is an ergodic, infinite unimodular random rooted graph, then the quantities $p_{c}(G)$ and $p_{u}(G)$ are non-random. It is of interest to determine which graphs have a nonuniqueness phase for Bernoulli bond percolation. The following was proven by Aldous and Lyons [2], following work by in the transitive setting by Aizenman, Kesten, and Newman [1], Burton and Keane [25], and Gandolfi, Keane, and Newman [34].

Theorem 5.19 (Theorem 1, (2) implies (16)). Let $(G, \rho)$ be an invariantly amenable unimodular random rooted graph. Then $\omega_{p}$ has at most one infinite cluster almost surely for every $p \in[0,1]$, and in particular $p_{c}=p_{u}$ almost surely.

Proof. This follows by combining Corollary 6.11, Lemma 6.12, Proposition 8.8, and Corollary 8.10 of $[2]$.

A long standing conjecture of Benjamini and Schramm [21] asserts conversely that every nonamenable transitive graph has $p_{c}<p_{u}$.

Lyons, Peres and Schramm [61] related the non-uniqueness phase to minimal spanning forests. Given a finite graph $G$ and an injective weight function $U: E(G) \rightarrow \mathbb{R}$ the minimal spanning tree of $G$ with respect to $U$ is defined to be the spanning tree $T$ of $G$ minimising the total weight $\sum_{e \in T} U(e)$. Equivalently, an edge $e$ of $G$ is contained in $T$ if and only if there does not exist a simple cycle in $G$ containing $e$ such that $e$ maximises $U(e)$ among the edges in this cycle. We write $\mathrm{MST}_{G}$ for the distribution on spanning trees of $G$ obtained by letting $T$ be the minimal spanning tree of $G$ with respect to weights $\{U(e)\}_{e \in E}$ given by i.i.d. Uniform $([0,1])$ random variables.

This extends to infinite graphs using exhaustions, as for uniform spanning trees. Given an exhaustion $\left(V_{n}\right)_{n \geq 0}$ of an infinite graph $G$, we define the free and wired minimal spanning forests as the weak limits

$$
\operatorname{FMSF}_{G}(S \subset T):=\lim _{n \rightarrow \infty} \operatorname{MST}_{G_{n}}(S \subset T)
$$

and

$$
\mathrm{WMSF}_{G}(S \subset T):=\lim _{n \rightarrow \infty} \operatorname{MST}_{G_{n}^{*}}(S \subset T) .
$$

The limits exist and do not depend on the choice of exhaustion. If $(G, \rho)$ is a unimodular random rooted graph and $\mathfrak{F}$ is a sample of either $\mathrm{WMSF}_{G}$ or $\mathrm{FMSF}_{G}$, then $\mathfrak{F}$ is a percolation on $(G, \rho)$. Unlike in the uniform case, both of the minimal spanning forests may also be defined directly on the infinite graph $G$ as follows. Let $\{U(e): e \in E\}$ be a collection of i.i.d. Uniform $([0,1])$ random variables indexed by the edge set of $G$. An edge $e$ of $G$ is included in free minimal spanning forest of $G$ if and only if it is not the heaviest edge in any simple cycle in $G$. An edge $e$ of $G$ is included in the wired minimal spanning forest of $G$ if and only if it is not the heaviest edge in any simple cycle in $G$ or in any bi-infinite simple path (or 'cycle through infinity') in $G$.

Theorem 5.20 (Theorem 1, equivalence of $(15)$ and $(16)$ ). Let $(G, \rho)$ be an infinite unimodular random rooted graph with $\mathbb{E}[\operatorname{deg}(\rho)]<\infty$. Then $p_{c}(G)<p_{u}(G)$ if and only if $\mathrm{FMSF}_{G} \neq \mathrm{WMSF}_{G}$ if and only if there is at most one infinite cluster in Bernoulli p-percolation on $G$, at every $p \in[0,1]$. 
Proof. Lyons, Peres and Schramm [61] proved that an infinite connected graph $G$ has $\mathrm{FMSF}_{G}=\mathrm{WMSF}_{G}$ if and only if for $\omega_{p}$ has a unique infinite cluster for Lebesgue-a.e. $p \in[0,1]$. Combining this with monotonicity of uniqueness [41, 2, Theorem 6.7] implies that if $\mathrm{FMSF}_{G}=\mathrm{WMSF}_{G}$ then $p_{c}(G)=p_{u}(G)$ and hence there is at most one infinite cluster at every $p \in[0,1]$ except possibly at $p=p_{c}$. If $G$ is invariantly amenable, there is at most one infinite cluster at every $p$ by Theorem 5.19 and so also at $p=p_{c}$. If $G$ is nonamenable, then by [2] there is no infinite cluster at $p=p_{c}$.

The minimal spanning forests share several properties with their uniform cousins:

Free dominates wired: The measure $\mathrm{FMSF}_{G}$ stochastically dominates the measure $\mathrm{WMSF}_{G}$ for every graph $G$.

Domination and subgraphs: let $H$ be a connected subgraph of $G$. Then the FMSF of $H$ stochastically dominates the restriction of the FMSF of $G$ to $H$.

Expected degree of the WMSF: The expected degree in the WMSF of root of any unimodular random rooted graph is two [2, Proposition 7.3].

Amenability and boundary conditions: If $(G, \rho)$ is an invariantly amenable random rooted graph, then $\mathrm{FMSF}_{G}=\mathrm{WMSF}_{G}$ almost surely [2, Proposition 18.14].

Planar duality: If $M$ is a simply connected map with locally finite dual $M^{\dagger}$ and $\mathfrak{F}$ is a sample of $\mathrm{FMSF}_{M}$, then $\mathfrak{F}^{\dagger}$ has law $\mathrm{WMSF}_{M^{\dagger}}[60, \S 11.5]$.

From the above, we deduce that if $(G, \rho)$ a unimodular random rooted graph, the measures $\mathrm{FMSF}_{G}$ and $\mathrm{WMSF}_{G}$ coincide almost surely if and only if the expected degree of $\rho$ in the FMSF of $G$ is two. As for the uniform spanning forests, we relate the expected degree of the FMSF to the average curvature. The proof of Theorem 5.21 is general enough to provide an alternative proof of Theorem 5.11 that does not rely on connectivity of the forest.

Theorem 5.21 (Theorem 1, equivalence of (1) and (15)). Let $(M, \rho)$ be an infinite simply connected unimodular random rooted map with $\mathbb{E}[\operatorname{deg}(\rho)]<\infty$ and let $\mathfrak{F}$ be a sample of $\mathrm{FMSF}_{M}$. Then

$$
\mathbb{E}\left[\operatorname{deg}_{\mathfrak{F}}(\rho)\right]=2-\frac{1}{\pi} \mathbb{K}(M, \rho) .
$$

In particular, $\mathrm{FMSF}_{M}=\mathrm{WMSF}_{M}$ almost surely if and only if $\mathbb{K}(M, \rho)=0$.

The equivalence of (16) and (2) in Theorem 1 can also be proven directly as follows. Let $(M, \rho)$ be a simply connected, invariantly nonamenable unimodular random rooted map. If $(M, \rho)$ has locally finite dual, we deduce that $p_{c}(M)<p_{u}(M)$ by applying the following two results.

Theorem 5.22 (Benjamini, Lyons, Peres and Schramm [12, 11]; Aldous and Lyons [2]). Let $(G, \rho)$ be an invariantly nonamenable unimodular random rooted graph with $\mathbb{E}[\operatorname{deg}(\rho)]<\infty$. Then $\omega_{p_{c}}$ does not contain any infinite connected components almost surely.

Theorem 5.23 (Benjamini and Schramm [19, Theorem 3.1]). Let $(M, \rho)$ be an invariantly nonamenable, simply connected unimodular random rooted map with locally finite dual $M^{\dagger}$ and suppose that $\mathbb{E}[\operatorname{deg}(\rho)]<\infty$. Then $\omega_{p}$ has a unique infinite connected component if and only if every component of $\omega_{p}^{\dagger}$ is finite. It follows that

$$
p_{u}(M)=1-p_{c}\left(M^{\dagger}\right)
$$

almost surely and that $\omega_{p_{u}}$ contains a unique infinite connected component almost surely. 
Since at $p_{c}$ there is no infinite cluster, and at $p_{u}$ one exists, it follows that $p_{c}<p_{u}$. We remark that Benjamini and Schramm proved their theorem for transitive planar graphs, but their proof extends immediately to our setting. Since we provide an alternative proof via the curvature, we omit further details.

If $M$ does not have locally finite dual, then it must have infinitely many infinite faces by Lemma 3.13, so that the underlying graph of $M$ is infinitely ended. In this case, we have that $p_{u}(M)=1$ almost surely (see Proposition 7.5), while $p_{c}(M)<1$ by Theorem 5.22.

Thus, we have the following.

Corollary 5.24 (Theorem 1, (2) implies (16)). Let $(M, \rho)$ be an infinite, simply connected, invariantly nonamenable unimodular random rooted map with $\mathbb{E}[\operatorname{deg}(\rho)]<\infty$. Then $p_{c}(M)<$ $p_{u}(M)$ almost surely.

\subsection{Expected degree formula}

We prove Theorem 5.21. Every property of the minimal spanning forests that we use also holds for the uniform spanning forests, so that we also obtain an alternative proof of Theorem 5.11 that does not rely on Theorem 5.13.

Proof of Theorem 5.21 (and Theorem 5.11).

Locally finite dual case. Let $\omega$ be a percolation on $(M, \rho)$, and let $\omega^{\dagger}=\left\{e^{\dagger} \in E^{\dagger}: e \notin \omega\right\}$ be the dual percolation. As in Section 2.5, let $\eta$ be chosen uniformly at random from the set $E_{\rho}^{\rightarrow}$ of oriented edges of $M$ emanating from $\rho$, let $\rho^{\dagger}=\eta^{r}$, let $\mathbb{P}^{\text {rev }}$ be the $\operatorname{deg}(\rho)$-biasing of $\mathbb{P}$ and let $\mathbb{P}^{\dagger}$ be the $\operatorname{deg}\left(\rho^{\dagger}\right)^{-1}$ biasing of $\mathbb{P}^{r e v}$, so that $\left(M^{\dagger}, \rho^{\dagger}\right)$ is a unimodular random rooted map under $\mathbb{P}^{\text {rev }}$. We write $\mathbb{E}^{\dagger}$ for the expectation operator associated to $\mathbb{P}^{\dagger}$.

Lemma 5.25. For any percolation $\omega$ we have

$$
\mathbb{E}\left[\operatorname{deg}_{\omega}(\rho)\right]=\mathbb{E}[\operatorname{deg}(\rho)]-\mathbb{E}\left[\sum_{f \perp \rho} \operatorname{deg}(f)^{-1}\right] \mathbb{E}^{\dagger}\left[\operatorname{deg}_{\omega^{\dagger}}\left(\rho^{\dagger}\right)\right] .
$$

Proof. Observe that, since $\eta$ is uniformly distributed on $E_{\rho}^{\rightarrow}$ conditional on $(M, \rho)$, we have

$$
\mathbb{E}\left[\operatorname{deg}_{\omega}(\rho)\right]=\mathbb{E}[\operatorname{deg}(\rho) \mathbb{1}(\eta \in \omega)]=\mathbb{E}\left[\operatorname{deg}(\rho)\left(1-\mathbb{1}\left(\eta^{\dagger} \in \omega^{\dagger}\right)\right)\right],
$$

and so

$$
\mathbb{E}\left[\operatorname{deg}_{\omega}(\rho)\right]=\mathbb{E}[\operatorname{deg}(\rho)]\left(1-\mathbb{P}^{\text {rev }}\left(\eta^{\dagger} \in \omega^{\dagger}\right)\right) .
$$

Similarly, since under the measure $\mathbb{P}^{\dagger}$ and conditional on $\left(M^{\dagger}, \rho^{\dagger}\right), \eta^{\dagger}$ is uniformly distributed on $E_{\rho^{\dagger}}^{\rightarrow}$,

$$
\mathbb{E}^{\dagger}\left[\operatorname{deg}_{\omega^{\dagger}}\left(\rho^{\dagger}\right)\right]=\mathbb{E}^{\dagger}\left[\operatorname{deg}\left(\rho^{\dagger}\right)\right] \mathbb{P}^{\text {rev }}\left(\eta^{\dagger} \in \omega^{\dagger}\right)
$$

It follows that

$$
\mathbb{E}\left[\operatorname{deg}_{\omega}(\rho)\right]=\mathbb{E}[\operatorname{deg}(\rho)]\left(1-\frac{\mathbb{E}^{\dagger}\left[\operatorname{deg}\left(\rho^{\dagger}\right)\right]}{\mathbb{E}[\operatorname{deg}(\rho)]} \mathbb{E}^{\dagger}\left[\operatorname{deg}_{\omega^{\dagger}}\left(\rho^{\dagger}\right)\right]\right) .
$$

Applying the expected degree formula (2.3), we deduce that

$$
\mathbb{E}\left[\operatorname{deg}_{\omega}(\rho)\right]=\mathbb{E}[\operatorname{deg}(\rho)]\left(1-\frac{\mathbb{E}\left[\sum_{f \perp \rho} \operatorname{deg}(f)^{-1}\right]}{\mathbb{E}[\operatorname{deg}(\rho)]} \mathbb{E}^{\dagger}\left[\operatorname{deg}_{\omega^{\dagger}}\left(\rho^{\dagger}\right)\right]\right),
$$

which rearranges to give the desired expression. 
Let $\mathfrak{F}$ have law $\mathrm{FMSF}_{M}$. By Proposition 5.9, the dual forest $\mathfrak{F}^{\dagger}$ is distributed according to $\mathrm{WMSF}_{M^{\dagger}}$. Since the expected degree at the root of the WMSF in any unimodular random rooted graph is 2 , we have $\mathbb{E}^{\dagger}\left[\operatorname{deg}_{\mathfrak{F}^{\dagger}}\left(\rho^{\dagger}\right)\right]=2$ and consequently, by Lemma 5.25,

$$
\mathbb{E}\left[\operatorname{deg}_{\mathfrak{F}}(\rho)\right]=\mathbb{E}[\operatorname{deg}(\rho)]-2 \mathbb{E}\left[\sum_{f \perp \rho} \operatorname{deg}(f)^{-1}\right]=\mathbb{E}\left[\sum_{f \perp \rho} \frac{\operatorname{deg}(f)-2}{\operatorname{deg}(f)}\right]=2-\frac{1}{\pi} \mathbb{K}(M, \rho) .
$$

This completes the proof in the case that the dual of $M$ is locally finite.

Non-locally finite dual case. We begin with an upper bound that holds in greater generality. Given a unimodular random rooted map $(M, \rho)$ with $M^{\dagger}$ not locally finite, recall the construction of the map $M^{\prime}$ from the proof of Proposition 4.5, in which each infinite face of $M$ is triangulated.

Lemma 5.26. If $\omega$ is a percolation on an infinite unimodular random rooted map $(M, \rho)$ that is almost surely a spanning forest of $M$, then

$$
\mathbb{E}\left[\operatorname{deg}_{\omega}(\rho)\right] \leq 2-\frac{1}{\pi} \mathbb{K}(M, \rho) .
$$

Proof. If $M$ has a locally finite dual, then every component of $\omega^{\dagger}$ is infinite. It follows from $\left[2\right.$, Theorem 6.1] that $\mathbb{E}^{\dagger}\left[\operatorname{deg}_{\omega^{\dagger}}\left(\rho^{\dagger}\right)\right] \geq 2$, and the claim follows from Lemma 5.25. If $M^{\dagger}$ is not locally finite, note that $\omega$ is also a percolation on the map $M^{\prime}$, which has the same curvature as $M$, and the claim follows as above.

For the reverse inequality we approximate $M$ by maps with locally finite duals. Delete each edge of $M^{\prime}$ not in $E$ independently with probability $1-\varepsilon$, and call the resulting map $M_{\varepsilon}^{\prime}$. Clearly $\left(M_{\varepsilon}^{\prime}, \rho\right)$ converges locally to $(M, \rho)$ as $\varepsilon \rightarrow 0$, and it is not hard to see that $M_{\varepsilon}^{\prime}$ has locally finite dual a.s. for every $\varepsilon>0$. Let $\mathfrak{F}_{\varepsilon}$ be a sample of $\operatorname{FMSF}_{M_{\varepsilon}^{\prime}}$. Since $\left(M_{\varepsilon}^{\prime}, \rho\right)$ has locally finite dual, we have that

$$
\mathbb{E}\left[\operatorname{deg}_{\mathfrak{F}_{\varepsilon}}(\rho)\right]=2-\frac{1}{\pi} \mathbb{K}\left(M_{\varepsilon}^{\prime}, \rho\right)=2-\frac{1}{\pi} \mathbb{K}(M, \rho),
$$

where the second equality follows from Proposition 4.5.

Since the underlying graph of $M$ is a subgraph of the underlying graph of $M_{\varepsilon}^{\prime}$, the forest $\mathfrak{F}$ stochastically dominates the restriction $\mathfrak{F}_{\varepsilon} \cap E$ for every $\varepsilon>0$. Hence, by the dominated convergence theorem, we have

$$
\begin{aligned}
\mathbb{E}\left[\operatorname{deg}_{\mathfrak{F}}(\rho)\right] & \geq \mathbb{E}\left[\operatorname{deg}_{\mathfrak{F}_{\varepsilon}}(\rho)\right]-\mathbb{E}\left[\operatorname{deg}_{M_{\varepsilon}^{\prime}}(\rho)-\operatorname{deg}_{M}(\rho)\right] \\
& =2-\frac{1}{\pi} \mathbb{K}(M, \rho)-\mathbb{E}\left[\operatorname{deg}_{M_{\varepsilon}^{\prime}}(\rho)-\operatorname{deg}_{M}(\rho)\right] \underset{\varepsilon \rightarrow 0}{\longrightarrow} 2-\frac{1}{\pi} \mathbb{K}(M, \rho),
\end{aligned}
$$

completing the proof.

\section{The Conformal Type}

Given a map $M$ such that every face of $M$ has degree at least three, we may form a surface $S(M)$ (as described in Section 2.1) by gluing regular unit polygons together according to the combinatorics of $M$, the boundaries of these polygons becoming the edges of $M$ embedded in $S(M)$. Recall that we consider the upper half-space $\{x+i y \in \mathbb{C}: y>0\}$ with edges $\{[n, n+1]: n \in \mathbb{Z}\}$ to be a regular $\infty$-gon. The surface $S(M)$ is endowed naturally with a conformal structure by defining an atlas as follows. 
- For each face $f$ of $M$, we take as a chart the identity map from the interior of the regular polygon corresponding to $f$ to itself.

- For each edge $e$ of $M$, we define an open neighbourhood of the interior of $e$ in $S$ by adding to $e$ the two triangles formed by the endpoints of $e$ and the centres of the two faces adjacent to $e$ (if either face is infinite, we interpret this triangle to be the infinite strip starting at $e$ and perpendicular to the boundary of the face). To define a coordinate chart on this neighbourhood, we simply place the two triangles next to each other in the plane. The reason to take only a triangle and not the entire face is that this chart is well defined even if both sides of $e$ are incident to the same face.

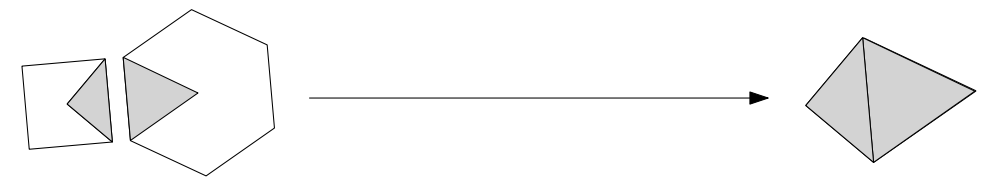

- For each vertex $v$ of $M$, we define an open neighbourhood of $v$ in $S$ similarly by intersecting the corners of the faces adjacent to $v$ with open discs of radius $1 / 2$ centred at $v$. We define a chart on this neighbourhood by first laying the corners out sequentially around the origin (with possible overlapping), and then applying the function $z \mapsto z^{2 \pi / \theta(v)}$, suitably interpreted to get an injective map into the plane. The radius is chosen so that this definition remains valid when multiple corners of the same face are located at the same vertex.
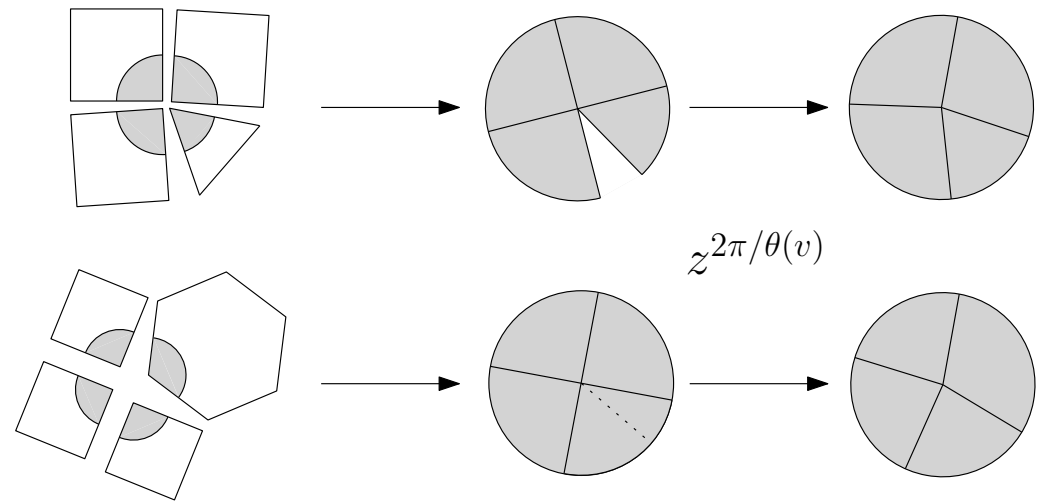

The coordinate changes are easily seen to be analytic, so that this atlas does indeed define a Riemann surface structure on $S(M)$. We denote this Riemann surface by $\mathcal{R}(M)$.

The definition can be extended to maps containing faces of degree 1 and 2 in various ways. One of these is as follows: Given a map $M$, let $\hat{M}$ be obtained from $M$ by triangulating faces of degree 1 or 2 by adding a vertex inside each face of $M$ that has degree 1 or 2 , and connecting this vertex to each corner of the face. Every face of $\hat{M}$ has degree at least three, and we define $\mathcal{R}(M)=\mathcal{R}(\hat{M})$. The map $M$ can be embedded in $\mathcal{R}(M)$ by restricting the natural embedding of $\hat{M}$ into $\mathcal{R}(\hat{M})$. An alternative way to deal with general maps is to apply this triangulation procedure to all faces of finite degree, resulting in a map with only faces of degree 3 or $\infty$.

We remark that there are many other natural (and inequivalent) ways to associate Riemann surfaces to maps. For example, we could associate to each face of $M$ a disc of circumference $k$, with boundary split into $k$ arcs of length one corresponding to the edges, and glue adjacent faces according to arc length along their shared edges. For concreteness we proceed with the Riemann surface defined above, though our arguments apply with minor adaptations for many other surfaces associated with $M$. 
If $M$ is simply connected, the uniformization theorem implies that $\mathcal{R}(M)$ is conformally equivalent to the sphere, the plane or the disc, and we call $M$ conformally elliptic, parabolic, or hyperbolic accordingly. The resulting embedding of $M$ into the sphere, plane, or disc given by uniformizing $\mathcal{R}(M)$ is unique up to Möbius transformations of the sphere, plane or disc as appropriate. This is referred to as the conformal embedding of $M$. Conformal embeddings of unimodular random planar maps are conjectured to play a key role in the theory of two-dimensional quantum gravity, see for example [27] and references therein. Elliptic maps are necessarily finite - the focus of this section is to distinguish conformally parabolic maps from conformally hyperbolic maps.

The conformal parabolicity of an infinite planar map is equivalent to the recurrence of Brownian motion on $\mathcal{R}(M)$, which can heuristically serve as a proxy for recurrence of the map itself. Gill and Rohde [37] proved that every Benjamini-Schramm limit of finite planar maps with uniformly bounded face degrees is conformally parabolic almost surely. The main result of this section generalises and, together with Corollary 3.5, provides a converse to their result.

Theorem 6.1 (Theorem 1, equivalence of (9) and (2) in the simply connected case.). Let $(M, \rho)$ be an infinite, ergodic, simply connected unimodular random rooted map with $\mathbb{E}[\operatorname{deg}(\rho)]<\infty$. Then $M$ is conformally parabolic almost surely if and only if $(M, \rho)$ is hyperfinite.

See Section 7 for the equivalence of (9) and (2) for multiply connected maps.

Remark. Although it is natural to try to prove Theorem 6.1 as an application of Theorem 4.10, it is difficult to verify the required integrability condition on the geodesic curvatures of edges. Indeed, we were able to do so only under a stronger moment assumption on $\operatorname{deg}(\rho)$.

Proof of Theorem 6.1, Conformal parabolicity implies hyperfiniteness. Let $(M, \rho)$ be a unimodular random rooted map such that $M$ is a.s. conformally parabolic. We claim that $(M, \rho)$ is hyperfinite (finite expected degree is not needed for this implication). Let $z$ be a conformal embedding of $M$ into the plane, which is uniquely determined up to translation and scaling.

Consider the image of the vertices $z(V)$. We first claim that the convex hull $C$ of $z(V)$ is either the full plane, a half plane, or a doubly infinite strip; the remainder of the proof will be split into cases accordingly. Indeed, since $z(V)$ is locally finite, if $C$ is any other set then there must exist a vertex $v$ of $M$ such that $z(v)$ is an extreme point of $C$. The external angles of $C$ at these vertices are positive, add up to at most $2 \pi$, and are independent of the choice of $z$. Transporting a mass $\alpha$ from every vertex of $M$ to every vertex such that $z(v)$ is an extreme point of $C$ with external angle $\alpha$ gives a contradiction since the mass out from each vertex is bounded while vertices such that $z(v)$ is an extreme point of $C$ receive infinite mass. We now show that in each of the three cases, $M$ is hyperfinite.

Case 1: $C$ is a strip. Without loss of generality we may assume the strip is parallel to $\mathbb{R}$. (Otherwise apply the subsequent argument to a suitable rotation of $z$.) Consider the bi-infinite path on $z(V)$ visiting the vertices of $M$ in the order given by their real part (breaking ties randomly). This defines a unimodular coupling between $(M, \rho)$ and $\mathbb{Z}$, and it follows from Proposition 3.12 that $(M, \rho)$ is hyperfinite in this case.

Case 2: $C$ is a half-plane. Without loss of generality we may assume that $C$ is the upper half-plane. We will use the linear structure of the boundary of $H$ to show that $(M, \rho)$ is coupling equivalent to $\mathbb{Z}$, so that it will follow from Proposition 3.12 that $(M, \rho)$ is hyperfinite. 
Since $z(V)$ is locally finite, there exist vertices $v$ such that the cone $\{w: \arg (z(v)-w) \in$ $(\pi / 3,2 \pi / 3)\}$ contains no other points of $z(V)$. (The choice of angles is arbitrary; any cone with finite intersection with the half-plane will do.) Call such vertices exposed.

It follows from ergodicity and unimodularity that a.s. there are infinitely many exposed vertices. Observe that, again using the fact that $z(V)$ is locally finite, for every bounded interval $[a, b]$, there exist at most finitely many exposed vertices $v$ that have $\Re z(v) \in[a, b]$. Define a path whose vertices are the exposed vertices of $M$, arranged in order of their real part. It follows that $(M, \rho)$ and $\mathbb{Z}$ are coupling equivalent as claimed.

Case 3: $C$ is the full plane. We define $D$ to be the Delaunay tessellation with vertex set given by the set of points $z(V)$. That is, $D$ is a map embedded in $\mathbb{C}$ with vertex set $z(V)$. Faces of $D$ correspond to discs (or half planes) with no vertices in their interior and at least 3 vertices on their boundary, so that each face is circumscribed in the corresponding disc. Note that the isomorphism class of $(D, \rho)$ is independent of the choice of the conformal embedding, which is unique up to homothety, and so $(M, \rho)$ and $(D, \rho)$ are coupling equivalent.

Since every half plane intersects $z(V)$, the faces of $D$ are all finite. If $D$ itself is not locally finite, then for every infinite degree vertex $v$ of $D$ there exists an infinite sequence of vertices $u_{i}$ and closed discs or half-planes $C_{i}$ such that $C_{i}$ contains both $z(v)$ and $z\left(u_{i}\right)$ in its boundary and no points of $z(V)$ in its interior. By taking a subsequential limit, it follows from the fact that $z(V)$ is locally finite that there must exist a half-space containing $z(v)$ in its boundary and no points of $z(V)$ in its interior, contradicting the assumption that $C$ is the full plane.

Thus $D$ and its dual are locally finite. In this case, it follows from the measurability of the conformal embedding that $(D, \rho)$ is a unimodular random rooted map, and that $(M, \rho)$ and $(D, \rho)$ are coupling equivalent. Thus, by Proposition 3.12, it suffices to prove that $(D, \rho)$ is hyperfinite.

Define a mass transport as follows. For each vertex $u$ and face $f$ of $D$ incident to $u$, let $\operatorname{ang}(f, u)$ be the angle of the corner of $f$ at $u$. Transport a mass of $\operatorname{ang}(f, u) / \operatorname{deg}(f)$ from $u$ to each of the vertices incident to $f$, including $u$ itself. The mass sent out by each vertex $u$ is $2 \pi$. Since each face is a polygon in the plane, the mass received is

$$
\sum_{f \perp u} \frac{\text { sum of internal angles of } f}{\operatorname{deg}(f)}=\sum_{f \perp u} \frac{\operatorname{deg}(f)-2}{\operatorname{deg}(f)} \pi \text {. }
$$

Applying the mass-transport principle yields that the average curvature of $(D, \rho)$ is zero. It follows from the equivalence of items (1) and (2) of Theorem 1 that $(D, \rho)$ is hyperfinite as claimed.

For the converse, suppose $M$ is a conformally hyperbolic, so that we have a conformal map $\phi$ from $\mathcal{R}(M)$ to the hyperbolic plane $\mathbb{H}$, which is unique up to isometries. Our strategy is to use $\phi$ to give a unimodular coupling of $M$ with another map which is known to be invariantly nonamenable. One possibility is to use (as above) the Delaunay tessalation on $\phi(V)$. The mass transport above would be used to prove that $D$ has negative mean curvature, and hence is nonamenable. The difficulty lies in establishing the local finiteness of the resulting map and its dual. Indeed with no further assumptions the resulting map can have infinite faces, and a modification is needed.

A crucial first step is establishing that $\phi(V)$ is reasonably dense in $\mathbb{H}$, in the sense that there is a unimodular partition of $\mathbb{H}$ which assigns finite mean area to every vertex. For each corner $c$ at each vertex $v$ of $M$, let $U_{c}$ be the quadrilateral in the face of $c$ with corners given 

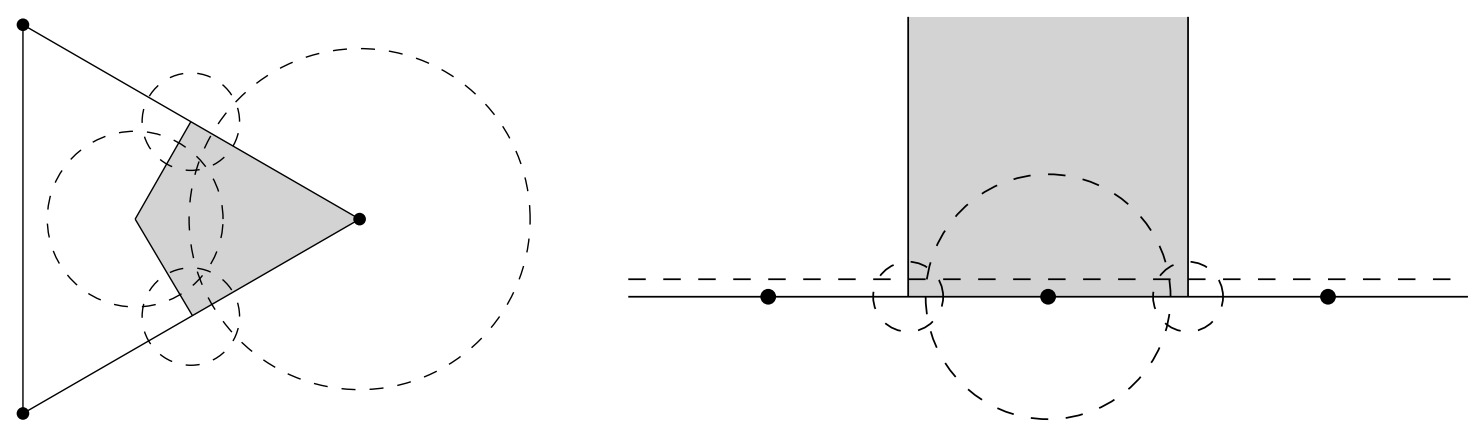

Figure 7. The covering of $U_{e}$ (grey) by discs (dashed boundaries) used in the proof of Lemma 6.2. Left: the case that $e^{r}$ has degree three. Right: the case that $e^{r}$ has infinite degree.

by $v$, the centre of the face, and the midpoints of the two edges forming the corner. If the face is infinite, we take $U_{c}$ to be the half-infinite strip in $f$ with right angled corners at the mid-points of the two edges of the corner (see Figure 7). We define a partition of $\mathcal{R}(M)$ by

$$
U_{v}=\bigcup_{c \perp v} U_{c}
$$

Given sets $K \subset S \subset \mathcal{R}(M)$, with $S$ simply connected, let $\phi_{S}$ be a conformal equivalence between $S$ and the unit disc. We write area $(K, S)$ for the hyperbolic area of $\phi_{S}(K)$ (which is independent of the choice of $\left.\phi_{S}\right)$. We also write area $\mathbb{H}(K)=\operatorname{area}_{\mathbb{H}}(K, \mathcal{R}(M))$. Recall the Schwarz-Pick Lemma: If $\mathcal{R}$ is a Riemann surface that is conformally equivalent to the disc, $S$ is a simply connected open subset of $\mathcal{R}$, and $K$ is a Borel subset of $S$, then the hyperbolic area of $K$ considered as a subset of $S$ is greater than or equal to the hyperbolic area of $K$ considered as a subset of $\mathcal{R}$. Thus $\operatorname{area}_{\mathbb{H}}(K) \leq \operatorname{area}_{\mathbb{H}}(K, S)$.

Lemma 6.2. There exists a constant $C$ such that the following holds. Let $M$ be a conformally hyperbolic, simply connected map such that every face of $M$ has degree at least three. Then for every vertex $v$,

$$
\operatorname{area}_{\mathbb{H}}\left(U_{v}\right) \leq C \operatorname{deg}(v) .
$$

We shall require the following fact: For every $0<\varepsilon<1$, the hyperbolic area of the set $\{z \in \mathbb{C}:|z|<1-\varepsilon\}$, considered as a subset of the open unit disc, is given by

$$
\frac{4 \pi(1-\varepsilon)^{2}}{1-(1-\varepsilon)^{2}} \leq \frac{2 \pi}{\varepsilon}
$$

Proof. For each corner $c$ incident to vertex $v$ and face $f$, let $U_{c}^{1}, \ldots, U_{c}^{4}$ be the following subsets of the polygon associated to $f$ :

(1) $U_{c}^{1}$ and $U_{c}^{2}$ are the $1 / 8$ neighbourhoods of the midpoints of the edges of $c$.

(2) $U_{c}^{3}$ is the $7 / 16$ neighbourhood of $v$.

(3) If $f$ has finite degree, then $U_{c}^{4}$ is the intersection of $U_{c}$ with the disc that is centred at the centre of the polygon corresponding to $f$ and that reaches distance $1 / 16$ from the boundary of the polygon. If $f$ has infinite degree, we let $U_{c}^{4}$ be the part of $U_{c}$ at distance at least $1 / 16$ from the boundary of the half-plane corresponding to $f$ ).

It is easily verified by elementary trigonometry that the $U_{c}$ is contained in the union $\bigcup_{i=1}^{4} U_{c}^{i}$. See Figure 7 for an illustration. 
We first prove that each of $U_{c}^{1}, U_{c}^{2}$, and $U_{c}^{4}$ have uniformly bounded hyperbolic areas. For $U_{c}^{1}$, let $S$ be the ball of radius $1 / 4$ around the midpoint of an edge $e$, so that $U_{c}^{1} \subset S$. Recall that for an edge $e$, the chart at $e$ includes the two triangles formed by the endpoints of $e$ and the centres of the faces adjacent to $e$. These triangles have disjoint interiors even if the same face is on both sides of $e$. It is easily verified that $S$ is always contained in the domain formed by placing the two triangles together. Since $U_{c}^{1}$ is the ball of radius $1 / 8$ around the midpoint of $e$, it follows by Schwarz-Pick and (6.1) that $\operatorname{area}_{\mathbb{H}}\left(U_{c}^{1}\right) \leq \operatorname{area}_{\mathbb{H}}\left(U_{c}^{1}, S\right) \leq C$ for some constant (namely, $4 \pi$ ). The corresponding claim for $U_{c}^{2}$ follows similarly.

We next claim that area $\left(U_{c}^{4}\right) \leq 16$. If the corresponding face $f$ has finite degree, let $S$ be the disc circumscribed in the polygon of $f$, and let $K$ be the disc that has the same centre as $S$ and radius 1/16 smaller than that of $S$. The circumference of $S$ is less than the perimeter of $f$, and hence the radius $\rho$ of $S$ is at most $\operatorname{deg}(f) / 2 \pi$. Thus, it follows from (6.1) that

$$
\operatorname{area}_{\mathbb{H}}(K, S) \leq 16 \operatorname{deg}(f) .
$$

Since $U_{c}^{4}$ is a wedge of $K$, it follows by the symmetry of $S$ that $\operatorname{area}_{\mathbb{H}}\left(U_{c}^{4}, S\right) \leq 16$. By Schwarz-Pick, the same bound holds for area $\mathbb{H}_{\mathbb{H}}\left(U_{c}^{4}\right)$. If $f$ has infinite degree, then as a subset of $f, U_{c}^{4}$ is isometric to the set $\{x+i y: x \in[0,1], y \geq 1 / 16\}$ in the half plane representation of $\mathbb{H}$. This set is easily verified to have hyperbolic area 16. (One could also deduce this from the finite degree case by taking a limit.)

Finally, we claim that for every vertex $v$, the hyperbolic area of $U_{v}^{3}:=\bigcup_{c \perp v} U_{c}^{3}$ is at most $C \operatorname{deg}(v)$, where $C$ is a universal constant. Observe that $U_{v}^{3}$ is simply the ball of radius $7 / 16$ around $v$ in $\mathcal{R}(M)$. Let $S$ be the ball around $v$ of radius $1 / 2$. The chart provided for $S$ maps $U_{v}^{3}$ to a ball of radius $(7 / 16)^{2 \pi / \theta(v)}$, while $S$ gets mapped to the ball of radius $(1 / 2)^{2 \pi / \theta(v)}$ with the same centre. It follows from (6.1) that

$$
\operatorname{area}_{\mathbb{H}}\left(U_{v}^{3}, S\right) \leq \frac{2 \pi}{1-(7 / 8)^{2 \pi / \theta(v)}} \leq C \theta(v) \leq \pi C \operatorname{deg}(v),
$$

where $C$ is a constant. (Verifying that such a constant exists is a simple calculus exercise.) The claim now follows from Schwarz-Pick.

The lemma follows by combining the estimates given for $U_{v}^{3}$ and for each of $U_{c}^{1}, U_{c}^{2}$, and $U_{c}^{4}$ for each corner at $v$.

We remark that a simple additional argument gives the slightly stronger statement that $\operatorname{area}_{\mathbb{H}}\left(U_{c}\right)$ is uniformly bounded for each corner.

Proof of Theorem 6.1, Conformal hyperbolicity implies invariant nonamenability. We may assume that every face of $M$ has degree at least three. Otherwise, we consider the map $\hat{M}$ used to define $\mathcal{R}(M)$, all the faces of which have degree at least 3, and which is coupling equivalent to $M$. Let $\phi$ be a conformal equivalence between $\mathcal{R}(M)$ and the hyperbolic plane.

Let $Z$ be an independent Poisson point process of intensity 1 on the hyperbolic plane, and let $D$ be the Delaunay triangulation associated to $Z$. Since $D$ is a Poisson-Delaunay triangulation of the hyperbolic plane, $D$ is invariantly nonamenable (see $[16,4]$ ). To prove nonamenability of $M$ we give a unimodular coupling of $M$ and $D$, using a larger graph $G$, and conclude by applying Proposition 3.12.

The graph $G$ has vertex set $V \cup Z$, and has as edges the edges of $M$, the edges of $D$, and an edge connecting each $v \in V$ to every point $z \in Z \cap \phi\left(U_{v}\right)$. We mark the edges of $G$ according to which of these three types they come from. Note that the law of $(G, \rho)$ does not depend on the choice of $\phi$. It follows from Lemma 6.2 that the expected number of points in 
$Z \cap \phi\left(U_{\rho}\right)$ is finite. It is easily verified, using the measurability of the conformal embedding, that if we sample $(G, \rho)$ biased by $1+\left|Z \cap \phi\left(U_{\rho}\right)\right|$ and then let $\hat{\rho}$ be uniform on the set $\{\rho\} \cup\left(Z \cap \phi\left(U_{\rho}\right)\right)$, then the resulting random rooted graph $(G, \hat{\rho})$ is unimodular. Similarly, if we sample $(M, \rho)$ and $Z$ biased by $\left|Z \cap \phi\left(U_{\rho}\right)\right|$, and let $\rho^{\prime}$ be uniform on the set $Z \cap \phi\left(U_{\rho}\right)$, then the resulting graph $\left(D, \rho^{\prime}\right)$ is unimodular. Thus, we have defined a unimodular coupling between $(M, \rho)$ and $\left(D, \rho^{\prime}\right)$.

\section{Multiply-connected maps}

\subsection{The topology of unimodular random rooted maps.}

In this section we study multiply-connected unimodular random rooted maps. We begin by classifying the possible topologies of the surface associated to a unimodular random rooted map $(M, \rho)$. Biringer and Raimbault [22] classified the possible topologies of unimodular random rooted complete, orientable, hyperbolic surfaces. Their methods readily generalise to our setting, yielding the following theorem. In fact, the proof is slightly less technical in our setting, and we provide a quick sketch below.

Theorem 7.1 (Topology of unimodular random rooted maps). Let $(M, \rho)$ be an infinite unimodular random rooted map. Then the surface associated to $M$ is almost surely homeomorphic to one of the following surfaces: the plane, the cylinder, the Cantor tree, the infinite prison window, Jacob's ladder, or the blossoming Cantor tree. The type is determined by how many ends the surface has and whether or not it is planar.

Here, the cantor tree is a 'tree made of tubes', and is homeomorphic to the complement of the Cantor set in the sphere, the infinite prison window is 'the lattice $\mathbb{Z}^{2}$ made of tubes', Jacob's ladder is 'an infinite ladder made of tubes', and the blossoming Cantor tree is a Cantor tree with a handle attached near each bifurcation. See Figure 8 for illustrations. Be warned that homeomorphism is an extremely weak notion here. For example, 'the lattices $\mathbb{Z}^{d}$ made of tubes' are all homeomorphic to each other for all $d \geq 2$. We leave it to the reader to verify that each of the surfaces listed in Theorem 7.1 can occur as the almost sure homeomorphism class of a unimodular random rooted map.

In [22], Biringer and Raimbault must also allow for the surfaces above to be punctured at a locally finite set of points, corresponding to isolated ends of the surface. This does not occur in our setting, as the surfaces corresponding to unimodular random rooted maps do not have isolated ends.

Sketch of proof. An end $\xi$ of an infinite graph $G$ may be defined as a function that assigns a connected component $\xi_{K}$ of $G \backslash K$ to each finite set of vertices $K$ of $G$, and satifies the consistency condition that $\xi_{K^{\prime}} \subseteq \xi_{K}$ whenever $K^{\prime} \supseteq K$. The space of ends of $G$, denoted $\partial \mathscr{E}(G)$ is the topological space with the set of ends of $G$ as its underlying set and with a basis of open sets given by sets of the form $A_{K, W}=\left\{\xi\right.$ an end of $\left.G: \xi_{K}=W\right\}$, where $K \subset V$ is finite and $W \subset V$ is a connected component of $G \backslash K$. Note that the basis sets are also closed, so that the space of ends is always zero-dimensional, that is, its topology is induced by a basis of sets that are both open and closed. The space of ends of a surface is defined similarly, replacing instances of the word 'finite' by 'compact' above, and is also zero-dimensional.

It is well-known that every unimodular random rooted graph either has one, two, or infinitely many ends, and, in the last case, the space of ends does not have any isolated points 


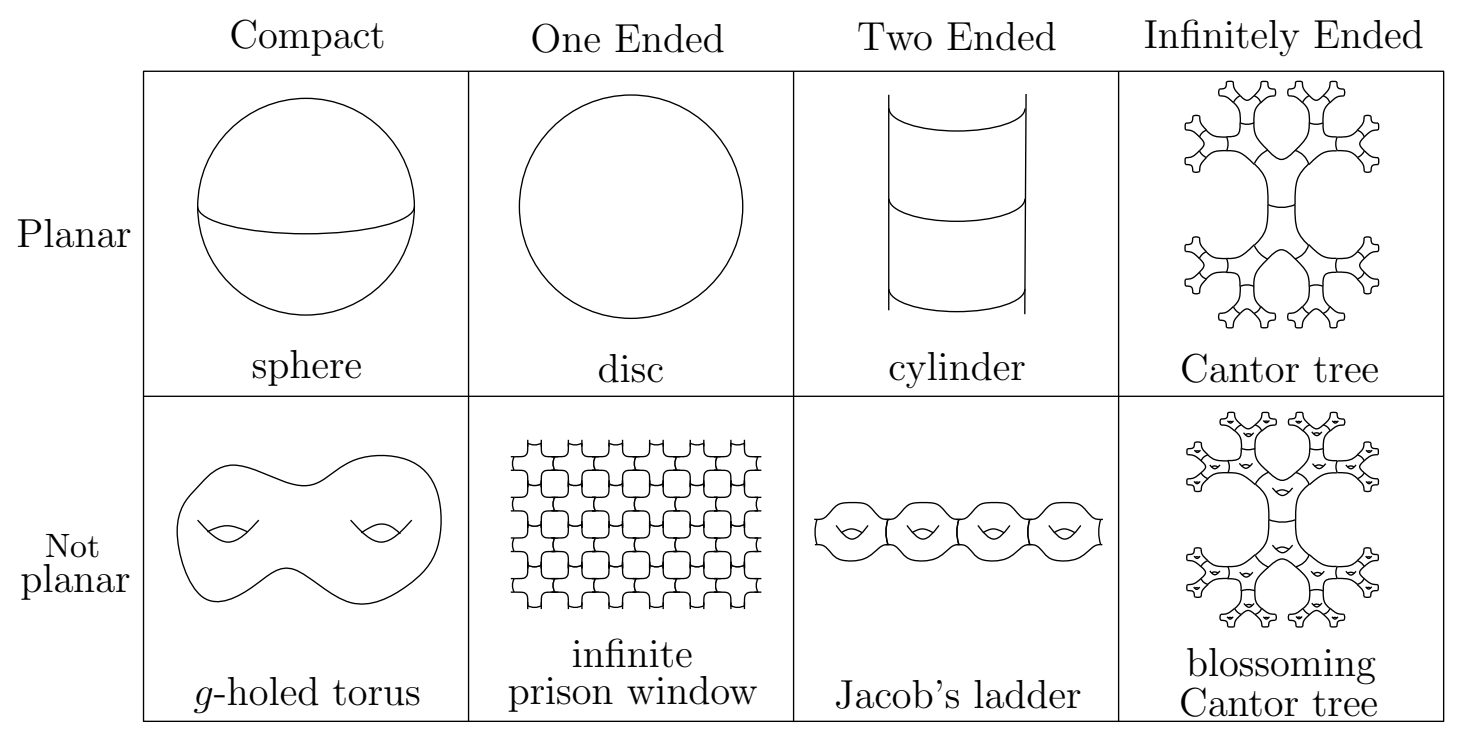

Figure 8. Possible topologies of a unimodular random map. The surface $S(M)$ associated to a unimodular random map $(M, \rho)$ is almost surely homeomorphic to one of the above.

[2, Proposition 6.10]. Since the space of ends of any graph is also compact, it follows in the last case that the space of ends is homeomorphic to the Cantor set (which, by Brouwer's Theorem [24], is the only compact, zero-dimensional Hausdorff space with no isolated points). A similar proof applies to show that if $(M, \rho)$ is a random rooted map with associated surface $S=S(M)$, then $S$ has either one, two, or infinitely many ends and in the last case the space of ends of $S$ is homeomorphic to a Cantor set. Next, a standard mass transport argument (each vertex sends unit mass to the 'nearest handle') shows that if $S$ contains handles, then the handles of $S$ accumulate towards every end of $S$. That is, if $S$ has handles then, for every compact subset $K$ of $S$, every non-precompact connected component of $S \backslash K$ contains a handle.

We next apply the classification theorem for non-compact surfaces due to Kerékjártó and Richards [65], which states that if two non-compact orientable surfaces $S_{1}$ and $S_{2}$ have the same number of handles (which in our case will be zero or infinity) and there exists a homeomorphism $\phi: \partial \mathscr{E}\left(S_{1}\right) \rightarrow \partial \mathscr{E}\left(S_{2}\right)$ such that the handles of $S_{1}$ accumulate to $\xi \in \partial \mathscr{E}\left(S_{1}\right)$ if and only if the handles of $S_{2}$ accumulate to $\phi(\xi)$, then $S_{1}$ and $S_{2}$ are homeomorphic and $\phi$ extends to a homeomorphism from the ends compactification of $S_{1}$ to the ends compactification of $S_{2}$. Thus, by the above discussion, the homeomorphism class of $S=S(M)$ is determined almost surely by its number of ends and by the existence or non-existence of handles. This yields the six different possibilities listed in the statement of the theorem (see Figure 6).

The main result of this section is that the average curvature of a unimodular random rooted map restricts the possible topologies of the map.

Theorem 7.2 (Topology and average curvature). Let $(M, \rho)$ be an ergodic unimodular random map. Then the almost sure conformal type of $M$ is determined by its average curvature: Either

(1) The average curvature of $(M, \rho)$ is positive, in which case $M$ is conformally elliptic and $S(M)$ is homeomorphic to the sphere almost surely, 
(2) the average curvature of $(M, \rho)$ is zero, in which case $M$ is conformally parabolic and or else $S(M)$ is homeomorphic to the plane, the cylinder, or the torus almost surely,

(3) the average curvature of $(M, \rho)$ is negative, in which case $M$ is conformally hyperbolic $S(M)$ is homoemorphic to either the plane, the blossoming Cantor tree, Jacob's ladder, the infinite prison window, or a compact surface of genus at least two almost surely.

The theorem will follow by combining Theorem 6.1, Theorem 7.1 and the notion of the universal cover of a map. Recall that a surjective, holomorphic function $\Pi: S \rightarrow S^{\prime}$ between two Riemann surfaces is a holomorphic covering if it is locally a homeomorphism, that is, if for every $x \in S$ there exists an open neighbourhood $U$ of $S$ such that the restriction of $\Pi$ to $U$ is a homeomorphism between $U$ and its image. Given a Riemann surface $S$, the universal cover of $S$ is a simply connected Riemann surface $\tilde{S}$ together with a covering $\Pi: \tilde{S} \rightarrow S$. The universal cover exists for any $S$, and is unique in the sense that if $\Pi^{\prime}: \tilde{S}^{\prime} \rightarrow S$ is another simply connected Riemann surface covering $S$, then there exists a conformal equivalence $\phi: \tilde{S}^{\prime} \rightarrow \tilde{S}$ such that $\Pi^{\prime}=\Pi \circ \phi$.

The universal cover of a map is defined analogously. Given a pair of maps $M=(G, \sigma)$ and $M^{\prime}=\left(G^{\prime}, \sigma^{\prime}\right)$, we say that a graph homomorphism $\phi: G \rightarrow G^{\prime}$ is a map homomorphism if $\sigma^{\prime} \circ \phi=\phi \circ \sigma$, and say that $\phi$ is a covering if for every vertex $v$ and every face $f$ of $M$, the restriction of $\phi$ to each of $\left\{e \in E^{\rightarrow}: e^{-}=v\right\}$ and $\left\{e \in E^{\rightarrow}: e^{r}=f\right\}$ is injective. The universal cover of a map $M$ is a simply connected map $\tilde{M}$ together with a covering $\pi: \tilde{M} \rightarrow M$. Every map has a universal cover, and the universal cover of a map $M$ is unique in the sense that if $\pi^{\prime}: \tilde{M}^{\prime} \rightarrow M$ is a covering from a simply connected map $\tilde{M}^{\prime}$ to $M$, there exists an isomorphism of maps $\phi: \tilde{M} \rightarrow \tilde{M}^{\prime}$ such that $\pi^{\prime} \circ \phi=\pi$.

We remark that the universal cover $\pi: \tilde{M} \rightarrow M$ of $M$ may be constructed by lifting every edge of $M$ in the surface $S(M)$ to the universal cover $\Pi: \tilde{S}(M) \rightarrow S(M)$ (see e.g. [42, p. 60] for the topological notion of path lifting). In particular, if $\mathcal{R}(\tilde{M})$ is the Riemann surface associated to $\tilde{M}$, then there exists a conformal equivalence $\Phi: \mathcal{R}(\tilde{M}) \rightarrow \tilde{\mathcal{R}}(M)$ such that $\Pi \circ \Phi \circ \tilde{z}=z \circ \pi$. (See e.g. [66, Section 9.2] for a direct construction.)

Covers of unimodular maps are unimodular. The following is proven in [4, Section 4.1].

Lemma 7.3. Let $(M, \rho)$ be a unimodular random rooted map. Let $(\tilde{M}, \pi)$ be the universal cover of $M$ and let $\tilde{\rho}$ be an arbitrary element of $\pi^{-1}(\rho)$. Then $(\tilde{M}, \tilde{\rho})$ is a unimodular random rooted map.

Proof of Theorem 7.2. Observe that $\kappa_{\tilde{M}}(\tilde{\rho})=\kappa(\rho)$ for any rooted map $(M, \rho)$, so that $\mathbb{K}(\tilde{M}, \tilde{\rho})=\mathbb{K}(M, \rho)$. By applying Theorem 6.1 and the classical theory of Riemann surfaces, we obtain that

- $\mathbb{K}(M, \rho)>0$ if and only if $M$ is finite and simply connected and $\tilde{\mathcal{R}}(M)=\mathcal{R}(M)$ is conformally equivalent to the sphere,

- $\mathbb{K}(M, \rho)=0$ if and only if $\tilde{\mathcal{R}}(M)$ is conformally equivalent to the plane, if and only if $\mathcal{R}(M)$ is conformally equivalent to one of the plane $\mathbb{C}$, the cylinder $\mathbb{C} / \mathbb{Z}$ or a torus $\mathbb{C} / \Lambda$ for some lattice $\Lambda \subset \mathbb{C}$, and

- $\mathbb{K}(M, \rho)<0$ if and only if $\tilde{\mathcal{R}}(M)$ is conformally equivalent to the hyperbolic plane (or disc). 
In the last case there are many possibilities for the conformal equivalence class $\mathcal{R}(M)$; any topology other than the sphere, plane, cylinder or a torus is possible. We conclude by applying the additional topological constraints on $\mathcal{R}(M)$ imposed by Theorem 7.1.

We next connect the topology of $S(M)$ to the number of ends of the underlying graph. If $S(M)$ is homeomorphic to the plane or the infinite prison window, it is possible for the underlying graph to have 1, 2, or infinitely many ends. (Examples in the plane case are $\mathbb{Z}^{2}, \mathbb{Z}$ and a regular tree; Add handles to these for examples with the topology of the infinite prison window.) The number of ends of the underlying graph of a map $M$ is at least the number of ends of $S(M)$. Thus if $S(M)$ is homeomorphic to the Cantor tree or the blossoming Cantor tree, and at least two ends if $S(M)$ is homeomorphic to the cylinder or Jacob's ladder. The case of two-ended $S(M)$ is covered by the following:

Lemma 7.4. Let $(M, \rho)$ be a unimodular random rooted map with $\mathbb{E}[\operatorname{deg}(\rho)]<\infty$. If $S(M)$ is homeomorphic to the cylinder or to Jacob's ladder almost surely, then the underlying graph of $M$ is recurrent and two-ended almost surely.

Proof. We bias by $\operatorname{deg}(\rho)$ and prove the equivalent statement for a reversible map. We may also assume that $(M, \rho)$ is ergodic. Suppose for contradiction that $M$ is either transient or has more than two ends almost surely. In the latter case, $M$ has infinitely many ends almost surely, is invariantly nonamenable, and hence transient almost surely.

Since $S(M)$ is two-ended almost surely, there exists some $r$ and $D$ such, with positive probability, the ball $B_{r}\left(M, X_{n}\right)$ of radius $r$ about $X_{n}$ in $M$ has degree sum at most $D$ and the complement $S(M) \backslash z\left(B_{r}\left(M, X_{n}\right)\right)$ has two non-precompact connected components, each of which is necessarily one-ended. Denote this event by $A_{n}$. By stationarity, $A_{n}$ occurs for infinitely many $n$ almost surely. Let $\left(n_{m}\right)_{m \geq 0}$ be a sequence of times at which $A_{n}$ occurs, and such that the balls $B_{r}\left(M, X_{n_{i}}\right)$ are disjoint. Let $W_{1}, W_{2}$ be the subgraphs of $M$ in the two unbounded components of $S \backslash z\left(B_{r}\left(M, X_{n_{0}}\right)\right)$. Since $M$ is transient almost surely, the simple random walk $\left(X_{n}\right)_{n \geq 0}$ eventually stays in one of the $W_{i}$, and so $W_{i}$ must be transient. However, the balls $B_{r}\left(M, X_{n_{m}}\right)$ yield an infinite collection of disjoint cutsets of degree sum at most $D$ separating $\rho$ from infinity in the subgraph induced by $W_{i}$. Thus, this graph is recurrent by the Nash-Williams criterion [60], a contradiction.

\subsection{Theorem 1 in the multiply-connected planar case}

In light of Theorem 7.1 we need to consider maps where $S(M)$ is homeomorphic to either the cylinder or the Cantor tree.

Suppose that $(M, \rho)$ is an infinite, multiply-connected unimodular random planar map with $\mathbb{E}[\operatorname{deg}(\rho)]<\infty$. If $\mathbb{K}(M, \rho)=0$, then the proof of Theorem 7.2 implies that $\mathcal{R}(M)$ is conformally equivalent to the cylinder. Lemma 7.4 then implies that the underlying graph of $M$ is recurrent and two-ended almost surely. We deduce that items 2, 4-6, 10, 11, 14 and 17 of Theorem 1 hold for $(M, \rho)$ as an immediate consequence of recurrence. The remaining items of Theorem 1 hold for $(M, \rho)$ as a consequence of invariant amenability.

Now suppose that $\mathbb{K}(M, \rho)<0$. In this case, Theorem 7.2 implies that $S(M)$ is almost surely homeomorphic to the Cantor tree and consequently that the underlying graph of $M$ is infinitely-ended almost surely by Lemma 3.8 , and hence that $(M, \rho)$ is invariantly nonamenable. The following two propositions, which are well-known to experts, show that the negations of items 11 and 16 hold for $(M, \rho)$. The negations of the remaining items of 
Theorem 1 follow from the negations of items 11 and 16 using implications that are valid for all unimodular random rooted graphs and that we have already reviewed earlier in the paper; see the green and blue arrows in Figure 1.

Proposition 7.5. Let $(G, \rho)$ be a unimodular random rooted graph with $\mathbb{E}[\operatorname{deg}(\rho)]<\infty$, and suppose that $G$ is infinitely ended almost surely. Then $p_{u}(G)=1$ almost surely.

Proof of Proposition 7.5. If $p_{c}=1$ the claim holds trivially, so suppose not. Fix some $p \in\left(p_{c}, 1\right)$, and let $I \subset V$ be the union of all infinite components of Bernoulli $p$-percolation. We first observe that for every finite $K \subset V$, each infinite component of $G \backslash K$ intersects $I$ almost surely. Indeed, if not, we get a contradiction by transporting unit mass from each vertex to the nearest vertex of $I$. There is some finite set $K$ so that $G \backslash K$ has multiple infinite connected components. With positive probability all edges incident to $K$ are closed, and thus there are multiple infinite percolation components.

Proposition 7.6. Let $(G, \rho)$ be a unimodular random rooted graph with $\mathbb{E}[\operatorname{deg}(\rho)]<\infty$, and suppose that $G$ is infinitely ended almost surely. Then $G$ admits non-constant harmonic functions of finite Dirichlet energy almost surely.

Proposition 7.6 will require the following lemma.

Lemma 7.7. Let $(G, \rho)$ be a unimodular random rooted graph, and suppose that $G$ is infinitely ended almost surely. Then for every finite set $K \subset V$, every infinite connected component $W$ of $G \backslash K$ is transient.

Proof. Since $G$ has infinitely many ends a.s., we have that $(G, \rho)$ is invariantly nonamenable and hence that $G$ is transient. Let $F(u, v)$ be the probability that a simple random walk started at $u$ hits $v$. Suppose for a contradiction that there exists a finite set $K \subset V$ and a recurrent infinite connected component $W$ of $G \backslash K$. It follows that for every vertex $v \in W$, a random walk started at $v$ must hit the set $K$ almost surely, and hence that, for each vertex $u$ of $K$, we have

$$
\inf _{v \in W} F(v, u) \geq \inf _{v \in W} F(v, K) \inf _{w \in K} F(w, u)>0 .
$$

Since $G$ is transient but $W$ is recurrent, we have that for every $\varepsilon>0$ there exist at most finitely many vertices $v \in W$ such that $F(u, v) \geq \varepsilon$, i.e. $F(u, v) \rightarrow 0$ as $v \rightarrow \infty$ in $W$. Symmetry of the Green's function $\operatorname{deg}(x) G(x, y)$ gives

$$
\frac{F(u, v) G(v, v)}{\operatorname{deg}(v)}=\frac{F(v, u) G(u, u)}{\operatorname{deg}(u)} .
$$

It follows that $G(v, v) / \operatorname{deg}(v)$ tends to infinity as $v \rightarrow \infty$ in $W$.

Choose $C$ sufficiently large that $G(\rho, \rho) / \operatorname{deg}(\rho) \leq C$ with positive probability. Define a mass transport by, for each vertex $v$ of $G$, transporting a mass of 1 to the closest vertex to $v$ that has $G(w, w,) / \operatorname{deg}(w) \leq C$. (If there are multiple choices of the vertex $w$, choose one uniformly.) Then every vertex sends a mass of at most one but, in the situation described, some vertices recieve an infinite amount of mass. This contradicts the mass transport principle.

Proof of Proposition 7.6. It is well-known that if $G$ is a graph and there exists a finite set $K$ such that $G \backslash K$ has multiple transient connected components, then $G$ admits a non-constant harmonic Dirichlet function: The probability that the walk eventually stays in a particular connected component is such a function (see [60, Exercise 9.23]). 


\section{Soficity}

We are now ready to prove Theorem 2. A unimodular random rooted graph $(G, \rho)$ is called strongly sofic if $(G, \rho, m)$ is sofic for every marking $m$ of $(G, \rho)$. (The constant marking shows this implies soficity.) One main ingredient to Theorem 2 is the following, which was proven for Cayley graphs of free groups by [23].

Theorem 8.1 (Bowen [23]; Elek [32]; Elek and Lippner [33]; Benjamini, Lyons and Schramm [15]). Every unimodular random rooted tree is strongly sofic.

The following theorem is an adaptation of a related theorem of Elek and Lippner [33] in the setting of group actions. Even in our setting, it is well-known to experts that treeable unimodular random graphs (i.e., unimodular random graphs admitting a unimodular random spanning tree) are sofic.

Theorem 8.2. Let $\left(G_{1}, \rho_{1}\right)$ and $\left(G_{2}, \rho_{2}\right)$ be coupling equivalent unimodular random rooted graphs. Then $\left(G_{1}, \rho_{1}\right)$ is strongly sofic if and only if $\left(G_{2}, \rho_{2}\right)$ is strongly sofic.

The proof can be summarised as follows: Suppose $\left(G_{1}, \rho_{1}\right)$ is strongly sofic, and let $m$ be a marking of $\left(G_{2}, \rho_{2}\right)$. We can encode both the structure of $G_{2}$ and the marks $m$ as a marking $\hat{m}$ of $\left(G_{1}, \rho_{1}\right)$. The strong soficity of $\left(G_{1}, \rho_{1}\right)$ allows us to approximate $\left(G_{1}, \rho_{1}, \hat{m}\right)$ by a sequence of finite graphs. We then use this sequence to define an approximating sequence for $\left(G_{2}, \rho_{2}, m\right)$. Making this argument rigorous takes some care.

Proof. Since $\left(G_{1}, \rho_{1}\right)$ and $\left(G_{2}, \rho_{2}\right)$ can both be considered as percolations on some unimodular random graph $(G, \rho)$, it suffices to prove that A unimodular graph is strongly sofic if and only if a connected percolation on it is strongly sofic. Explicitly, if $(G, \rho)$ is a unimodular random rooted graph and $\omega$ is an almost surely connected percolation on $G$, then $(G, \rho)$ is strongly sofic if and only if the unimodular random rooted graph $\left(H, \rho^{\prime}\right)$ obtained from $(\omega, \rho)$ by conditioning on $\omega(\rho)=1$ is strongly sofic.

First suppose that $(G, \rho)$ is strongly sofic and let $m$ be a marking of $\left(H, \rho^{\prime}\right)$. By Lemma 3.3, there exists a marking $m$ of $G$ such that $(G, \rho, \omega, m)$ is unimodular and such that the law of $\left(H, \rho^{\prime}, m\right)$ coincides with the law of $(\omega, \rho, m)$ conditional on $\omega(\rho)=1$. Since $(G, \rho)$ is strongly sofic, there exists a sequence of finite unimodular random marked graphs $\left(G_{n}, \rho_{n}, \omega_{n}, m_{n}\right)$ converging to $(G, \rho, \omega, m)$ in distribution. Let $\left(H_{n}, \rho_{n}^{\prime}, m_{n}\right)$ be the unimodular random rooted graph obtained from $\left(\omega_{n}, \rho_{n}, m_{n}\right)$ by conditioning on $\omega(\rho)=1$. The sequence $\left(H_{n}, \rho_{n}^{\prime}, m_{n}\right)$ converges to $\left(H, \rho^{\prime}, m\right)$ and, since $m$ was arbitrary, $\left(H, \rho^{\prime}\right)$ is strongly sofic.

Suppose conversely that $\left(H, \rho^{\prime}\right)$ is strongly sofic and let $m$ be an $\mathbb{X}$-marking of $(G, \rho)$. By Lemma 3.2, we may assume that $(G, \rho, \omega, m)$ is unimodular. For each vertex $u$ of $G$, let $v(u)$ be chosen uniformly from the set of vertices in $\omega$ that minimize the graph distance to $u$, and for each vertex $v$ of $\omega$ let $U_{v}=\{v(u)=v\}$. Transporting mass 1 from $u$ to $v(u)$ for every vertex $u$ of $G$ shows that $\mathbb{E}\left|U_{\rho}\right|<\infty$, and in particular $U_{v}$ is finite for every vertex $v$ of $\omega$ almost surley. Conditional on $(G, \rho, \omega, m)$, let $\{U(v): v \in V\} \cup\{U(e): e \in E\}$ be a collection i.i.d. uniform $[0,1]$ random variables indexed by the vertices and edges of $G$, and for each vertex $v$ of $G$ such that $\omega(v)=1$, define

$$
\hat{m}(v)=\left\{(U(u), m(u),\{(U(e), m(e)): e \text { is an edge incident to } u \text { in } G\}): u \in U_{v}\right\} .
$$

If we denote the space of finite subsets of the metric space $X$ by $X^{*}$, then $\hat{m}$ takes values in the metric space

$$
\left([0,1] \times \mathbb{X} \times([0,1] \times \mathbb{X})^{*}\right)^{*}
$$


By conditioning on $\omega(\rho)=1$, we obtain a unimodular random rooted marked graph $(H, \rho, \hat{m})$. Since $(H, \rho)$ is strongly sofic, there exists a sequence of finite unimodular random rooted marked graphs $\left(H_{n}, \rho_{n}, \hat{m}_{n}\right)$ converging in distribution to $(H, \rho, \hat{m})$, and we may assume that $\mathbb{E}\left|\hat{m}_{n}\left(\rho_{n}\right)\right|<\infty$ for each $n \geq 1$. For each $\varepsilon>0$ and $R \in \mathbb{N}$, bias $\left(H_{n}, \rho_{n}, \hat{m}_{n}\right)$ by $|\hat{m}(\rho)|$ and construct a finite unimodular random rooted marked graph $\left(G_{n}^{R, \varepsilon}, \rho_{n}, m_{n}^{R, \varepsilon}\right)$ from $\left(H_{n}, \rho_{n}^{\prime}, \hat{m}_{n}\right)$ as follows.

(1) Let the vertex set of $G_{n}^{R, \varepsilon}$ be the union $\bigcup_{v \in H_{n}} \hat{m}(v)$, and let $\rho_{n}$ be chosen uniformly from $\hat{m}\left(\rho_{n}^{\prime}\right)$. For each vertex $u$ of $G_{n}^{R, \varepsilon}$, let $u=\left(u^{1}, u^{2}, u^{3}\right)$ be the three coordinates of $u$, and let $m(u)=u^{2}$.

(2) Draw an edge between two vertices $u_{1}$ and $u_{2}$ of $G_{n}^{R, \varepsilon}$ if and only if

(a) $u_{1} \in \hat{m}_{n}\left(v_{1}\right)$ and $u_{2} \in \hat{m}_{n}\left(v_{2}\right)$ for some vertices $v_{1}$ and $v_{2}$ of $H_{n}$ that are at distance at most $R$ in $H_{n}$, and

(b) there exists a pair of points

$$
(t, x) \in u^{3} \subset[0,1] \times \mathbb{X} \text { and }(s, y) \in u^{3} \subset[0,1] \times \mathbb{X}
$$

such that the distance between $(t, x)$ and $(s, y)$ is less than $\varepsilon$ in the product metric. If there are multiple such pairs, we draw multiple edges as appropriate.

(3) Let $\left\{Z_{n}(e)\right\}$ be a collection of i.i.d. Bernoulli-1/2 random variables indexed by the edges of $G_{n}^{R, \varepsilon}$. For each edge $e$ of $G_{n}^{R, \varepsilon}$, let $(t, x)$ and $(s, y)$ be the matching pair of points in $[0,1] \times \mathbb{X}$ that led us to draw $e$ in step $(2)$, and let $m_{n}^{R, \varepsilon}(e)=x$ if $Z_{n}(e)=0$ and $m_{n}^{R, \varepsilon}(e)=y$ if $Z_{n}(e)=1$.

This construction is continuous for the local topology, and hence for each fixed $\varepsilon$ and $R$, the finite marked graphs $\left(G_{n}^{R, \varepsilon}, \rho_{n}, m_{n}^{R, \varepsilon}\right)$ converge to the marked graph $\left(G^{R, \varepsilon}, \rho, m^{R, \varepsilon}\right)$ defined by applying the same procedure to $\left(H, \rho^{\prime}, \hat{m}\right)$. Taking $\epsilon \rightarrow 0$, we obtain the marked graph $\left(G^{R}, \rho, m^{R}\right)$ which consists of those edges of $(G, \rho)$ whose endpoints are of distance at most $R$ in $\omega$. Finally, taking $R \rightarrow \infty$ we recover $(G, \rho, m)$. Thus, $(G, \rho, m)$ is a weak limit of sofic unimodular random rooted marked graphs, and it follows that $(G, \rho, m)$ is sofic.

Proof of Theorem 2. Let $(M, \rho)$ be a simply connected unimodular random rooted map, let $(G, \rho)$ be the underlying graph of $M$, and let $\mathfrak{F}$ be a sample of $\mathrm{FUSF}_{M}$. By Theorem $5.13, \mathfrak{F}$ is connected almost surely, and so $(G, \rho)$ is coupling equivalent to the unimodular random rooted tree $(\mathfrak{F}, \rho)$. It follows from Theorems 8.1 and 8.2 that $(G, \rho)$ is strongly sofic. By encoding the map $(M, \rho)$ as a marking of $(G, \rho)$ as in Section 2.2 and [2, Example 9.6], we conclude that the unimodular random rooted map $(M, \rho)$ is also sofic.

\section{Open Problems}

We expect that the dichotomy of Theorem 1 extends to many further properties of planar unimodular random rooted maps. In this section, we discuss several such properties that might be addressed.

\subsection{Rates of escape of the random walk}

Can the type of a unimodular random planar map be determined by the rate of escape of the random walk? The work of Ding, Lee, and Peres [30] (together with the characterization of parabolic unimodular random planar maps as Benjamini-Schramm limits of finite planar 
maps) implies that the random walk is at most diffusive on any parabolic unimodular random planar map of finite expected degree.

Theorem 9.1 ([30]). There exists a universal constant $C$ such that for every parabolic unimodular random rooted map $(M, \rho)$ with $\mathbb{E}[\operatorname{deg}(\rho)]<\infty$, we have

$$
\mathbb{E}\left[\operatorname{deg}(\rho) d\left(\rho, X_{n}\right)^{2}\right] \leq C n \mathbb{E}[\operatorname{deg}(\rho)]
$$

for all $n \geq 0$.

On the other hand, if $(M, \rho)$ is a hyperbolic unimodular random rooted map with $\mathbb{E}[\operatorname{deg}(\rho)]<\infty$ that has at most exponential growth, meaning that

$$
\limsup _{n \rightarrow \infty} \frac{1}{n} \log |B(\rho, n)|<\infty,
$$

then the random walk on $M$ has positive speed, that is,

$$
\lim _{n \rightarrow \infty} \frac{1}{n} d\left(\rho, X_{n}\right)>0
$$

a.s., where the limit exists a.s. by Kingman's subadditive ergodic theorem. (Note that the exponential growth condition always holds for graphs of bounded degree.) This can be seen in several ways: it is an easy consequence of a theorem of Benjamini, Lyons, and Schramm [14, Theorem 3.2] (see also [2, Theorem 8.13] and [4, Theorem 3.2]) that every invariantly nonamenable unimodular random rooted graph with finite expected degree and at most exponential growth has positive speed. Meanwhile, it is a result of Benjamini and Curien [9], generalizing the work of Kaimanovich, Vershik, and others [54, 53, 52, 51, 50, 49], that every non-Liouville unimodular random rooted graph with finite expected degree and at most exponential growth has positive speed.

In general, however, there do exist invariantly nonamenable, non-Liouville, unimodular random rooted graphs with finite expected degree such that the random walk has zero speed almost surely. An example of such a graph will appear in a forthcoming paper by the second author. We do not know of a planar example, which motivates the following question.

Question 9.2. Let $(M, \rho)$ be a hyperbolic unimodular random rooted planar map with $\mathbb{E}[\operatorname{deg}(\rho)]<\infty$. Does the random walk on $M$ have positive speed almost surely?

See [4] for a related result concerning the positivity of the speed in the hyperbolic metric induced by the circle packing of a hyperbolic unimodular random rooted triangulation with $\mathbb{E}\left[\operatorname{deg}(\rho)^{2}\right]<\infty$.

\subsection{Positive harmonic functions}

Theorem 1 states that the existence of non-constant bounded harmonic functions and of non-constant harmonic Dirichlet functions are both determined by the type. We conjecture that a similar result holds for positive harmonic functions.

Conjecture 9.3. Let $(M, \rho)$ be a parabolic unimodular random rooted map and suppose that $\mathbb{E}[\operatorname{deg}(\rho)]<\infty$. Then $M$ does not admit any non-constant positive harmonic functions almost surely.

This conjecture would follow from a positive answer to the following question. 
Question 9.4. Let $(M, \rho)$ be a parabolic unimodular random rooted map and suppose that $\mathbb{E}[\operatorname{deg}(\rho)]<\infty$. Let $\left\langle X_{n}\right\rangle_{n \geq 0}$ be a simple random walk on $M$. Is every component of the complement of the trace of $\left\langle X_{n}\right\rangle_{n \geq 0}$ finite almost surely?

Note that the answer to Question 9.4 is trivially positive if $M$ is recurrent.

\section{Acknowledgment}

OA was supported by NSERC and the Simons Foundation. TH was supported by a Microsoft Research PhD Fellowship. AN was supported by ISF grant 1207/15, and ERC starting grant 676970 RANDGEOM. GR was supported in part by EPSRC grant EP/I03372X/1. Part of this work was conducted at the Isaac Newton Institute in Cambridge, during the programme 'Random Geometry' supported by EPSRC Grant Number EP/K032208/1.

\section{References}

[1] M. Aizenman, H. Kesten, and C. M. Newman. Uniqueness of the infinite cluster and continuity of connectivity functions for short and long range percolation. Comm. Math. Phys., 111(4):505-531, 1987.

[2] D. Aldous and R. Lyons. Processes on unimodular random networks. Electron. J. Probab., 12:no. 54, 1454-1508, 2007.

[3] A. D. Aleksandrov and V. A. Zalgaller. Intrinsic geometry of surfaces. Translated from the Russian by J. M. Danskin. Translations of Mathematical Monographs, Vol. 15. American Mathematical Society, Providence, R.I., 1967.

[4] O. Angel, T. Hutchcroft, A. Nachmias, and G. Ray. Unimodular hyperbolic triangulations: Circle packing and random walk. Inventiones Mathematicae, to appear, 2015. arXiv:1501.04677.

[5] O. Angel and G. Ray. Classification of half planar maps. Ann. Probab., to appear., 2013. arXiv:1303.6582.

[6] O. Angel and G. Ray. The half plane uipt is recurrent. arXiv preprint arXiv:1601.00410, 2016.

[7] O. Angel and O. Schramm. Uniform infinite planar triangulations. Comm. Math. Phys., 241(2-3):191-213, 2003.

[8] A. F. Beardon and K. Stephenson. Circle packings in different geometries. Tohoku Mathematical Journal, Second Series, 43(1):27-36, 1991.

[9] I. Benjamini and N. Curien. Ergodic theory on stationary random graphs. Electron. J. Probab., 17:no. 93, 20, 2012.

[10] I. Benjamini, N. Curien, and A. Georgakopoulos. The Liouville and the intersection properties are equivalent for planar graphs. Electron. Commun. Probab., 17:no. 42, 5, 2012.

[11] I. Benjamini, R. Lyons, Y. Peres, and O. Schramm. Critical percolation on any nonamenable group has no infinite clusters. Ann. Probab., 27(3):1347-1356, 1999.

[12] I. Benjamini, R. Lyons, Y. Peres, and O. Schramm. Group-invariant percolation on graphs. Geom. Funct. Anal., 9(1):29-66, 1999.

[13] I. Benjamini, R. Lyons, Y. Peres, and O. Schramm. Uniform spanning forests. Ann. Probab., 29(1):1-65, 2001.

[14] I. Benjamini, R. Lyons, and O. Schramm. Percolation perturbations in potential theory and random walks. In Random walks and discrete potential theory (Cortona, 1997), Sympos. Math., XXXIX, pages 56-84. Cambridge Univ. Press, Cambridge, 1999.

[15] I. Benjamini, R. Lyons, and O. Schramm. Unimodular random trees. arXiv:1207.1752, 2012.

[16] I. Benjamini, E. Paquette, and J. Pfeffer. Anchored expansion, speed, and the hyperbolic Poisson Voronoi tessellation. arXiv:1409.4312.

[17] I. Benjamini and O. Schramm. Harmonic functions on planar and almost planar graphs and manifolds, via circle packings. Invent. Math., 126(3):565-587, 1996.

[18] I. Benjamini and O. Schramm. Random walks and harmonic functions on infinite planar graphs using square tilings. Ann. Probab., 24(3):1219-1238, 1996.

[19] I. Benjamini and O. Schramm. Percolation in the hyperbolic plane, 2000. 
[20] I. Benjamini and O. Schramm. Recurrence of distributional limits of finite planar graphs. Electron. J. Probab., 6:no. 23, 1-13, 2001.

[21] I. Benjamini and O. Schramm. Percolation beyond $\mathbb{Z}^{d}$, many questions and a few answers [mr1423907]. In Selected works of Oded Schramm. Volume 1, 2, Sel. Works Probab. Stat., pages 679-690. Springer, New York, 2011.

[22] I. Biringer and J. Raimbault. The topology of invariant random surfaces. http://arxiv.org/abs/1411.0561.

[23] L. Bowen. Periodicity and circle packings of the hyperbolic plane. Geom. Dedicata, 102:213-236, 2003.

[24] L. Brouwer. On the structure of perfect sets of points. In KNAW, Proceedings, volume 12, pages 1909-1910.

[25] R. M. Burton and M. Keane. Density and uniqueness in percolation. Communications in mathematical physics, 121(3):501-505, 1989.

[26] B. Chen. The gauss-bonnet formula of polytopal manifolds and the characterization of embedded graphs with nonnegative curvature. Proceedings of the American Mathematical Society, 137(5):1601-1611, 2009.

[27] N. Curien. A glimpse of the conformal structure of random planar maps. arXiv:1308.1807, 2013.

[28] N. Curien. Planar stochastic hyperbolic infinite triangulations. arXiv:1401.3297, 2014.

[29] N. Curien, L. Ménard, and G. Miermont. A view from infinity of the uniform infinite planar quadrangulation. ALEA Lat. Am. J. Probab. Math. Stat., 10(1):45-88, 2013.

[30] J. Ding, J. R. Lee, and Y. Peres. Markov type and threshold embeddings. Geometric and Functional Analysis, 23(4):1207-1229, 2013.

[31] R. J. Duffin. The extremal length of a network. J. Math. Anal. Appl., 5:200-215, 1962.

[32] G. Elek. On the limit of large girth graph sequences. Combinatorica, 30(5):553-563, 2010.

[33] G. Elek and G. Lippner. Sofic equivalence relations. J. Funct. Anal., 258(5):1692-1708, 2010.

[34] A. Gandolfi, M. Keane, and C. Newman. Uniqueness of the infinite component in a random graph with applications to percolation and spin glasses. Probability Theory and Related Fields, 92(4):511-527, 1992.

[35] C. Garban. Quantum gravity and the KPZ formula [after Duplantier-Sheffield]. Astérisque, (352):Exp. No. 1052, ix, 315-354, 2013. Séminaire Bourbaki. Vol. 2011/2012. Exposés 1043-1058.

[36] J. R. Gilbert, J. P. Hutchinson, and R. E. Tarjan. A separator theorem for graphs of bounded genus. Journal of Algorithms, 5(3):391-407, 1984.

[37] J. T. Gill and S. Rohde. On the Riemann surface type of random planar maps. Rev. Mat. Iberoam., 29(3):1071-1090, 2013.

[38] O. Gurel-Gurevich and A. Nachmias. Recurrence of planar graph limits. Ann. of Math. (2), 177(2):761781, 2013.

[39] O. Gurel-Gurevich and A. Nachmias. Recurrence of planar graph limits. Ann. of Math. (2), 177(2):761781, 2013.

[40] O. Häggström. Infinite clusters in dependent automorphism invariant percolation on trees. Ann. Probab., 25(3):1423-1436, 1997.

[41] O. Häggström and Y. Peres. Monotonicity of uniqueness for percolation on Cayley graphs: all infinite clusters are born simultaneously. Probab. Theory Related Fields, 113(2):273-285, 1999.

[42] A. Hatcher. Algebraic topology. Cambridge University Press, Cambridge, 2002.

[43] Z.-X. He and O. Schramm. Fixed points, Koebe uniformization and circle packings. Ann. of Math. (2), 137(2):369-406, 1993.

[44] Z.-X. He and O. Schramm. Hyperbolic and parabolic packings. Discrete Comput. Geom., 14(2):123-149, 1995.

[45] Y. Higuchi. Combinatorial curvature for planar graphs. Journal of Graph Theory, 38(4):220-229, 2001.

[46] T. Hutchcroft. Interlacements and the wired uniform spanning forest. arXiv:1512.08509, 2015.

[47] T. Hutchcroft. Wired cycle-breaking dynamics for uniform spanning forests. arXiv:1504.03928, 2015.

[48] T. Hutchcroft and A. Nachmias. Uniform spanning forests of planar graphs. http://arxiv.org/abs/1603.07320.

[49] V. Kaimanovich. Boundary and entropy of random walks in random environment. Prob. Theory and Math. Stat, 1:573-579, 1990.

[50] V. A. Kaimanovich. Hausdorff dimension of the harmonic measure on trees. Ergodic Theory and Dynamical Systems, 18(03):631-660, 1998.

[51] V. A. Kaimanovich. Random walks on sierpiński graphs: hyperbolicity and stochastic homogenization. In Fractals in Graz 2001, pages 145-183. Springer, 2003. 
[52] V. A. Kaimanovich, Y. Kifer, and B.-Z. Rubshtein. Boundaries and harmonic functions for random walks with random transition probabilities. Journal of Theoretical Probability, 17(3):605-646, 2004.

[53] V. A. Kaimanovich and A. M. Vershik. Random walks on discrete groups: boundary and entropy. The annals of probability, pages 457-490, 1983.

[54] V. A. Kaimanovich and W. Woess. Boundary and entropy of space homogeneous markov chains. Annals of probability, pages 323-363, 2002.

[55] M. Krikun. Local structure of random quadrangulations. arXiv:math/0512304, 2005.

[56] S. K. Lando and A. K. Zvonkin. Graphs on surfaces and their applications, volume 141 of Encyclopaedia of Mathematical Sciences. Springer-Verlag, Berlin, 2004. With an appendix by Don B. Zagier, LowDimensional Topology, II.

[57] G. F. Lawler. A self-avoiding random walk. Duke Math. J., 47(3):655-693, 1980.

[58] R. J. Lipton and R. E. Tarjan. Applications of a planar separator theorem. SIAM J. Comput., 9(3):615627,1980 .

[59] R. Lyons, B. J. Morris, and O. Schramm. Ends in uniform spanning forests. Electron. J. Probab., 13:no. $58,1702-1725,2008$.

[60] R. Lyons and Y. Peres. Probability on Trees and Networks. Cambridge University Press, 2015. In preparation. Current version available at http://mypage.iu.edu/ ${ }^{\text {rdlyons/. }}$

[61] R. Lyons, Y. Peres, and O. Schramm. Minimal spanning forests. Ann. Probab., 34(5):1665-1692, 2006.

[62] R. Lyons and O. Schramm. Indistinguishability of percolation clusters. Ann. Probab., 27(4):1809-1836, 1999.

[63] C. M. Newman and L. S. Schulman. Infinite clusters in percolation models. J. Statist. Phys., 26(3):613-628, 1981.

[64] R. Pemantle. Choosing a spanning tree for the integer lattice uniformly. Ann. Probab., 19(4):1559-1574, 1991.

[65] I. Richards. On the classification of noncompact surfaces. Transactions of the American Mathematical Society, 106(2):259-269, 1963.

[66] K. Stephenson. Introduction to circle packing. Cambridge University Press, Cambridge, 2005. The theory of discrete analytic functions.

[67] D. B. Wilson. Generating random spanning trees more quickly than the cover time. In Proceedings of the Twenty-eighth Annual ACM Symposium on the Theory of Computing (Philadelphia, PA, 1996), pages 296-303. ACM, New York, 1996.

OMER ANGel

Department of Mathematics, University of British Columbia

Email: angel@math.ubc.ca

TOM HutchCROFT

Statistical Laboratory, DPMMS, University of CAmbridge

Email: t.hutchcroft@maths.cam.ac.uk

Asaf NaChMias

School of Mathematical Sciences, Tel Aviv University

Email: asafnach@post.tau.ac.il

Gourab Ray

Department of Mathematics and Statistics, University of Victoria

Email: gourab1987@gmail.com 